Review

\title{
Human Gut Microbiota: Friend or Foe?
}

Monika Pliszka, Leszek Szablewski*

Medical University of Warsaw, Chair and Department of General Biology and Parasitology, 5 Chalubinskiego Str, 02-004 Warsaw, Poland; E-Mail: monika.pliszka@wum.edu.pl; leszek.szablewski@wum.edu.pl

* Correspondence: Leszek Szablewski; E-Mail: leszek.szablewski@wum.edu.pl

Academic Editor: Tatsuo Kanda

OBM Hepatology and Gastroenterology

2020 , volume 4 , issue 3

doi: 10.21926/obm.hg.2003050
Received: March 06, 2020

Accepted: July 14, 2020

Published: August 07, 2020

\begin{abstract}
The human gut is a house to approximately 1,000 different species of bacteria. The bacterial composition of gut microbiota is influenced by several factors, including age, sex, mode of delivery, geographical location, ethnicity, diet, drugs, and administration of prebiotics and/or probiotics. Similarly, human health depends on the composition of gut microbiome, with gut bacteria playing a crucial role in human physiology. For instance, gut microbiota synthesizes vitamins and amino acids, and affects the biotransformation of bile acid. Intestinal microbiota produces short-chain fatty acids (SCFAs) that stimulate intestinal gluconeogenesis, protect the host from diet-induced obesity, and may play a role as an energy substrate. Changes in the composition of gut microbiota, termed dysbiosis, due to, for example, change in the diet or uptake of certain drugs, may result in metabolic diseases, autoimmune and allergic diseases, cancers, and many others. Conversely, dysbiosis can be a consequence of a disease in several cases. This review outlines the current knowledge of the associations between human gut microbiota and human health and diseases.
\end{abstract}

\section{Keywords}

Gut microbiota; physiology; pathology

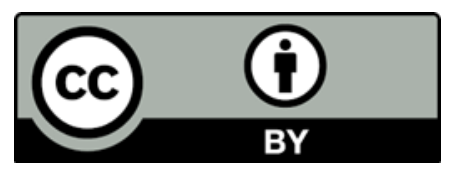

(C) 2020 by the author. This is an open access article distributed under the conditions of the Creative Commons by Attribution License, which permits unrestricted use, distribution, and reproduction in any medium or format, provided the original work is correctly cited. 


\section{Abbreviations}

APJ - Apelin receptor; BCAAs - Branched-chain amino acids; BCFAs - Branched-chain fatty acids; $\mathrm{CB}_{1} \mathrm{R}, \mathrm{CB}_{2} \mathrm{R}$ - Cannobinoid receptors 1 and 2; eCB - Endocannabinoid system; FMT - Faecal microbial transplantation; FXR - Farnesoid X-activated receptor; GLP-1, GLP-2 - Glucagon-like peptide 1 and 2; GPBAR1 - G-protein-coupled bile acid receptor 1; HFD - High-fat diet; IL - Interleukin; IMT Intestinal microflora transplantation; LPS - Lipopolysaccharide; MAPK - Mitogen-activated protein kinase; NF-кB - Nuclear factor-kB; PYY - Peptide YY, peptide tyrosine-tyrosine; SCFAs - Short-chain fatty acids; SFAs - Saturated fatty acids; TLRs - Toll-like receptors; TNF- $\alpha$ - Tumor necrosis factor- $\alpha$

\section{Introduction}

The adult intestine contains an average of $10^{14}$ microorganisms $/ \mathrm{mL}$ of luminal content and weighs around $1.5 \mathrm{~kg}$. The majority of the gut microbiota resides in the colon [1, 2]. The density of bacteria changes in the gastrointestinal tract from $10^{2}$ to $10^{4}$ cells $/ \mathrm{mL}$ in the stomach to more than $10^{12}$ cells $/ \mathrm{mL}$ in the colon [3]. The majority of gut microbiota consists of bacteria and Archaea, with a small number of viruses, fungi, and protists (protozoa).

Approximately 1,000 different species of bacteria reside in the gut. The most abundant phyla are: 1) Firmicutes; these are gram-positive bacteria, which account for 60 to $80 \%$ of gut microbiota and consists of more than 200 genera, most important of which are Ruminococcus, Mycoplasma, Clostridium, and Lactobacillus; 2) Bacteroidetes (20-30\%); these are gram-negative bacteria and include the following genera: Bacteroides, Prevotella, Xylanibacter; 3$)$ Actinobacteria ( $<10 \%)$; these are gram-positive bacteria and include the genus Bifidobacterium; 4) Proteobacteria ( $<1 \%)$, these are gram-negative bacteria with genus Escherichia and family Enterobacteriaceae [1, 4].

The bacterial composition of gut microbiota is influenced by several factors, such as age, sex, geographical location, ethnicity, diet, and drugs [5]. The gastrointestinal tract of the newborn is sterile. The composition of the gut microbiota in infants depends on the mode of delivery. In the case of vaginal delivery, newborns develop microbiota within $20 \mathrm{~min}$ of birth from the maternal vagina or fecal microflora (Lactobacillus, Prevotella, and Sneathia). Babies born by cesarean section have a gut with bacteria resembling those from mother's skin and hands that touch the newborn after birth (Staphylococcus, Corynebacterium, and Propionibacterium) [6]. In the few days after delivery, the infant's microbiota primarily comprises Proteobacteria and Actinobacteria [1, 7]. The composition of gut microbiota changes during life, and as mentioned above, depends on several factors. In infants, the gut microbiota is narrow and unstable. At the end of the first year of life, the gut microbiota changes to a state to resemble that of an adult. At approximately 2.5 years of age, the bacterial composition is like that of the adult microbiota. From this stage, Firmicutes and Bacteroidetes start predominating [1], such that from the age of seven years, Firmicutes and Bacteroidetes comprise approximately $90 \%$ of the bacterial composition of the gut. The remaining $10 \%$ of microorganisms include Tericutes, Cyanobacteria, and Proteobacteria. From this stage, the gut bacteria become more stable and are characteristic of that present in the adults [6]. During adulthood, the composition of the microbiota is stable; however, it depends on several factors. According to Wu et al. [8], three enterotypes are present in the adult gut: Bacteroides, Prevotella, and Ruminococcus.

In this review, we focus on the role of gut microbiota in maintaining human health, metabolism, and homeostasis. Moreover, the gut microbiota protects against invading pathogens. In contrast, 
alterations in the composition of gut microbiota, termed dysbiosis, may result in the development of multiple diseases, such as autoimmune diseases, metabolic diseases, neurodevelopmental disorders, neurodegenerative disorders, as well as progression and development of cancers.

\section{Role of Gut Microbiota in Human Health}

The gut microbiota influences human health by using the metabolic pathways to produce numerous small molecules. Some of these molecules are absorbed into the circulating system. These can be chemically modified by the host and secreted in the urine [9]. For example, the gut microbiota synthesizes vitamins and amino acids and influences the biotransformation of bile acids [10]. Further, the intestinal microbiota produces short-chain fatty acids (SCFAs) (butyrate, acetate, and propionate) in the cecum and colon [11], which are absorbed into the circulatory system [11]. 21The increased levels of propionate promote intestinal gluconeogenesis [12], which are associated with the microbiota following gastric bypass [13]. It protects from diet-induced obesity [14] by transducing signals through the central nervous system and resulting in glucose intolerance [12]. Butyrate, another SCFA produced by gut microbiota, acts as an energy substrate for colonocytes [15, $16]$ and reduces energy availability [17]. Similarly, acetate acts as a histone deacetylase inhibitor [18-20].

The gut microbiota produces other metabolites, such as branched-chain fatty acids, amines, alcohols (mainly yielded from monosaccharides); ammonia, sulfur compounds, phenols, and indoles (derived from amino acids); glycerol and choline derivatives (obtained from the breakdown of lipids); and tertiary cycling of carbon dioxide and hydrogen [21]. Branched-chain fatty acids (BCFAs), such as isovalerate and isobutyrate, generated from valine and leucine fermentation, respectively, are often used as biomarkers of protein catabolism. BCFAs contribute to the production of $5 \%$ of total SCFAs [22].

However, little is known about the effect of BCFAs on human health. It is reported that similar to SCFAs, BCFAs can modulate glucose and lipid metabolism in the liver. For instance, isobutyric and isovaleric acids are involved in human lipid metabolism. These metabolites are rare in internal human tissues; however, they are present in high concentrations in the skin and vernix caseosa, the unique waxy white substance coating the skin of term newborns, and in the healthy term infant's gut. A study involving a mouse model of the tumor and cultured cells reported that BCFAs inhibited the tumor growth, leading to the speculation that these could induce apoptosis in human breast cancer cells [21].

Primary bile acids, such as cholic acid and chenodeoxycholic acid, are synthesized from cholesterol by the host in hepatocytes [20]. These acids are metabolized by microbiota to generate secondary bile acids [23, 24]: deoxycholic acid (DCA), and lithocholic acid (LCA). Bile acids act not only as naturally occurring detergents that help to absorb dietary fats but also function as signaling molecules. Bile acids bind to their specific receptors: G-protein-coupled bile acid receptor 1 (GPBAR1, also known as TGR5) and the farnesoid X-activated receptor (FXR, also known as the bile acid receptor) [25] to affect host metabolism. Furthermore, gut microbiota produces agonists that regulate TGR5 signaling [26]. They also regulate FXR signaling by reducing the levels of tauro- $\beta$ muricholic acid, a naturally occurring antagonist [27]. Secondary bile acids act as ligands and bind to TGR5 and G-protein coupled receptors (GPCRs) and stimulate the production of GLP-1 in L-cells. The secreted peptide increases energy expenditure in muscles and improves insulin sensitivity [28]. 
The gut microbiota synthesizes and releases glycoside hydrolases that hydrolyze plant polysaccharides into monosaccharides and SCFAs. Short-chain fatty acids function as ligands for two receptors on endocrine cells in the gut: G-protein-coupled receptor 41 (GPR41) and G-proteincoupled receptor 43 (GPR43) [29]. These fatty acids bind to distinct receptors and stimulate the secretion of peptide YY (PYY, peptide tyrosine-tyrosine). The secreted PYY slows the intestinal transit by inhibiting the gut motility and thus enhances the absorption of nutrients in the intestine [10].

The gut microbiota plays a crucial role in the development of the immune system [30]. Metabolites secreted by microbiota act as signals for the development of regulatory T-cells, T-helper type 1 and type 2 cells, as well as T-helper 17 cells [31]. Short-chain fatty acids, especially butyrate, exert strong immunomodulatory effects. These metabolites stimulate the synthesis and release of protective peptides, cytokines, chemokines, and phagocytes [183] [488]. Moreover, they protect the commensal bacteria, initiate an inflammatory response to pathogenic organisms, and/or induce apoptosis [10]. The building of the mucus layer and secretion of mucins in the intestine by these bacteria may strengthen the intestinal barrier. Moreover, it has been proposed that the gut microbiota affects the development of different types of cells and tissues [32].

Short-chain fatty acids produced during the bacterial fermentation of insoluble dietary fibers in the intestine bind to GPR41 and GPR43 [33]. This binding reduces the inflammation in immune cells and stimulates $L$ cells to release glucagon-like peptide 1 (GLP-1) and PYY. These peptides improve the insulin sensitivity of cells and tissues [34]. Butyrate, an SCFA, acts as an energy substrate in the mucosal cells of the colon, whereas acetate and propionate enhance hepatic gluconeogenesis and lipogenesis [28].

Several other metabolic products of bacteria are known to be associated with human health and diseases. For example, methylamines (trimethylamine, trimethylamine- $\mathrm{N}$-oxide, etc.) are linked with diseases, such as progressive renal fibrosis/dysfunction, insulin resistance, non-alcoholic fatty liver disease, and atherosclerosis. Branched-chain amino acids (BCAAs), membrane phospholipids, and triacylglycerols, are associated with insulin resistance, whereas aromatic amino acids (AAAs), best-known for their role in the nervous system, influence human health. Further, phenylacetic acid, a microbial product of phenylalanine, is associated with hepatic steatosis and increased lipid storage in human primary hepatocytes and in animal models [35].

\section{Modulators of Gut Microbiota}

Several factors are known to influence the composition of gut microbiota. It is recently accepted that changes in the gut microbiota, termed dysbiosis, can have negative consequences because of the changes in the metabolic activity of gut bacteria. For example, it can disturb the metabolism and result in several diseases such as autoimmune and allergic diseases [36-38], metabolic diseases $[39,40]$, and several others.

\subsection{Diet}

Diet is one of the important factors that can change the composition of microbiota. The traditional diets of most pre-agricultural people were low in carbohydrates [41, 42]. However, with industrialization, the intake of fat and saturated fatty acids has increased rapidly. In contrast, the composition of dietary fiber and diet with a low glycemic index decreased [43]. Human studies 
demonstrated that switching from a fat-rich to low fiber diet changes the gut microbiota in only 24 h [1]. Dietary fat decreases the population of Lactobacillus and increases that of gram-negative bacteria [44, 45]. For example, De Filippo et al. [46] found gut microbiota in European children to be depleted of Bacteroides and enriched in Enterobacteriaceae as compared to the gut microbiota in rural African children. The diet of the African children from an African village Burkina Faso, is mostly derived from plants. Therefore, they have greater proportions of Bacteroidetes and grampositive bacteria. These children have increased Bacteroidetes: Firmicutes ratio in addition to an abundance of Prevotella and Xylanibacter. The genus Xylanibacter was found to be absent in European children. European children have a low intake of dietary fibers and a high intake of animal proteins and saturated fats. This "western diet" and lifestyle promote the growth of Firmicutes and gram-negative bacteria [46]. Moreover, these findings were confirmed in animal studies [47]. The carbohydrate-reduced and calorie-restricted diets also influence the composition of gut microbiota $[48,49]$. For example, mice consuming the "western diet" had a significantly lower level of bacterial diversity, indicating unhealthy gut microbiota [50]. Claesson et al. [51] reported that decreased diversity in the gut microbiota is linked to increased frailty and poor health [51]. These mice had a significantly higher proportion of Firmicutes and a lower proportion of Bacteroidetes as compared to mice on a low-fat diet rich in complex plant polysaccharides [52].

The type of diet exerts a strong effect on the composition of microbiota. For example, the Mediterranean diet, which predominantly consists of fruits, vegetables, grains, and monounsaturated fats, is suggested as the gold standard for optimum health [53]. This diet can change the ratio of Bacteroidetes and Clostridium (increases): Proteobacteria and Bacillaceae (decreases) [54]. Italian people that prefer the Mediterranean diet have a higher abundance of Prevotella and SCFAs [55]. The vegetarian and vegan diets in healthy subjects decreases Bacteroides fragilis as compared to that in subjects on an omnivore diet [56]. These results have been described in detail by Matijàši et al. [57]. Researchers observed that in comparison to an omnivore diet, vegetarian diet in Slovenian people, increased the ratio of Bacteroides-Prevotella, levels of Bacteroides thetaiotaomicron, Clostridium clostridioforme, and Faecalibacterium prausnitzii but decreased the count of Clostridium cluster XIVa [57]. High-fibered diet, rich in plant fibers, promote the diversification of the microbiota $[46,58,59]$. This diet significantly increases the microbial counts of Actinobacteria and Bacteroidetes and increases the Firmicutes: Bacteroidetes ratio [8], and increases the number of bifidobacteria, which is a positive indicator of prebiotic activity [60].

High-fat diet, as mentioned earlier, decreases the number of Bacteroidetes and increases that of Firmicutes [60]. The characteristic "high-fat, western diet" results in an elevated microbial count of Bacteroides enterotype (Alistipes, Bilophila, and Bacteroides) [8] and decreased levels of Firmicutes that metabolize plant polysaccharides (Roseburia, Eubacterium rectale, Ruminococcus bromii) [60, 61].

The following points summarize the influence of diet on the composition of gut microbiota [59, 62]; $\uparrow$ refers to increase, $\downarrow$ refers to decrease:

1) Rich in plant-derived polysaccharides: Actinobacteria, Bacteroidetes $\uparrow$, Firmicutes $\downarrow$, diversification of the microbiota $\uparrow$;

2) Vegetarian diet: ratio Bacteroides-Prevotella, B. thetaiotaomicron, C. clostridioforme, F. prausnitzii $\uparrow$, Clostridium cluster XIVa $\downarrow$;

3) Mediterranean diet: Bacteroidetes, Clostridium, Prevotella $\uparrow$, Bacillaceae, Proteobacteria $\downarrow$; 
4) High-fat diet: Firmicutes $\downarrow(\uparrow)$, Bacteroidetes $\uparrow(\downarrow)$; varying results have been obtained in different studies. Observed differences may be due to different methods used in a particular study, use of different organisms (human and animal models), as well as use of different foods, such as fat, during experiments.

5) High-protein diet: Bacteroides enterotype $\uparrow$, Firmicutes, Ruminococcus bromii $\downarrow$;

6) Reduced variety due to long-stay care: Bacteroidetes $\uparrow$, overall diversity $\downarrow$;

7) Change from a vegetarian diet to "western" diet: Bacteroides $\uparrow$, Prevotella $\downarrow$;

8) Calorie-restricted diet: ratio Firmicutes to Bacteroidetes $\downarrow$.

\subsection{Effect of Drugs on the Composition of Gut Microbiota}

The increased use of antibiotics greatly affects the composition of gut microbiota. As reported by Jernberg et al. [63] and Dethlefsen and Relman [64], certain taxons could not be recovered even several months after the use of antibiotics. The use of antibiotics in the very early stage of infancy could disturb the healthy microbiota with adverse metabolic consequences[5]. A decrease in bifidobacteria and Bacteroides, which are antiobesogenic bacteria was observed. The use of antibiotics slowed the regrowth of bifidobacteria and prevented the re-establishment of Bacteroides spp. [65]. The effect of antibiotics on children and adults is associated with reduced diversity of gut microbiota [66]. It was found that a 5-day antibiotic treatment changed the human composition of gut microbiota for up to four weeks. Following this, the composition of gut microbiota reverted to the composition present before the administration of antibiotics. However, certain communities failed to recover within six months [67]. The antibiotic therapy may cause antibiotic-associated diarrhea due to the pathological overgrowth of Clostridium difficile [68]. Antibiotic therapy in neonates increases the risk of intestinal intussusception [69]. The use of antibiotics in critically ill patients reduces gut microbial diversity due to the overgrowth of Enterococci [70].

Metformin is widely used in patients with type 2 diabetes. Its hypoglycemic effect is attributed to the suppressed glucose output from the liver through the activation of AMPK-dependent and AMPK-independent pathways. Further, metformin might modify the gut microbiota [71]. Significant alterations in 81 and 86 strains of gut microbiota, respectively, were reported in patients with type 2 diabetes treated for two and four months with metformin. The majority of these belonged to $\mathrm{Y}$ proteobacteria (for example E. coli) and Firmicutes [72]. Moreover, researchers observed increased number of Bifidobacterium and E. coli and decreased number of Intestinibacter in patients treated with metformin. Similarly, abundant Akkermansia muciniphila were observed in patients after four months of treatment with metformin. In vitro studies reported that metformin promoted the growth of Bifidobacterium adolescentis and $A$. muciniphila, but not E. coli.

\subsection{Role of Probiotics}

According to the World Health Organization (WHO), probiotics are "live microorganisms which, when administered in adequate amounts, confer a health benefit on the host" [73]. The currently used probiotics include majorly lactic acid bacteria, such as Lactobacillus (L. salivarius, L. paracasei, L. reuteri, L. plantarum, L. gaseri) and Bifidobacterium lactis [74, 75]. According to Rijkers et al. [76], probiotics influence the growth or survival of pathogenic microorganisms, thus improving the mucosal barrier function or mucosal immune system. Moreover, it affects the systemic immune 
system and other organs. In animal studies, the administration of certain strains of Lactobacillus had both an antidiabetic effect and reduced endotoxemia [77-79]. Obesity and diabetes in these animals were prevented by increasing the number of butyrate-producing bacteria. The administration of probiotics enhanced the secretion of GLP-1, a hunger-reducing hormone. Furthermore, the expression of genes involved in the synthesis and excretion of GLP-1 was upregulated [80].

\subsection{Role of Prebiotics}

According to Gibson and Roberfroid [81], "prebiotics" is defined as "a non-digestible food ingredient that positively affects the host by selectively stimulating the growth and activity of one or a limited number of bacteria in the colon, thus improving the host health." Prebiotics are selectively fermented by colonic bacteria; however, these are not hydrolyzed by human intestinal enzymes. Examples of prebiotics include inulin, oligosaccharides, and polyphenols in red wine. Animal studies showed that mice on a high-fat diet and fed prebiotics containing oligofructose showed restored levels of bifidobacteria, decreased endotoxemia, and improved glucose tolerance [82]. The administration of prebiotics increased the abundance of Bifidobacteriaceae and decreased the number of bacteria belonging to the Bacteroidaceae family [83]. Other studies reported that the administration of prebiotics reduced the levels of triglycerides, and intestinal permeability and inflammation [81, 84], reduced high-fat, diet-induced lipopolysaccharide endotoxemia, systemic inflammation, and liver inflammation [85]. Prebiotics stimulate the secretion of gut peptides: GLP-1 and peptide YY, which control the appetite sensation and glucose excursion responses following a meal in healthy subjects [86]. The effects of red wine consumption include increased levels of Enterococcus, Prevotella, Bifidobacterium, Bacteroides uniformis, Eggerthella lenta, Blautia coccoides, and reduced levels of lipopolysaccharide (LPS); these changes are attributed to the polyphenols present in red wine $[87,88]$.

\subsection{Role of Macrobiotic Ma-Pi 2 diet}

The Ma-2Pi 2 diet contains low amounts of fat (16-18\% of total energy), protein ( $12 \%$ of total energy), and fructose. The diet is rich in complex carbohydrates and comprises whole-grain cereals, vegetables, legumes, natural fibers, and other prebiotic products. It is devoid of animal protein and sugar $[75,89]$. In patients with type 2 diabetes, treated with macrobiotic Ma-Pi 2 diet, a positive effect on glycemia, $\mathrm{HbA}_{1}$, insulinemia, total cholesterol, low-density lipoprotein (LDL), high-density lipoprotein (HDL), and other biochemical parameters was observed [10]. Authors conclude that this diet affects the gut microbiota, because it contains large quantities of prebiotics and probiotics.

\subsection{Intestinal Microflora Transplantation}

Intestinal microflora transplantation (IMT) [84], also known as fecal microbial transplantation (FMT) [59], is a method of transplantation of fecal bacteria from a healthy "donor" to a recipient with disrupted or altered microbiota. This method is less accepted by patients in comparison to other methods, such as the administration of probiotics and/or prebiotics. This method is commonly used to treat recurrent $C$. difficile infections (CDIs). It uses endoscopy to introduce microbiota into the large intestine or into the duodenum, in the case of metabolic syndrome [84] . It was found that 
in patients with CDI who were deficient in both Bacteroidetes and Firmicutes, the gut microbiota changed to closely resemble the donor's microflora 14 days after bacteriotherapy (FMT) $[90,91]$.

\section{Metabolic Endotoxemia}

A high-fat diet (HFD), such as the western-style diet, is rich in saturated fatty acids (SFAs). This diet contains 35 to $45 \%$ of calories derived from fat, of which $11.1 \%$ is derived from SFAs alone [92]. Such a diet promotes the overgrowth of gram-negative bacteria [93]. Cani et al. [94] reported an association between the levels of serum LPS and the fed state, and that an HFD was responsible for three-fold greater serum LPS in mice. In addition, a study showed that HFD feeding significantly enriched the gut microflora with LPS-containing bacteria [94]. Lipopolysaccharide is a component of gram-negative bacteria [92]. It is absorbed by enterocytes, carried in plasma, and binds to chylomicrons [95]. It induces inflammation and metabolic diseases [94], and its concentration depends on the number of gram-negative bacteria. Metabolic endotoxemia is described as an increased level of LPS in the plasma [94, 96]. Toll-like receptors (TLRs) play a key role in innate immunity. Studies in animals demonstrated that LPS binds to the CD14/TLR4 receptor in macrophages (CD14/TLR4) [97] and triggers an inflammatory cascade [94, 98]. It was found that in healthy human subjects, endotoxemia increased the levels of adipose tumor necrosis factor- $\alpha$ (TNF$\alpha$ ) and interleukin-6 (IL-6), thus promoting insulin resistance. Moreover, researchers reported that the western diet increased the postprandial plasma LPS levels, correlated with increased mononuclear cell expression of TLR-4, nuclear factor-KB (NF-KB), and suppressed cytokine signaling-3 (SOCS-3), which is involved in insulin resistance. These changes were not observed after a diet rich in fibers and fruits $[99,100]$. Because LPS induces metabolic endotoxemia, it may be treated as a first step for the development of insulin resistance and diabetes [94, 101]. In humans, increased levels of circulating LPS stimulate the TLR-4/CD14 complex with subsequent stimulation of TLR-2-mediated inflammatory response and increased secretion of proinflammatory cytokines by the adipose tissue [102]. Moreover, another mechanism was reported [103]. Researchers suggested that changes in the composition of gut microbiota were attributed to HFD, characteristic of the western diet, and increased luminal LPS and gut permeability. Increased gut permeability increases the plasma levels of LPS, resulting in hyperphagia and obesity.

\section{The Endocannabinoid System and Apelinergic System}

According to Guerts et al. [104], two physiological systems are linked to the gut microbiota: the endocannabinoid system (eCB) and apelinergic system.

The endocannabinoid system acts as a link between gut microbiota, development of obesity, and related disorders such as type 2 diabetes [101]. This system is widely expressed in tissues and organs and controls the energy balance by regulating the feeding behavior and metabolism [105]. A close relation between LPS, metabolic endotoxemia, and eCB was shown [106]. The endocannabinoid system consists of bioactive lipids that activate specific $G$ protein-coupled receptors known as cannabinoid receptors 1 and $2\left(C B_{1} R\right.$ and $\left.C B_{2} R\right)$. The most studied lipids present in the body are $N$ arachidonoylethanolamine (anandamide, AEA) and 2-arachidonylglycerol (2-AG) [107]. The balance between the synthesis and inactivation of these lipids regulate their tissue levels [108]. Gut microbiota controls the eCB and regulates the expression of $C B_{1} R$ in the intestine and adipose tissue 
[101]. LPS controls the synthesis of eCB in macrophages, and macrophage infiltration depends on LPS activation.

Both eCB and LPS influence the adipose tissue. Physiological levels of LPS reduces differentiation and adipogenesis. In contrast, the activation of eCB has been reported to increase adipogenesis in an animal model. According to the suggestion proposed by Cani et al. [101], the eCB system, through $\mathrm{CB}_{1} \mathrm{R}$-dependent mechanisms, contributes to the gut barrier function and metabolic endotoxemia. This system controls the gut barrier function via distribution and localization of tight junction proteins (Zonula occludens-1 [zonulin] and occludin). Blockage of $\mathrm{CB}_{1} \mathrm{R}$ in obese mice lowered the alteration of zonulin and occludin and decreased the plasma levels of LPS [109]. The administration of eCB agonist increased the LPS levels and metabolic endotoxemia through changes in the permeability $[109,110]$. The eCB system is overactivated in obese humans and rodents.

The apelinergic system consists of apelin and apelin receptor (APJ). Apelin is an adipokine synthesized and secreted by adipocytes. Studies in animals demonstrated that intravenous intracerebroventricular injections of apelin lowered the glucose levels via eNOS, MAPK (mitogenactivated protein kinase), and Akt-dependent pathways, and restored glucose metabolism in obese and insulin-resistant mice $[111,112]$. The high doses of apelin, observed in obese/diabetic mice, caused pathologies such as hyperinsulinemia, hyperglycemia, glucose intolerance, and insulin resistance in normal mice that were put on fasting [112]. It was observed that under physiological conditions, eCB downregulates the expression of apelin and its receptors. In contrast, stimulation of eCB decreased the expression of apelin and APJ in adipose tissue, and LPS stimulated apelin and apelin receptors in adipose tissue [113]. Also other authors have found several positive and negative correlations between the gut microbiota and apelinergic system.

\section{Role of Dysbiosis in Development of Diseases}

The healthy gut microbiota is composed of three groups of bacteria: 1) symbionts that exert health-promoting effects, 2) commensals that exert a neutral effect on the host, and 3) pathobionts that could be pathogenic. The composition of these groups is well balanced in healthy gut microbiota [114]. Dysbiosis refers to a change in the composition of the gut microbiota in comparison to healthy or control microbiome, which is attributed to a reduced number of symbionts and commensals and/or increased number of pathobionts. As mentioned above, several factors alter the gut microbe balance, thus creating dysbiosis. Diseases related to dysbiosis are, for example, autoimmune diseases, metabolic diseases, bacterial infections, and cancers. Growing evidence indicates that several diseases and disorders may alter the composition of gut microbiota [115], for example: esophageal cancer [116], type 2 diabetes [71] , liver and lung diseases [117, 118], obesity [119], psychiatric diseases [120, 121], multiple sclerosis [122], and Alzheimer's disease [121].

\section{Abbreviations}

$A \beta$ - $\beta$-amyloid; AD - Alzheimer's disease; ADHD - Attention-deficit-hyperactive disorder; AIEC Adherent-invasive E. coli; AITDs - Autoimmune thyroid diseases; AMPK - AMP-activated protein kinase; AN - Anorexia nervosa; ASD - Autism spectrum disorders; BD - Bipolar disorder; BE Barrett's esophagus; BPAD - Bipolar affective disorder; CCA - Cholangiocarcinoma; CD - Crohn's disease; CNS - Central nervous system; CRC - Colorectal cancer; EAC - Esophageal adenocarcinoma; EC - Esophageal cancer; GC - Gastric cancer; GD - Graves' disease; GERD - Gastroesophageal reflux 
disease; HCC - Hepatocellular carcinoma; HT - Hashimoto's thyroiditis; IBD - Inflammatory bowel disease; IL - Interleukin; IPMNs - Intraductal papillary mucinous neoplasms; LN - Lupus nephritis; LPL - Lipoprotein lipase; LPS - Lipopolysaccharide; MS - Multiple sclerosis; OSCC - Oral squamous cell carcinoma; PCNs - Pancreatic cystic neoplasms; PD - Parkinson's disease; PDAC - Pancreatic ductal adenocarcinoma; PKC- $\delta$ - Protein kinase- $\delta$; RA - Rheumatoid arthritis; SD -Senile plaques; SIBO - Small intestinal bacterial overgrowth; SLE - Systemic lupus erythematosus; TSHR - Thyroidstimulating hormone receptor; T1DM - Type 1 diabetes mellitus; T2DM - Type 2 diabetes mellitus; UC - Ulcerative colitis

\subsection{Dysbiosis and Autoimmune Diseases}

Intestinal mucosa acts as an important barrier against pathogen invasion. The intestinal wall consists of a layer of mucus, IgA secretory cells, antimicrobial peptides, as well as adhesion and tight junctions. These compounds form an epithelial barrier [123]. However, gut microbiota can change the mucosal immunity by modulating the immune responses and consequently, autoimmunity [124].

Type 1 diabetes mellitus (T1DM) is an autoimmune disease characterized by the destruction of insulin-producing pancreatic $\beta$-cells in the islets of Langerhans. Autoantibodies against glutamic acid decarboxylase (GAD-65), tyrosine phosphatase-2 (IA-2), insulin (IAA), as well as autoreactive CD4+ and CD8+ T cells are present in patients with T1DM. In 1987, Suzuki et al. in their animal study, suggested the effect of gut microbiota on the development of T1DM [125]. This was further confirmed in human and animal studies. It was found in a case-control study in Finland that the gut microbiota of healthy children differed from that of patients with T1DM, and healthy children had more diverse and stable microbiota [126]. In children who developed T1DM, the Firmicutes count reduced, whereas that of Bacteroidetes increased. In another study, children with T1DM had higher counts of Clostridium, Bacteroides, and Veillonella and lower counts of Bifidobacterium and Lactobacillus [127], increased count of Bacteroides ovatus and decreased count of B. fragilis. In children with T1DM, a lower abundance of lactate- and butyrate-producing bacterial species (Bifidobacterium adolescentis and B. pseudocatenulatum) and increased abundance of Bacteroides genus was observed as compared to those in children without T1DM [128]. Similar changes in the gut microbiota were observed in Mexican children [129]. In children with autoantibodies against $\beta$ cells, a higher count of Bacteroidetes phylum and Bacteroides genus and lower count of butyrateproducing bacteria, such as Clostridium clusters IV and XIV a [130] were observed, as reviewed by Bibbo et al. [131].

Mucin plays a critical role in maintaining gut integrity. Its synthesis is induced by lactate- and butyrate-producing bacteria, whereas its synthesis is impaired by non-butyrate-producing, lactate utilizing bacteria. Disturbances in the mucin synthesis and disruption of the intestine barrier may result in $\beta$-cell autoimmunity and T1DM [132]. The activity of gram-positive bacterial species of Lactobacillus (L. acidophilus, L. fermentum, L. gasseri, and L. rhamnosus) can change the expression of genes encoding for junction and adhesion proteins $E$-cadherin and $\beta$-catenin, as well as downregulate the expression of protein kinase $C-\delta$ (PKC- $\delta$ ) [133]. The activation of PKC- $\delta$ can disrupt adherence and increase intestinal permeability [134]. For instance, ultra-structural alterations and increased intestinal permeability were observed in patients with T1DM [135]. Results obtained by Bosi et al. [136] reported that increased gut permeability preceded the clinical onset of T1DM. However, their studies suggest that increased permeability alone could induce T1DM [137]. As 
summarized by Gülden et al. [137], “... gut permeability may be an important player in the development of T1DM, but, yet, the findings in human studies have shown association, but causation will be more difficult to prove" [137]. Increased permeability facilitates the absorption of antigens, resulting in the injury of pancreatic $\beta$-cells [138] and triggering immune responses. Most information on the role of gut microbiota in the development of T1DM has been obtained from animal studies. The components of the bacterial cell wall, for example, LPS, flagellin, and polysaccharide A (PSA), activate the innate immune response [139-141]. They can alter the development and function of lymphocytes. Bacterial components induce the proinflammatory signals, culminating in the production of inflammatory T cells: Th1, Th2, and Th17 that function with IL-1, IL-6, and IL-12 to result in autoimmunity [142]. Both Th1 and Th2 T cells play a significant role in destroying the pancreatic $\beta$-cells. According to Round and Mazmanian [143], Th1 cells may invade pancreatic islets, and Th2 cells may initiate $\beta$-cell destruction. The gut epithelial cells express microbe-associated molecular pattern (MAMP) receptors, principally TLRs. Activation of these receptors induces a proinflammatory response that activates the NF-KB pathway, as well as the production of cytokines, chemokines, and antibacterial products [141]. LPS inhibits IL-1 receptorassociated kinase M (IRAK M), which acts as a modulator of IRAK1, necessary for NF-KB activation. Activation of TLR4 by LPS stimulates the differentiation of effector Th1, Th2, and Th17 cells, development of regulatory T cells (Treg), and production of secretory immunoglobulin A (for details see [141]). Th17 T cells induce pancreatic inflammation. Differentiation of Th17 T cells into Th1 is involved in the development of type 1 diabetes. Therefore, differentiated Th17 phenotypes may inhibit diabetogenic phenotype [144]. Gram-positive bacteria induce Th17 cell differentiation into cells that protect the host from fungal and bacterial infections. This is attributed to the secretion of IL-17, IL-21, and IL-22 by these cells [145, 146]. Furthermore, IL-22 activates epithelial cell tight junction and induces the production of mucin and antimicrobial proteins [114]. However, Th17 cells play a protective role in the development of different diseases, such as type 1 diabetes. Strains of Clostridia (clusters IV, XIVa, and XVIII) and Bacteroidetes enhance the abundance of CD4 ${ }^{+}$Foxp $3^{+}$ regulatory $T$ cells $[31,147]$. These immunosuppressive $T$ cells suppress aggressive immune responses to auto- and bacterial antigens. Moreover, these cells promote epithelial cell repair by activation of NF-KB-dependent signaling pathways [148, 149].

Rheumatoid arthritis (RA) is a chronic autoimmune disorder characterized by inflammation and hyperplasia of synovial joints. It is caused by the production of autoantibody rheumatoid factor and anti-citrullinated protein antibodies [150]. Bacterial DNA and cell wall components have been found in the joints of patients with RA, suggesting that bacteria may play a role in the pathogenesis of RA.

In patients with early RA, decreased abundance of Bifidobacterium, Bacteroides, Group XIV Clostridia, and Lachnospiraceae [151-153] has been observed, whereas levels of $C$. asparagiforme, Gordonibacter pamelaeae, Eggerthella lenta, Bifidobacterium dentium, Ruminococcus lactaris, Lactobacillus salivarius, L. iners, L. ruminis, and Prevotella copri increased in these patients as compared to healthy controls [153-155]. Bifidobacteria, P. copri and B. fragilis, are treated as human commensal bacteria. It was found that $P$. copri induced RA [156], implying that the commensal bacteria may have a pathogenic or protective influence in this disease [130].

A study observed increased microbial diversity and abundance in patients with RA [155]. However, results from other studies report decreased gut microbiota diversity in patients with RA as compared to healthy controls. This correlates with disease duration and levels of serum autoantibodies. In these patients, an expansion of Actinobacteria, which is a rare taxon in the 
human intestine [157], was observed. Based on the obtained results, Chen et al. [158] suggested that three genera Collinsella, Eggerthella, and Faecalibacterium, distinguished patients with RA. Genus Collinsella stimulates the production of proinflammatory cytokine IL-17A by the host and alters gut permeability and disease severity [158]. These results were confirmed in animal studies. In germ-free animal models, RA may be induced by bacteria, such as Lactobacillus species and segmented filamentous bacteria [159]. These bacteria stimulate Th17 and decrease the activity of Treg cells [159]. Lactobacillus spp. bind to the mucosal barrier of the gut and increase in abundance and diversity in patients with RA in the early stage [155]. Based on the obtained results, it is suggested that the bacteria, such as Mycoplasma fermentans [160], E. coli [161], and Proteus mirabilis [162] could be responsible for the initiation of RA. In contrast, other studies suggest that infections of the gastrointestinal tract and urogenital organs are associated with a significantly lower risk of RA [163]. Moreover, patients with RA have decreased gut microbial diversity in comparison with controls [157, 164].

Administration of Lactobacillus casei 01 as a probiotic in patients with RA resulted in significantly low disease activity score [165], decreased serum levels of proinflammatory cytokines, such as TNF- $\alpha$, IL-6, and IL-12, whereas it increased the serum levels of IL-10 as compared to the placebo control patients $[166,167]$.

The human oral cavity consists of over 700 bacterial species [168]. Microbiota of a healthy human contains bacteria, such as Streptococcus, Veillonella, Prevotella, and Haemophilus, whereas gingival plaque samples contain Corynebacterium as the predominant genus [169]. Dysbiosis was detected in the oral cavity in patients with RA. Observed differences in the oral profile between patients with RA and healthy control are probably attributed to the severity of periodontitis but not RA status [170]. Moreover, another study observed differences in the composition of mouth microbes between patients with RA and healthy control [171]. In patients with RA, an abundance of Veillonella and anaerobic bacteria, such as Lactobacillus salivarius and Atopobium spp. were reported. Healthy controls showed an increased number of Haemophilus species, aerobic species, and Porphyromonas gingivalis [172]. Furthermore, an increased level of Cryptobacterium curtum and decreased levels of Neisseria spp. and Rothia aeria were observed [153].

In patients with RA, a higher prevalence of periodontitis (PD), inflammatory damage of the connective tissue and bone of the tooth was observed. This could be attributed to the immune response to microbial plaque [173]. In patients with early RA, there are two characteristic pathogenic bacteria: subgingival Tannerella forsythia and supragingival Streptococcus anginosus [174]. In patients with PD, P. gingivalis, a pathogenic bacterium that belongs to the pathogenic group called "red complex bacteria," was reported. This group also contains Treponema denticola and T. forsythia [175].

Inflammatory bowel disease (IBD) is not a classical autoimmune disorder. It is associated with changes in the composition of the gut microbiota and is characterized by disturbances in the host response against microbiota [176]. IBD includes Crohn's disease (CD) and ulcerative colitis (UC). These diseases are characterized by chronic inflammation of the small bowel and/or colon [177].

There are studies that suggest that autoimmune reactions play a role in the pathogenesis of IBD. Autoimmune reactions are attributed to bacterial-host mimicry [178]. The obtained results revealed that the majority of mimickers are pathogenic bacteria, such as Mycobacteria spp., Campylobacter, and Klebsiella [149]. Patients with IBD often benefit from the treatment with antibiotics, suggesting that bacteria play an important role in the pathogenesis of this disease, and 
IBD is a consequence of an abnormal immune response to bacterial components of the gut microbiota [179]. However, it is still unclear whether the observed alterations in the composition of gut microbiota are the cause or consequence of IBD [178] and requires further investigation [114]. However, in patients with IBD, antibodies directed against a variety of microbial antigens derived from intestinal bacteria have been detected. The detected antibodies were directed against bacteria, such as E. coli, Pseudomonas fluorescens, and yeast (Saccharomyces). This observation may suggest that these microorganisms may induce immune responses [180].

The association of gut microbial dysbiosis with the pathogenesis of IBD is well established. In patients with IBD, the counts of E. coli, bifidobacterial, and Lactobacillus are increased as compared to healthy control subjects [181-183]. In contrast, the levels of Bacteroides and Firmicutes (in particular F. prausnitzii) decreased in these patients [180, 184, 185]. Results obtained from several studies revealed decreased microbial diversity, especially of phyla Firmicutes and Bacteroidetes [180, $184,186]$. The reduction was primarily observed in the number of bacteria, such as $F$. prausnitzii, which, as observed in animal studies, exerts anti-inflammatory functions [185]. In contrast, increased numbers of Proteobacteria and Actinobacteria have been reported in patients with active IBD $[149,180,187]$. In patients with CD, increased numbers of certain strains of $E$. coli, which are associated with intestinal epithelial cells, have been reported [178, 180, 186]. These adherentinvasive $E$. coli (AIEC) adhere to and invade intestinal epithelial cells (IECS), as well as survive and replicate within macrophages $[181,188]$. An increased tendency of genetic defect(s) in the host to kill intracellular bacteria [189] may cause the development and/or progression of chronic intestinal inflammation [180]. According to Png et al. [190], an increased number of Ruminococcus gnavus and $R$. torques, mucolytic bacteria, may facilitate the adhesion and invasion of AIEC. A study revealed an increased abundance of Enterobacteriaceae, Pasteurellaceae, Veillonellaceae, and Fusobacteriaceae in patients with CD, whereas the abundance of Erysipelotrichales, Bacteroidales, and Clostridiales decreased as compared to healthy controls [191].

Reports also suggested that other bacteria may be involved in the pathogenesis of IBD. In 1913, the role of Mycobacterium avium paratuberculosis was shown in the development of CD [177]. However, the role of this bacteria has remained uncertain [192]. Moreover, H. pylori was postulated as a pathogen in the development of IBD [177]. In contrast to these studies, the role of Campylobacter spp. in the etiology of IBD was confirmed in several studies $[193,194]$ The role in the development and/or progression of IBD has also been postulated in several other bacteria, such as Fusobacterium varium [195], Klebsiella pneumoniae [196], Salmonella [197], and Yersinia enterocolitica [198].

Commensal bacteria, such as Bifidobacterium, Bacteroides, Clostridium, and Lactobacillus protect against inflammatory/autoimmune diseases. Therefore, the restoration of the microbial balance in patients with IBD via administration of probiotic VSL\#3, may be a promising therapeutic strategy [199].

Animal studies revealed that microbiota induces inflammation in animal models of colitis due to the activation of T cells involved in innate and adaptive immune mechanisms [200]. A study reported that bacterial species, such as Helicobacter hepaticus, B. fragilis, and B. vulgatus, isolated from the intestine of patients with IBD are capable of inducing intestinal inflammation in rodents [201]. This effect was observed only in immunodeficient mice [201]. Oral administration of $B$. vulgatus, isolated from patients with IBD, induced ulcerative colitis in guinea pigs, whereas those isolated from healthy subjects did not [202]. The proliferation of T cells requires the presence of the microbiota. Bacteria 
can induce the production of IL- 6 by dendritic cells, a step required to reach the threshold number of interferon- $\gamma$ - and IL-17-producing microbiota-specific T cells. These processes induce ulcerative colitis [200].

Polymorphisms in genes, such as NOD2, ATG16L1, and IRGM, which are involved in the killing of bacteria, have been identified in patients with $C D$. Therefore, researchers suggest that inappropriate innate immune responses to intestinal microbiota may promote chronic gut inflammation in individuals who are genetically susceptible [180, 181, 189, 203].

Systemic lupus erythematosus (SLE) is a complex heterogenous autoimmune disorder, which involves a variety of organs and tissues. Unfortunately, the relationship between SLE and gut microbiota is still not well characterized. These observations have been limited to the gut [173].

A study reported an increase in the relative abundance of Bacteroidetes and an over two-fold reduction in the Firmicutes/Bacteroidetes ratio in patients with SLE, as compared with healthy controls, whereas no significant difference in the bacterial diversity was observed [204]. In contrast, Luo et al. [205] reported that the ratio Firmicutes/Bacteroidetes was not significantly lower in patients with SLE in comparison to healthy controls. Moreover, researchers observed differences in the composition of several bacterial species within the genera Odoribacter and Blautia, as well as in the family Rikenellaceae between patients with SLE and healthy subjects. In patients with SLE, the bacterial diversity was lower, whereas the levels of gram-negative bacteria increased [205]. Dysbiosis in the gut microbiota in patients with SLE was also described by Rojo et al. [206]. In patients with SLE, circulating levels of interferon- $\gamma$ correlated negatively with the abundance of Bacteroidetes, and positively correlated with the abundance of Firmicutes and the Firmicutes/Bacteroidetes ratio. These correlations were not observed in healthy controls [173]. In patients with SLE, significant negative correlations were observed between the phylum Synergistetes and the titer of anti-dsDNA antibodies and serum level of IL-6, whereas a positive correlation was observed between these bacteria and total IgM and anti-phosphorylcholine IgM [207]. A lower count of bacteria belonging to the family Lactobacillaceae, exhibiting antiinflammatory functions by inducing Treg cells, was reported in the gut microbiota of patients with SLE [208].

Approximately $60 \%$ of patients with SLE suffer from lupus nephritis (LN) complications [209]. A study using animal models of LN reported a lower Firmicutes/Bacteroidetes ratio. Moreover, the study also suggested the role of Lactobacillus in the pathogenesis of $\operatorname{LN}[209,210]$.

Changes in the composition of gut microbiota due to SLE were confirmed using animal studies [164]. The relative abundance of Lactobacilli, Lachnospiraceae, and Clostridiaceae depends on the progression of the disease in the gut of female lupus-prone mice [211]. Administration of Lactobacillus reuteri in two mouse models of SLE prevented the development of SLE, increased the survival and levels of peripheral Treg cells [130]. In these mouse models, the levels of Treg cells were impaired [212]. These results suggested that the administration of lactobacilli changed the composition of gut microbiota, and therefore protected against the development of SLE by stimulating Treg cells. However, contradictory results on the effect of the composition of gut microbiota on the progression of SLE also exist. For example, the presence of segmented filamentous bacteria in the intestine of a mouse model of SLE (lupus-prone SNF1) was shown in a study to be related to the development of lupus in these mice [213], whereas another study reported no relation of SFB with the outcome of the disease [214]. Therefore, this requires further investigation [130]. 
Autoimmune thyroid disease. There are several autoimmune thyroid diseases (AITDs), the most prevalent of which is Hashimoto's thyroiditis (HT) and Graves' disease (GD). Other autoimmune thyroid diseases include painless thyroiditis (PT), postpartum thyroiditis (PPT), and subacute thyroiditis (SAT) [215]. AITD is frequently associated with other autoimmune diseases, such as Sjögren's syndrome, SLE, and RA [215].

Hashimoto's thyroiditis is also known as autoimmune or chronic lymphocytic thyroiditis [216]. Its pathology involves the interaction of a predisposing genotype with endogenous and environmental factors. Several studies have indicated that dysbiosis of the gut microbiota may be involved in HT pathogenesis. In 1988, animal studies revealed that the transfer of gut microbiota received from conventional rats to specific pathogen-free rats caused increased susceptibility to experimental autoimmune thyroiditis [217]. This observation suggested the possible role of the microbiota in influencing the susceptibility to autoimmune thyroiditis. Certain components of Bifidobacterium and Lactobacillus share amino acid sequences with human thyroid peroxidase and thyroglobulin and selectively bind to human autoantibodies [218]. In contrast, animal studies have revealed that the administration of probiotic Lactobacillus rhamnosus HNOO1 and Bifidobacterium lactis HN019 did not induce experimental autoimmune thyroiditis in these mice [219]. Moreover, it is postulated that HT could occur due to immune activation by LPS derived from the cell wall of gram-negative bacteria [220].

The composition of microbiota in fecal samples of patients with HT and healthy controls revealed similar levels of bacterial richness and diversity in both HT patients and healthy subjects. Increased levels of the following genera: Blautia, Roseburia, Ruminococcus torques group, Romboutsia, Dorea, Fusicatenibacter, and Eubacterium halli group were found in patients with $\mathrm{HT}$, whereas the levels of genera Faecalibacterium, Bacteroides, Prevotella_9, and Lachnoclostridium decreased as compared to healthy controls. These differences in the composition of gut microbiota strongly correlated with clinical parameters. Therefore, according to the authors' suggestion, the composition of gut microbiota could be used for disease diagnosis [221]. The role of dysbiosis of gut microbiota in the pathogenesis of HT was confirmed by another study that revealed a similar diversification of gut microbiota in patients with $\mathrm{HT}$ in comparison to healthy controls. Moreover, researchers have reported an abundance of Prevotella_9 and Dialister, an increased number of the genera of the diseased group, Escherichia-Shigella and Parasutterella [222].

Other changes in the composition of gut microbiota between hypothyroid patients and healthy subjects include the following: an association between bacterial overgrowth in the small intestine and hyperthyroidism was reported, and authors postulate that changes in the neuromuscular function of these patients could be attributed to bacterial excess [223]. The abundance of Bifidobacterium, Lactobacillus, Clostridium, and Enterococcus is higher in patients with HT as compared to healthy controls. For example, Clostridium butyricum $[224,225]$ and Lactobacillus are considered probiotics. For more details on the role of probiotics in HT, please refer to these studies $[226,227]$. It should be noted that the gut microbiota also plays a beneficial role in thyroid hormone metabolism [228].

Graves' disease (GD) is an autoimmune disease. Like other autoimmune diseases, its pathogenesis is attributed to interpreting self-antigens as foreign or harmful antigens [229]. The immune system in patients with GD cannot differentiate between foreign antigens and the thyroid tissue. Therefore, the thyroid gland is destroyed by body's immune response, which recognizes the 
thyroid-stimulating hormone receptor (TSHR) as an autoantigen, thus producing autoantibodies against TSHR [230].

It is suggested that GD could be due to gut microbial dysbiosis [229]. Researchers have reported lesser diversification of the gut microbiota in patients with GD in comparison to healthy subjects. In patients with GD, a significantly higher abundance of Prevotellaceae and Pasteurellaceae, and significantly lower abundance of Enterobacteriaceae, Veillonellaceae, and Rickenellaceae were observed as compared to healthy controls. At the genus level, in the diseased group, a significantly higher abundance of Prevotella_9 and Haemophilus, and significantly lower abundance of Alistipes were present in comparison to control subjects [229]. Moreover, antibodies against Yersinia enterocolitica and Helicobacter pylori were detected; however, these responses were not observed in all patients with GD [216]. Unfortunately, there exists a lack of investigations on the role of microbial dysbiosis in other autoimmune thyroid diseases.

\subsection{Dysbiosis and other Inflammatory and Autoimmune Diseases}

Inflammatory diseases include allergic disorders. The interaction between the gut microbiota and these diseases is poorly characterized.

It is reported that the gut microbiota is altered in infants with food allergies and eczema, in comparison to healthy subjects. Lower levels of lactobacilli and bifidobacteria were found in children with allergies; however, levels of coliforms and S. aureus were higher, as compared to in children without allergies [231]. The decreased levels of bifidobacteria and Enterococcus and increased levels of Clostridium were observed in infants with atopic eczema [232, 233]. An important and interesting observation is the colonization of the gut by lactobacilli at one week of life, which decreases the risk of allergy for five years of age [234]. Controversial results were obtained from studies on infants with asthma. For instance, one study reported high levels of Clostridium species as protective [235]. Another study reported that the colonization of the gut by C. difficile in 1-month-old infants could cause asthma at 6 to 7 years of age [236].

In addition to the above-mentioned autoimmune diseases, the dysbiosis of gut microbiota may result in several other autoimmune diseases. An association was found between gut microbiota dysbiosis and development and/or progression of diseases such as: ankylosing spondylitis, psoriatic arthritis, Sjögren's syndrome, irritable bowel syndrome, psoriasis, vasculitides, and atopic dermatitis. In contrast, it is not completely understood if these organisms play a specific role in the development and progression of mentioned diseases. There are limited reports on the association of these diseases and dysbiosis, and obtained results are sometimes controversial, as well as the interactions of gut microbiota and the above-mentioned inflammatory and autoimmune diseases are poorly characterized. Moreover, an important question is: Is dysbiosis a cause or effect of a disease?

\subsection{Dysbiosis and Metabolic Disorders}

Dysbiosis of human microbiota may also cause metabolic disorders such as obesity and type 2 diabetes mellitus.

Obesity has become a pandemic-like situation and is associated with several metabolic changes. Several accumulating data suggest an association between gut microbiota and the development of 
adiposity. Therefore, dysbiosis of gut microbiota is treated as a risk factor in the obesity epidemic [237].

The first report of changes in the gut microbiota was described between obese and lean mice with a mutation in the leptin gene $(o b / o b)$ [52]. Researchers observed a significant reduction in Bacteroidetes and an increased number of Firmicutes in obese (ob/ob) mice as compared to lean mice. Other results revealed that the most common class among Firmicutes phyla was Mollicuses in obese mice [238]. Similar observations were performed in humans. In obese subjects, the number of Firmicutes increased, whereas the proportional increase in the number of Bacteroidetes reduced the weight [239]. The increase in the number of Firmicutes by $20 \%$ and decrease by $20 \%$ in the number Bacteroidetes suggested that the amount of energy obtained from the food increased by $150 \mathrm{kcal}$ [240]. Species belonging to both Firmicutes (S. aureus) and Bacteroidetes (Bacteroides/Prevotella) were observed in overweight women [241]. Moreover, increased levels of S. aureus were observed in overweight children [242], and significantly higher levels of $F$. prausnitzii (phylum Firmicutes) were detected in obese Indian children in comparison with non-obese children [243]. The number of Bacteroides decreased in overweight pregnant women, whereas the number of Staphylococcus, Enterobacteriaceae, and E. coli increased in comparison to pregnant women with normal weight [244]. Increased proportions of the Bacteroides-Prevotella [245] was reported in obese adolescents after weight loss. In contrast, a study reported a lack of associations between body weight and the ratio Bacteroidetes: Firmicutes in the human intestine [246]. Similarly, another study revealed a higher proportion of Bacteroidetes in overweight and obese subjects [247]. These differences could be attributed to the different methods used in the studies [248]. Moreover, it also remains unclear whether changes in the composition of human gut microbiota in obese subjects are a result or cause of obesity [84]. A decreased number of Firmicutes and/or increased proportions of Bacteroidetes was observed in obese patients with type 2 diabetes following Roux-en-Y gastric bypass [249].

The association between obesity and the composition of intestinal microbiota was confirmed in several other animal studies. For example, it was observed in these studies that normalization of proportion of $A$. muciniphila caused an improvement in several metabolic disorders such as fat mass gain, metabolic endotoxemia, and insulin resistance [250, 251].

Lipopolysaccharide (LPS), a compound present in gram-negative bacterial cell walls, plays an important role in obesity. Increased plasma LPS levels, defined as metabolic endotoxemia, triggers chronic inflammation associated with obesity [103]. LPS binds to TLR4 and initiates inflammatory events such as secretion of proinflammatory cytokines (IL-6 and TNF- $\alpha$ ). Based on the results obtained from animal studies, the development of obesity could be attributed to suppression of fasting-induced adipose factor (FIAF), also known as angiopoietin-like protein 4 (Angptl4), by gut microbiota. FIAF is an inhibitor of lipoprotein lipase (LPL). LPL hydrolyzes triglycerides and stimulates their storage in the adipocytes. Suppression of FIAF increases the activity of LPL and lipid storage, thus stimulating the deposition of triglycerides in fat cells [252,253]. Moreover, it is suggested that gut microbiota enhances gut permeability, resulting in increased LPS plasma levels and the appearance of hyperphagia and obesity [103]. Downregulation of T1DM (AMPK) expression by intestinal microbiota inhibits the oxidation of fatty acids, resulting in obesity [253]. Furthermore, the endocannabinoid system has been reported to regulate the gut barrier function during obesity [109]. 
Animal studies have revealed that intake of prebiotics alters the composition of 102 different taxa in obese mice, a model of type 2 diabetes. Administered therapy resulted in lower accumulation of fat mass, increased muscle mass, and improved metabolism of glucose and lipids [251]. Reduced gut permeability, metabolic endotoxemia, and whole-body inflammation were observed in these mice. Prebiotics stimulate the synthesis and secretion of gut peptides, such as GLP-1 and GLP-2, in the proximal colon, and increase the number of enteroendocrine cells ( $L$ cells) that produce these gut peptides [251]. Therefore, the authors conclude that targeting endocrine function may be a therapeutic strategy against metabolic inflammation associated with obesity and type 2 diabetes.

In humans, consumption of prebiotics alters the composition of gut microbiota, increases plasma levels of GLP-1 [86, 254, 255], lowers postprandial glycemia [86], increases satiety, decreases hunger and energy intake, and reduces visceral fat mass [86, 254, 255].

Type 2 diabetes mellitus (T2DM) is a prevalent metabolic disease worldwide, and obesity is associated with its development. As described above, dysbiosis in the gut microbiota influences the development of obesity, and therefore, it is not surprising that the composition of the gut microbiota might also influence T2DM.

Specific changes in the gut microbiota are observed in patients with T2DM. For example, the levels of Bacteroides and Prevotella are increased, whereas levels of those belonging to the phylum Firmicutes and class Clostridia decreased proportionally. A decrease in anti-inflammatory bacteria, such as bifidobacterial, was observed in another study $[39,40]$. Moreover, specific changes in the composition of the gut microbiota in each progressive stage, leading to the development of diabetes, was observed [256]. The relative abundance of $A$. muciniphila and F. prausnitzii, a butyrateproducing bacteria, and Verrucomicrobia decreased with the decrease in glucose tolerance, with increased levels of Betaproteobacteria. Chinese diabetic patients exhibited only a moderate degree of intestinal dysbiosis. The levels of butyrate-producing bacteria, such as Roseburia intestinalis and F. prausnitzii, decreased, whereas those of several opportunistic pathogens, such as Bacteroides caccae, Clostridium hathewayi, C. ramosum, C. symbiosum, E. lenta, and E. coli - increased, as compared with the healthy controls. In patients with T2DM, sulfate-reducing species Desulfovibrio and the mucin-degrading A. muciniphila were frequently detected [256], whereas in the healthy subjects, butyrate-producing bacteria, such as $F$. prausnitzii, $R$. intestinalis, and others, were enriched. A study from Europe revealed that in postmenopausal women with T2DM, the abundance of Lactobacillus gasseri, Streptococcus mutans, and Clostridium clostridioforme increased. In contrast, decreased levels of at least five other Clostridium species, R. intestinalis, and F. prausnitzii were observed in these women as compared with healthy controls [257]. Furthermore, changes in the composition of human gut microbiota in patients with T2DM were described by other study groups $[258,259]$. Several results obtained in human clinical studies were confirmed in animal studies [260].

Several mechanisms have been proposed to explain the crosslink between gut microbiota and T2DM. One of these suggested mechanisms is metabolic endotoxemia due to LPS, which could be involved in chronic inflammation observed in patients with T2DM. Animal studies have revealed that changes in the gut microbiota, due to HFD, increased the LPS levels and changed the grade of adipose tissue inflammation [77, 261]. The role of LPS in the development of T2DM was confirmed in animal studies. Injection of LPS to mice with the genetic absence of CD14/TLR4 receptor did not result in the development of metabolic disturbances and T2DM [77]. 
Excessive intake of fructose is another dietary pattern involved in metabolic disorders and endotoxemia. A high-fructose diet fed to mice led to a 27-fold increase in portal endotoxin levels, resulting in a significant increase in plasma inflammatory cytokines and insulin resistance, as compared with water control [262].

Animal studies revealed that the administration of prebiotics that increased the numbers of Bifidobacterium, modulated inflammation in obese mice by increasing the intestinal secretion of GLP-1 and PYY. These molecules decreased insulin resistance and increased the functionality of $\beta$ cells [95]. Modulation of the gut microbiota due to prebiotics increased the production of GLP-2 in the colon, increasing the expression of zonula occludens-1. It improved the mucosal barrier function by reducing the intestinal permeability and leading to decreased levels of plasma LPS [263]. In patients with type 2 diabetes, decreased number of butyrate-producing bacteria were observed [256]. The elevated levels of butyrate-producing bacteria exert a protective role against functional dysbiosis because butyrate is the preferred source of energy, and repair and maintain cell health in the human digestive system.

\subsection{Dysbiosis and Psychiatric Disorders}

Autism spectrum disorders (ASD) are a heterogeneous group of neurodevelopmental disorders. These are neuropsychological disorders in which the gut microbiota plays a key role. There has been growing evidence of microbial dysbiosis in ASD [264-266]; however, the possible mechanisms of this link remain unknown [267].

The association of ASD with gut microbiota began with a study on Clostridium, and the hypothesis that it plays a role in the etiology of ASD[268]. Clostridium is a common bacterium in the gastrointestinal tract, with significantly higher levels in patients with ASD [269]. Clostridium tetan produces the tetanus neurotoxin (TeNT), which may be transported by the vagus nerve. TeNT inhibits the release of neurotransmitters by proteolytic cleavage of synaptobrevin, resulting in behavioral disruptions observed in ASD [268]. The post-mortem analysis of the cerebellum of patients with ASD revealed a decreased number of Purkinje cells [268], producers of GABA, that are vulnerable to the TeNT[268]. Moreover, Clostridium releases a metabolic product, 3-(3hydroxyphenyl)-3-hydroxypropionic acid (HPHPA), which is specific to this genus and responsible for emptying and depleting catecholamines in the brain, thus inducing autism symptoms [270]. In fecal samples of children with ASD, a high abundance of Clostridium clusters I and XI, which contain C. perfringens and $C$. difficile, both species are known to produce the toxin, were found with reduced levels of the cluster XIVab. The cluster XIVab contains beneficial bacteria [271, 272]. Clostridia are propionate producers, and animal studies revealed that propionic acid impairs social behavior in rats [273].

Investigations of Indian children with ASD showed a higher relative abundance of families Lactobacillaceae, Bifidobacteriaceae, and Veillonellaceae. The family Prevotellaceae is predominantly present in healthy children. The obtained results in these studies showed that the prevalence of beneficial bacteria in Indian children with ASD had considerably lower levels of Bifidobacterium, slightly lower levels of Enterococcus, and significantly higher levels of Lactobacillus [274], as compared with healthy controls. In fecal samples of children with ASD, compared with healthy children, the gut microbiota composition was less diverse, with reduced levels of Prevotella, Coprococcus, and Veillonellaceae - bacteria responsible for carbohydrate ingestion and 
fermentation. At the phylum level, a higher abundance of Firmicutes and a lower abundance of Bacteroidetes, Actinobacteria, and Proteobacteria were present [271]. However, in another study, lower levels of Firmicutes in fecal samples of children with ASD were observed [275]. In these children, an increased ratio of Firmicutes: Bacteroidetes was found, with higher levels of Lactobacillus and Desulfovibrio species $[276,277]$. The abundance of Desulfovibrio positively related to the severity of ASD [277]. In fecal samples of patients with ASD, increased levels of Sutterella and Ruminococcus torques, bacteria that are associated with the mucosa [278], were observed and those belonging to genera Lactobacillus, Clostridium, Desulfovibrio, Caloramater, Alistipes, Sarcina, A. muciniphila, Anaerofilum, Barnesiella intestinihominis, Dorea, F. prausnitzii, Parasutterella excrementihominis, Prevotella copri, P. oris, Turicibacter sanguinis, as well as families of Sutterellaceae and Enterobacteriaceae [275, 279-281]. In contrast, in autistic children, in comparison with healthy controls, decreased levels of Eubacterium siraeum, E. coli, Collinsella aerofaciens, Enterococcus, Lactobacillus, Lactococcus, Staphylococcus, Bifidobacterium, Blautia, and Dialister were observed [280, 281].

Samples obtained from the intestinal biopsies of the cecum and terminal ileum showed increased levels of Sutterella spp., as compared with healthy subjects. Higher levels of these bacteria were detected in patients with ASD [282]. An analysis of the duodenal biopsies revealed that levels of genera Burkholderia, Actinomyces, Oscillospira, Peptostreptococcus, and Ralstonia were elevated in patients with ASD, whereas the levels of those belonging to genera Neisseria, Bacteroides, Devosia, Prevotella, Streptococcus, and E. coli decreased in these patients in comparison with healthy subjects [283]. For more details on the association of gut microbiota dysbiosis and ASD, refer to cited studies [265, 266].

Dysbiosis in ASD also involves yeasts; however, their role in ASD has not been clearly elucidated $[284,285]$. Animal studies have revealed that fungal infection causes Th-17 cells to respond and produce cytokine IL-17, which is implicated in the etiology of ASD [286].

Administration of probiotics and fecal microbiota transplants are suggested as potential therapeutics for ASD [287, 288].

Attention-deficit hyperactive disorder (ADHD) is a common, early-onset neurodevelopmental disorder. The etiology and pathophysiological mechanisms of ADHD have remained unclear [289]. However, diet, such as the "Western" diet, has been suggested as a potential predisposing factor for ADHD [290, 291]. Moreover, a change in the diet has been reported to exert positive outcomes on symptoms of ADHD [292].

The composition of gut microbiota in adolescent and adult patients with ADHD differs in comparison with healthy controls [293]. Increased levels of Actinobacteria and reduced levels of Firmicutes have been observed. Researchers attribute these differences to differential regulation and/or synthesis of dopamine precursors. Furthermore, these changes were found to be associated with decreased reward anticipation [293]. Dopamine is a catecholamine that plays a role both as a hormone and a neurotransmitter. It is suggested that a link exists between ADHD and alterations in dopamine metabolism [294]. Bifidobacterium, belonging to the phylum Actinobacteria, may influence the levels of dopamine in the body. This bacterium contains enzyme cyclohexadienyl dehydratase (CDT) [293], which is important for the synthesis of phenylalanine, a precursor of tyrosine, which is metabolized into dopamine and finally to noradrenaline. Increased levels of Bifidobacterium increases the levels of CDT. The authors conclude a negative correlation between the abundance of CDT and reward anticipation, a key symptom in ADHD. Researchers have 
suggested high levels of phenylalanine could be a risk factor for abnormal dopamine signaling, causing reduced reward response [293]. It was found that patients with ADHD had significantly lower levels of Dialister spp., in comparison with healthy controls [295]. Interleukin-6, a proinflammatory cytokine, is inversely associated with Dialister spp., and this bacterium positively correlates with an altered temperament and impulsiveness in toddlers, common symptoms of ADHD [296]. In patients with ADHD, increased levels of proinflammatory cytokines have been observed [297]. Several other differences in the composition of gut microbiota between patients with ADHD and healthy subjects were described. For example, obtained results revealed decreased levels of Bacteroides coprocola in patients with ADHD, whereas the relative abundance of $B$. uniformis, B. ovatus, and Sutterella stercoricanis was higher in these patients in comparison with healthy subjects. Moreover, these bacteria positively correlated with ADHD symptoms. In patients with ADHD, the levels of Fusobacterium and Alistipes were higher, whereas a higher abundance of Lactobacillus, Prevotella, and Escherichia-Shigella was detected in healthy controls [290]. In patients with ADHD, $\alpha$-diversity was significantly lower in comparison with healthy subjects, which could be attributed to bacteria of the family Prevotellaceae and Neisseriaceae [298], whereas $\beta$-diversity was similar in both patients with ADHD and healthy subjects [290].

S. stercoricanis in both patients with ADHD and healthy subjects is significantly associated with the intake of dairy, nuts/seed, legumes, ferritin, and magnesium, whereas the presence of Bacteroides uniformis correlates with the intake of fats and carbohydrates. This observation suggests that the composition of gut microbiota is linked to dietary patterns and susceptibility to ADHD [290]. Moreover, it is suggested that Bifidobacterium, belonging to the phylum Actinobacter, as well as four species Bacteroides uniformis, B. ovatus, B. coprocola, and S. stercoricanis, may serve as potential microbiota markers for $\operatorname{ADHD}[289,290]$. Administration of Lactobacillus rhamnosus as probiotic during the perinatal period showed that probiotic-treated children were less likely to be diagnosed with ADHD [299].

Schizophrenia is a mental disorder in which patients present with delusions, hallucinations, disorganized thoughts and speech, abnormal motor behavior, and negative symptoms. An association of schizophrenia with gastrointestinal comorbidities, such as IBD, IBS, and diseases that are associated with dysbiosis of gut microbiota has been reported. Therefore, it is suggested that the gut microbiota may be involved in the pathogenesis of schizophrenia. Moreover, the involvement of the gut microbiota is suggested based on other observations, such as increased intestinal permeability in patients with schizophrenia [300] and chronic inflammation associated with its progression [301]. Unfortunately, the obtained results are different and controversial. For example, one study revealed a higher abundance of Proteobacteria in patients with schizophrenia in comparison with healthy controls, accounting for predominantly increased levels of Succinivibrio. Based on the obtained results, researchers suggest that Gammaproteobacteria may serve as a biomarker of schizophrenia [302]. Other results showed decreased levels of Proteobacteria in these patients [303] or no major differences at the phylum level [304].

Results from a study suggest that infection by $T$. gondii acts a risk factor for early-onset schizophrenia [305]. Schizophrenia is also associated with bacteriophages, which change the metabolism of bacteria and the composition of the microbial community. For example, an increased load of the Lactobacillus bacteriophage in the oropharyngeal lumen of patients with schizophrenia was reported [306]. 
Probiotics have been proposed as a potential adjunctive treatment for schizophrenia. Unfortunately, only one published study and authors of this study found no effect of probiotic treatment (L. rhamnosus GG and Bifidobacterium animalis subsp. lactis) on the symptoms of schizophrenia [307].

Depression is a mental disorder in which patients present low mood, low self-esteem, and loss of interest in normally enjoyable activities. It is a major form of mood disorder. It was found that the Mediterranean diet enriched with $15 \mathrm{~g}$ of walnuts, $7.5 \mathrm{~g}$ of hazelnuts, and $7.5 \mathrm{~g}$ of almonds per day decreased the risk of depression by $41 \%$ in patients with T2DM, as compared with healthy controls [308].

A study showed strong evidence that gut microbiota plays a role in depression [309]. The association between gut microbiota and depression has been revealed using studies in animal models. In humans, only a few correlations have been demonstrated.

The composition of gut microbiota of patients with depression differs in comparison with that in healthy subjects [310]. However, some findings are contradictory [311]. It is not known whether an altered composition of gut microbiota is a cause or an effect of depression. In fecal samples of patients with depression, increased bacterial $\alpha$-diversity was reported in comparison with healthy controls. The relative abundance of Bacteroidetes, Proteobacteria, and Actinobacteria increased in depressed patients, whereas the level of Firmicutes decreased [310]. The reduced relative abundance of Faecalibacterium [310, 312] was found to have a negative correlation between the severity of depression and the prevalence of Faecalibacterium [310]. In another study, the levels of Bifidobacterium and Lactobacillus were found to be decreased in patients with depression as compared with those in healthy controls [313]. In another study, an elevated abundance of Bacteroidales and lower abundance of Lachnospiraceae family and genera Alistipes and Oscillibacter were found in patients with depression in comparison with healthy controls. Based on the obtained results, the authors conclude that the order Bacteroidales is associated with depression [314].

Animal studies confirmed the association between dysbiosis of gut microbiota and depression $[312,315]$.

The first probiotic treatment for depression was started 1910 [316]. In 2016, the intake of probiotics was observed to result in significant improvement in depressive symptoms [317]. A positive effect on the healthy population and depressed patients was observed only on the participants under 60 years, with no effects of probiotics on people aged above 65 years [318]. A variety of probiotics have been investigated in animal models of neurological disorders [319]. As an antidepressant treatment, no beneficial effect has been found in studied prebiotics [320]. Moreover, a fecal microbiota transplant was suggested in the case of depression; however, it lacked information.

Bipolar disorder (BD), also known as bipolar affective disorder (BPAD), is a psychiatric disorder associated with dysbiosis of gut microbiota. Several studies have been conducted on the composition of the microbiome in patients with $\mathrm{BD}$. In patients with $\mathrm{BD}$, significantly lower levels of Faecalibacterium were detected in comparison with healthy controls [321, 322]. Atypical antipsychotic intake changes the composition of gut microbiota in comparison with non-treated patients. Significantly reduced species diversity was found in treated women as compared with nontreated women [323]. BD is also associated with Flavonifractor, a bacterial genus that may induce oxidative stress and inflammation [324]. This result may suggest an abnormal inflammatory response in bipolar disorder. 
Clinical trials demonstrate a beneficial effect of adjunctive probiotics containing different strains of Lactobacillus or Bifidobacterium in patients with bipolar disorder [325, 326].

Anxiety disorder is a mental disorder in which the main clinical manifestation is anxiety [327]. A small investigation suggested an association of dysbiosis with anxiety [328]; however, a direct link between anxiety and the gut microbiota has not yet been identified. Based on animal studies, researchers suggest that the observed symptoms are due to increased gut permeability [329]. In one study, lack of significant association between anxiety and microbial composition in healthy females was observed [330], whereas reduced microbial richness and diversity in drug-naïve and medicated patients with anxiety disorders, as well as decreased levels of SCFA-producing bacteria, such as Faecalibacterium, Eubacterium rectale, and Sutterella were found in another study [331].

Small cross-sectional studies report the potential of treatment using probiotics for anxiety disorders. Moreover, animal studies have confirmed that Bifidobacterium and Lactobacillus may have beneficial effects on neurological disorders, such as anxiety [319].

Anorexia nervosa (AN) is one of the most common chronic illnesses in female adolescents. Medication is very limited, and psychotherapeutic interventions are only moderately effective. Moreover, not many studies have investigated the association of the gut microbiota with AN during the acute and recovered states. Therefore, our knowledge of the association of the gut microbiota and AN is scarce. In one study, from a single AN sample, 11 new, previously unknown bacterial species were identified [332]. In another study of patients with AN, a reduced number of total bacteria and obligate anaerobes as compared with healthy individuals was detected [333]. An analysis of the fecal samples of females, mostly adult patients with AN, revealed significantly reduced $\alpha$-diversity in these patients [334]. Moreover, increased levels of mucins degraders (e.g., Verrucomicrobia and bifidobacteria) and decreased numbers of butyrate producers (e.g., Roseburia spp.) were observed. E. coli may play a crucial role in the pathogenesis of AN. This bacterium plays a role in the bulimia nervosa and binge-eating disorder, secreting a protein called ClpB, which mimics $\alpha$-melanocyte-stimulating hormone. ClpB is a bacterial heat-shock protein, an antigenmimetic of the anorexigenic peptide $\alpha-\mathrm{MSH}[335]$.

\subsubsection{Gut Microbiota and Neurodegenerative Disorders}

Alzheimer's disease $(A D)$, also known as senile dementia or cognitive disorder, is a neurodegenerative disease of the central nervous system (CNS) in the elderly; it is the most common form of dementia. $A D$ occurs due to the accumulation of extracellular proteinaceous misfolded $\beta$-amyloid $(A \beta)$ fibrils, senile plaques (SPs), and intracellular neurofibrillary tangles [336].

Studies report that gut dysbiosis might be associated with the onset of $\operatorname{AD}[336,337]$. Moreover, this suggestion was confirmed based on the results obtained using the $A \beta$ precursor protein (APP) transgenic mouse model. Authors have found that dysbiosis in the gut microbiota may contribute to amyloid deposition [336].

Glutamate and N-methyl-D-aspartate (NMDA) glutamate receptors play an important role in the physiological functions of the human brain, such as learning and memory [338, 339]. Post-mortem investigations of the brains of patients with $A D$ showed an elevated level of neurotoxic $\beta$-N-methylamino-L-alanine (BMAA) amino acid, which is influenced by NMDA. It is released by cyanobacteria in the human intestine. Moreover, this neurotoxin may be involved in inflammatory neurodegeneration observed in patients with $\operatorname{AD}[338,340]$. Chronic exposure to BMAA triggers the 
formation of intracellular neurofibrillary tangles and $A \beta$ deposits in the brain, increasing the risk of AD [341]. Furthermore, cyanobacteria secrete other neurotoxins, such as saxitoxin and anatoxin- $\alpha$, which may be involved in neurological diseases. Therefore, researchers suggest that the risk of $A D$ may depend on the number of cyanobacteria in the human intestine [342, 343]. Lactobacillus brevis and Bifidobacterium dentium synthesize $\gamma$-aminobutyric acid (GABA), the major inhibitory neurotransmitter in the human CNS. The concentration of GABA in the CNS depends on its concentration in the gastrointestinal tract. Decreased levels of GABA in the CNS are attributed to decreased concentration of GABA in the intestine, as an effect of decreased levels of $L$. brevis and $B$. dentium in the intestine. The dysfunction in CNS due to GABA perturbations could be linked to the development of $A D$. A post-mortem study of patients with $A D$ revealed reduced levels of $G A B A$ in the frontal, temporal, and parietal cortex as compared with patients without AD [344].

Increased risk of AD development could be linked to the infection by Chlamydia pneumoniae. Post-mortem investigations of the brain of patients with $A D$ revealed the presence of these bacteria in the astrocytes, microglia, and neurons. These were detected in senile plaques and intracellular neurofibrillary tangles in infected cells [345]. In another post-mortem investigation of brain samples of patients with $A D$, researchers observed significantly higher levels of Actinobacteria, primarily those of Propionibacteriaceae family, in comparison with control samples. The authors concluded that the migration of bacteria into the CNS could cause neurological diseases or facilitate the pathogenesis of these diseases [346]. Reduced microbial diversity in patients with AD in comparison with healthy subjects was observed. The levels of Firmicutes and Bifidobacterium decreased, whereas those of Bacteroidetes increased [347]. Moreover, an association between infection by $H$. pylori and $A D$ was described. These bacteria release the proinflammatory cytokines and induce oxidative stress, which may stimulate the development of AD [348]. Development AD may be also be stimulated by other bacteria, such as E. coli, Salmonella enterica, S. typhimurium, Bacillus subtilis, Mycobacterium tuberculosis, and $S$. aureus, which produce functional extracellular amyloid fibers $[340,349]$. Results obtained in an animal study using the $A \beta$ precursor protein transgenic mouse model showed decreased levels of Firmicutes, Verrucomicrobia, Proteobacteria, and Actinobacteria, whereas the levels of Bacteroidetes increased [336].

An association between the infection by $T$. gondii, a protozoan intracellular parasite, and the development of $A D$ has been suggested. T. gondii stimulates chronic inflammation in the brain and CNS. In patients with AD, the levels of serum anti-T. gondii antibodies are elevated [350].

Animal studies implicate that the modulation of gut microbiota by probiotics may protect against the progression of $A D$. The administration of probiotic formulation SLAB51, containing Streptococcus thermophilus, Bifidobacterium longum, B. breve, B. infantis, Lactobacillus acidophilus, L. plantarum, L. paracasei, L. delbrueckii subsp. Bulgaricus, and L. brevis in a mice model of AD showed partial restoration of the two impaired neuronal proteolytic pathways (the ubiquitin proteasome system and autophagy). Therefore, the authors suggested that the modulation of gut microbiota may slow down the progression of $A D$ [351].

The administration of prebiotics has been reported to decrease the risk of development and progression of $A D$. Obtained results revealed that people aged 65 to 79 years, who daily drank 3 to 5 cups of coffee at midlife, showed $65 \%$ decreased risk of AD in comparison with people who did not drink or drink less than 2 cups per day [352]. Polyphenols, which contain coffee, reduce oxidative stress. Moreover, coffee influences the composition of gut microbiota. It reduces the ratio of 
Firmicutes to Bacteroidetes, causing reduced inflammation [353]. Further, reports suggest other therapies for the prevention and treatment of AD and other disorders of CNS [354, 355].

Parkinson's disease (PD) is a CNS disease associated with the dysbiosis of gut microbiota. PD is a multicentric neurodegenerative disorder that occurs due to synucleinopathy, i.e., accumulation and aggregation of $\alpha$-synuclein in the substantia nigra in the CNS and in other neural structures [356]. In the CNS, $\alpha$-synuclein, involved in the regulation of neurotransmissions, is abundantly expressed. Accumulation of insoluble polymers of phosphorylated $\alpha$-synuclein in the perykaria of the neuronal body of substantia nigra may cause neurodegeneration and neuronal death.

A direct association between the gut microbiota and PD has not been clearly established. It is suggested that the gut microbiota increases the intestinal permeability in patients with PD causing the release of $\alpha$-synuclein from the intestine into the circulation and finally to the brain via the vagal nerve [357]. The composition of gut microbiota undergoes alteration during aging. For example, the ratio of Bacteroidetes to Firmicutes increases, whereas the level of bifidobacteria decreases. Moreover, a close association between Prevotellaceae and other bacteria and the motor phenotype of patients with PD has been reported [358].

In patients with PD, a reduced abundance of bacteria belonging to the family Prevotellaceae and increased levels of Enterobacteriaceae were observed in comparison with healthy controls [358]. Bacteria belonging to the family Prevotellaceae are commensals, which are involved in the synthesis of mucin in the gut mucosal barrier. Decreased abundance of Prevotellaceae leads to decreased synthesis of mucin, causing increased intestinal permeability. In patients with PD and animal models of PD, an association of inflammatory changes with increased colonic permeability has been reported [359].

In 54 to $67 \%$ of patients with PD, a high prevalence of small intestinal bacterial overgrowth (SIBO) has been reported [360]. It is suggested that SIBO predisposes to motor function impairment [360] and facilitates the translocation of bacteria and endotoxins across the intestinal epithelium. The induced pro-inflammatory response may cause motor dysfunction by disrupting the integrity of the small intestine, resulting in immune stimulation and/or altered absorption of L-dopa. Another hypothesis postulates changes in gut permeability in comparison with healthy controls. In this manner, SIBO facilitates the translocation of bacteria and endotoxins across the intestinal epithelium, inducing the pro-inflammatory response [361]. In fecal samples of patients with PD, significantly reduced amounts of butyrate-producing bacteria, belonging to the genus Faecalibacterium, Coprococcus, and Roseburia were observed, whereas the increased abundance of Ralstonia was detected in the mucus of these patients [362]. Another study revealed an increased level of Lactobacillus, Bifidobacterium, Akkermansia, and Verrucomicrobiaceae in patients with PD, whereas levels of Faecalibacterium, Coprococcus, Blautia, and Prevotella reduced [363]. The composition of the gut microbiota also correlated with the PD stage. The levels of cellulosedegrading bacteria from genera Blautia, Faecalibacterium, and Ruminococcus significantly decreased in patients with PD, whereas the levels of putative pathobionts belonging to the genera Escherichia-Shigella, Streptococcus, Proteus, and Enterococcus increased in these patients in comparison with healthy controls [364]. Moreover, researchers observed that the disease severity and duration of PD negatively correlated with the cellulose-degrading bacteria and positively correlated with the pathobionts. According to the author's suggestion, observed changes in the gut microbiota may reduce the production of SCFAs and increase the production of endotoxins and neurotoxins, which may cause the development of PD [364]. As a potential triggering factor in the 
pathogenesis of PD, the involvement of $H$. pylori has been suggested. However, its role in the development of PD remains controversial [365]. Infection by $H$. pylori may predispose an individual to autoimmunity, causing damage to neurons, which may lead to the development of parkinsonism [366]. The role of $H$. suis has been suggested in the pathogenesis of PD, a common zoonotic species of Helicobacter in humans. In patients with idiopathic parkinsonism, a higher frequency of this bacterium in comparison with healthy control subjects was observed [367]. It is suggested that infection by viruses, such as H5N1 influenza virus and HCV (not HBV), may cause PD [368, 369].

Modification of the gut microbiota has been suggested as a therapy for the prevention and treatment of PD. These changes could be attributed to the administration of probiotics, prebiotics, or fecal microbiota transplantation [370, 371].

Multiple sclerosis (MS) is a chronic, autoimmune disease, mediated by the invasion of the CNS by immune cells. It is suggested that a link exists between MS and gut microbiota [122]. This autoimmune disease could be attributed to the invasion of the CNS by immune cells. It was found that commensal microbiota is necessary to activate myelin-specific $B$ cells and invade the neurons by effector T cells [372-374].

A specific type of microbiome $[375,376]$ has been reported during dysbiosis of gut microbiota In patients with MS. Patients with MS, in comparison with healthy controls, have a relatively low abundance of bacterial genera, such as Bacteroides, Parabacteroides, Prevotella, Butyricimonas, Faecalibacterium, Eubacterium, Clostridium, Lactobacillus, Coprobacillus, Collinsella, Adlercreutzia, and Slackia, and families, such as Lachnospiraceae, Ruminococceae, Erysipelotrichaceae, and Veillonellaceae. In these patients, increased levels of genera Blautia, Dorea, Streptococcus, Ruminococcus, Acinetobacter, Bifidobacterium, Eggerthella, Pseudomonas, Mycoplasma, Haemophilus, Bilophila, Sutterella, and Akkermansia were observed [377-380]. In the case of the genus Akkermansia, a mucine-degrading bacterium, patients with MS had high levels of $A$. calcoaceticus and A. muciniphila [378]. These bacteria caused the transformation of mucin into SCFAs, and therefore, could compensate the inflammatory state in patients with MS [379].

Oral intake of polysaccharides derived from the human commensal $B$. fragilis exerts a beneficial role. It reduces the experimental autoimmune encephalomyelitis pathologies in animal models of MS, demyelination, and levels of interferon- $\nu$ and IL-17, and increases the levels of IL-10 and conversion of T cells to Tregs [381, 382].

\subsection{Dysbiosis and Lung Diseases}

Lungs have a low density of microbiota $-10^{3}$ to $10^{5}$ colony-forming units/g of the lung tissue [383], which reflects good health.

Most of the bacteria in the lung microbiota belong to four phyla: Bacteroidetes, Firmicutes, Proteobacteria, and Actinobacteria with predomination of two phyla: Bacteroidetes and Firmicutes. In a healthy human, the most abundant genera in the lungs are Prevotella, Streptococcus, Veillonella, Neisseria, Haemophilus, and Fusobacterium [384].

In patients with lung diseases, such as asthma, the composition of microbiota gets altered. However, it is not yet known if asthma is a cause or result of dysbiosis. An analysis of bronchoalveolar lavage in children with asthma revealed different distribution, with, in order of abundance of Proteobacteria, Firmicutes (mainly Streptococcus), Bacteroidetes (mainly Prevotella), and Actinobacteria in comparison with healthy controls [384]. In children with asthma, more 
abundant microbes belonged to the genera Staphylococcus and Haemophilus, whereas Prevotella was found to be more abundant in healthy children [384]. Moreover, it was observed that in patients with asthma, lung microbiota was more diverse and abundant [385]. A fecal transplant from a child at risk for asthma into the GF (Germ-free) mice caused severe lung inflammation after challenge with an allergen, ovalbumin [386]. There is no evidence to support a beneficial role of probiotics used in the perinatal period against asthma or children wheeze [387].

The gut microbiota may play a protective role against lung diseases. For example, the gut microbiota is involved in the host defense against S. pneumoniae infections; however, the precise role of the intestinal microbiota in this process remains unknown [388].

Microbiota in healthy human lungs comprises environmental fungi or those disseminating from the oral cavity. These fungi belong to the genera Cladosporium, Aspergillus, Penicillium, and Candida [389]. Moreover, fungal dysbiosis might cause lung diseases, such as severe asthma [390] and cystic fibrosis [391]. Increased number of mycobiota and reduced $\alpha$-diversity have been observed in the lungs of patients with cystic fibrosis, which correlates with disease severity. In these patients, dominant fungal genera include Candida and Aspergillus, which are linked to morbidity [391]. However, it is unclear whether the effect is local or is due to the intestinal mycobiota [392].

\subsubsection{Dysbiosis and Cancer}

A study suggests that gut microbiota plays a role in the development of cancer. The first such study reported that bacteria could cause stomach cancer after the presence of $H$. pylori in the stomach was discovered [393].

\subsubsection{Cancers of Digestive System}

Oral cavity cancer, primarily oral squamous cell carcinoma (OSCC), arises from the oral mucosa. In a healthy oral cavity, 772 bacterial species reside that belong to 185 genera and 12 phyla [394, 395]. Furthermore, the oral cavity consists of non-bacterial microorganisms such as protozoa and 85 fungal genera [396].

OSCC could result from environmental factors, as well as from alterations in the composition of oral microbiota [395]. Reduced species richness and diversity were observed in samples obtained from OSCC tissues in patients with fibroepithelial polyp (FEP), benign hyperplasia of the oral mucosa, as compared with control subjects. The over-represented genera in these samples included Capnocytophaga, Pseudomonas, and Atopobium, whereas the most abundant genera present in samples obtained from the FEP controls were Lautropia, Staphylococcus, and Propionibacterium [397]. A study reported drastic changes in the composition of bacterial communities of OSCC [395]. Another study revealed significantly higher bacterial diversity in cancer samples in comparison with samples from healthy controls. The oral microbiota of patients with OSCC had a significantly high abundance of Fusobacterium, Peptostreptococcus, Filifactor, Peptococcus, Catonella, and Parvimonas. Poor oral hygiene may cause the overgrowth of pathogenic bacteria. Increased risk of OSCC is associated with the presence of periodontopathogenic bacteria, such as Prevotella tannerae, $P$. intermedia, and Fusobacterium nucleatum, which may induce the inflammation of the squamous oral mucosal epithelium [398]. In comparison with the healthy control group, Peptostreptococcus, Fusobacterium, Alloprevotella, and Capnocytophaga were more abundantly present in samples obtained from Japanese patients with oral cancer, with a lower abundance of Rothia and 
Haemophilus [399]. A strong association between oral cancer and epithelial precursor lesions was observed in the case of bacteria belonging to genera Fusobacterium, Veilonella, Actinomyces, Clostridium, Haemophilus, as well as Enterobacteriaceae [400].

Colonization with fungal species is considered a risk factor in OSCC. In patients with OSCC, a significantly high level of Candida albicans was reported [401].

The pathogenesis of cancer could be attributed to three mechanisms of action of oral microbiota: stimulation of chronic inflammation by bacteria, affecting cell proliferation, cytoskeletal rearrangements, activation of NF-kB, and inhibition of cellular apoptosis; and production of carcinogenic substances [402]. Further research is required to define the exact role of oral bacteria in the development of OSCC.

Esophageal cancer (EC) is the eighth most common cancer; it has two major histological types: esophageal adenocarcinoma (EAC) and esophageal squamous cell carcinoma (ESCC).

Investigations of normal esophageal microbiome revealed the presence of bacteria belonging to six phyla: Firmicutes, Bacteroidetes, Actinobacteria, Proteobacteria, Fusobacteria, and TM7. Moreover, 97 species with the most common genera Streptococcus, Prevotella, and Veillonella were detected [403]. The distal esophagus contains microflora similar to that of the oropharynx; however, the phylum Spirochaetes, prevalent in oral microflora, are absent. Gut dysbiosis is associated with several esophageal diseases; however, it is unclear whether esophageal diseases are due to changes in the gut microbiota or changes in the gut microbiota in the esophageal environment cause changes in the microbial composition [404]. Several investigations suggest the association of gut dysbiosis with several esophageal diseases, such as Barrett's esophagus (BE), gastroesophageal reflux disease (GERD), irritable bowel syndrome, colitis, and esophageal cancer.

Bacteria isolated from patients with BE are closely associated with the mucosa of the specimens. Therefore, the authors conclude that Barrett's mucosa is colonized by resident bacteria [405]. Another investigation showed a greater abundance of gram-negative anaerobes/microaerophiles from phyla Bacteroidetes, Proteobacteria, Fusobacteria, and Spirochaetes in patients with GERD and $B E$ as compared with healthy subjects. The composition of microbiota did not differ between patients with GERD and BE. Therefore, researchers suggest that inflammation and intestinal metaplasia are associated with changes in the microbiome in the distal esophagus [406]. Results obtained from other studies revealed that Veillonella, Prevotella, Neisseria, and Fusobacterium are abundantly present in patients with BE. These bacteria were not detected in the esophagus of healthy subjects, and recently published studies by other authors confirmed these results [404, 407].

A higher abundance of Treponema denticola, Streptococcus mitis, and S. anginosus was found in surgically resected EAC in comparison to healthy control tissue [408]. Another research showed decreased diversity in patients with EAC as compared with healthy controls and patients with BE. The reduced genera included Veillonella and Granulicatella, whereas an abundance of L. fermentum was found to be enriched in patients with EAC [409]. Moreover, a study reports a well-documented inverse correlation between the risk of EAC and chronic infection with H. pylori [410]. H. pylori may affect carcinogenesis in the lower esophagus; however, the mechanisms of this dependence has remained unclear, as well as the association between the microbiome and esophageal adenocarcinoma.

The data available regarding the microbiome in patients with ESCC are minimal, and the microbiome in ESCC is less well characterized than in EAC. It was found that gastric corpus microbiota affected by ESCC was enriched in Clostridiales and Erysipelotrichales [411]. 
Porphyromonas gingivalis was detected in the esophageal mucosa of patients with ESCC, but not the mucosa of healthy controls. The presence of these bacteria was positively correlated with cancer cell differentiation and metastasis and with poor clinical outcome [412]. Changes in the composition of bacterial microbiota in the saliva is related to a higher risk of ESCC. In patients with ESCC, a decreased abundance of genera Lautropia, Bulleidia, Catonella, Corynebacterium, Moryella, Peptococcus, and Cardiobacterium was detected in comparison to healthy controls [413]. Results obtained in other studies revealed a higher proportion of Firmicutes and a lower proportion of Gammaproteobacteria and higher levels of Bacilli in patients with ESCC than in healthy control groups. Moreover, slightly lower $\alpha$-diversity and richness were detected in these patients in comparison to healthy controls. In contrast, $\beta$-diversity was higher than that in healthy subjects [414]424]. An important role in the development of ESCC is played by F. nucleatum, which primarily inhabits the oral cavity and is frequently detected in colon cancer tissue. In about $23 \%$ of patients, who underwent surgical resection of esophageal cancer, $F$. nucleatum was detected in cancer tissues. The presence of these bacteria in cancer tissue was also associated with a significantly shorter survival time [414].

Gastric cancer (GC) is the fifth most common cancer in the world and the third leading cause of cancer-associated deaths worldwide. The most well-established risk factor for the development of gastric cancer is $H$. pylori infection [415-417]. This bacterium is associated with more than $90 \%$ of GC cases $[417,418]$. Since $1994, H$. pylori has been recognized by the World Health Organization as a "definite carcinogen" $[417,419]$. Specific host responses to $H$. pylori infection include vacuolating cytotoxin (VacA) and cytotoxin-associated antigen A (CAGA). Moreover, it is associated with a higher risk of gastric cancer [420]. VacA stimulates the apoptosis of gastric epithelial cells, suppresses the immune responses by stimulation of dendritic cells to release the anti-inflammatory cytokines (IL10 and IL-18), promotes $H$. pylori evasion, and enhances tumor survival [421]. Some strains of $H$. pylori consist of the cag pathogenicity island (PAI), which increases the risk of adenocarcinoma of the stomach [422]. The cag PAI has genes that encode specific proteins, which form the bacterial type IV secretion system. This system exports CagA and peptidoglycans from bacteria into the host cells to activate the PI3-K pathway and contribute to carcinogenesis [423].

Gastric acidity influences the composition of the microbiome in the human stomach [424] . Acidic environment renders the human stomach as not amenable for colonization by bacteria other than $H$. pylori. The bacteria stimulate the secretion of gastrin, leading to more gastric acid production and making patients more vulnerable to duodenal ulcers. In contrast, it protects patients against GC [425]. H. pylori may suppress the production of gastric acid through inflammatory mediators, causing progressive loss of gastric glands and leading to atrophic gastritis [426]. Reduced secretion of gastric acid increases the gastric $\mathrm{pH}$, can encourage colonization and proliferation of bacterial species other than $H$. pylori from the oral cavity, the upper respiratory tract, or the intestine. These changes disrupt the microbial balance of the gastric mucosal ecological niche, resulting in an increased nitrosating species and raised nitride and $\mathrm{N}$-nitrosamine levels in the stomach.

Several findings have suggested the potential involvement of microbes other than $H$. pylori in the carcinogenesis of the stomach. The examination of gastric mucosal microbiota in patients with $H$. pylori-negative gastritis $(\mathrm{HpN})$ and $H$. pylori-positive gastritis, and $H$. pylori-negative non-gastritis group as a control revealed significantly lower bacterial richness in the HpP patients. In contrast, enrichment of Firmicutes, Fusobacteria, Bacteroidetes, and Actinobacteria was detected at the phylum level in the HpN group. It was found that Streptococcus sp. and Haemophilus parainfluenzae 
significantly increased the risk for $\mathrm{HpN}$. The presence of Treponema sp. was unique to $\mathrm{HpN}$ patients. Therefore, Streptococcus sp., H. parainfluenzae, and Treponema sp. should be treated as potential pathogenic bacterial species for HpN [427]. Patients with GC after the surgical procedure showed a higher diversity of species and richness as compared with healthy control subjects. In these patients, a higher abundance of aerobes, facultative anaerobes, and oral microbes was observed. The levels of $F$. nucleatum, a colorectal cancer-related bacterium, were significantly higher in patients who underwent total gastrectomy compared with the control samples [428]. In cancer samples, oral bacteria, such as Peptostreptococcus, Streptococcus, and Fusobacterium were predominantly detected. In the non-tumor tissues, lactic acid-producing bacteria, such as Lactobacillus lactis and $L$. brevis were present in more abundance. At the phylum level, the predominant phylum in the cancerous samples was Proteobacteria (overall $90 \%$ of cancerous samples), followed by Firmicutes, Bacteroidetes, Actinobacteria, Acidobacteria, and Fusobacteria. In non-cancerous tissues, Proteobacteria (higher levels in comparison with cancerous samples) predominated and, in comparison with cancerous tissues, decreased levels of Firmicutes, Bacteroidetes, Actinobacteria, and Fusobacteria were found [429]. Bacteria such as E. coli, Lactobacillus, Nitrospirae, Clostridium, Veillonella, Haemophilus, and Staphylococcus that can produce N-nitroso compounds are also present. In contrast, Streptococcus, Prevotella, and Neisseria are associated with a low risk of GC development $[417,430]$. According to authors, colonization by $H$. pylori changes the composition of the microbiota, and these changes, especially of oral microbiota, may influence the maintenance of the local microenvironment, resulting in the development or progression of GC. To date, the role of the gastric microbiome in the development of GC has remained largely unclear [431]. In contrast, $H$. pylori eradication therapy is suggested as effective in preventing GC [432].

Colorectal cancer (CRC) is one of the most diagnosed malignancies. Obtained results suggest the role of the intestinal microbiota in the development of colorectal cancer. It was observed that enterotoxigenic $B$. fragilis produces a toxin (fragilysin) that stimulates cell proliferation and induces the synthesis of inflammatory mediators [433]. In animal studies, this human colonic commensal bacterium was shown to increase the risk of development of colon adenomas and tumors [434]. In contrast, several bacterial metabolites suppress the development of colon cancer. These include SCFAs (acetate, propionate, and butyrate). Especially, butyrate protects against colonic neoplasia. Of note, whether the dysbiosis is a cause or consequence of adenomas and CRC is still unclear [435].

Several species of bacteria garner attention for their associations with CRC. The primary risk factor in up to $80 \%$ of all cases of CRC is diet, and a strong association between the diet and composition of gut microbiota with the risk of developing CRC has been observed [436]. The development of CRC could be attributed to microbial dysbiosis[437]. Investigation of fecal samples of patients with $\mathrm{CRC}$ revealed higher levels of bacteria belonging to the group Bacteroides-Prevotella in comparison with healthy controls [438]. In the gut microbiota of patients with CRC, increased prevalence of bacteria belonging to Enterococcus, Escherichia, Shigella, Klebsiella, Streptococcus, Peptostreptococcus, Pseudomonas, Helicobacter, Acinetobacter was found, whereas the prevalence of bacteria belonging to Lachnospiraceae, as well as to genera Roseburia, Clostridium, Faecalibacterium, and Bifidobacterium, butyrate-producing bacteria was low [439, 440]. Other investigations and meta-analyses showed that microbiota from patients with CRC contained a higher proportion of Fusobacterium, Parvimonas, Butyrivibrio, Gemella, Fusobacteria, and Akkermansia. Microbiome is often enriched in proinflammatory opportunistic pathogens and microbes that cause metabolic disorders, such as Streptococcus bovis, F. nucleatum, E. coli, B. fragilis, 
and Enterococcus faecalis, and lower proportions of Ruminococcus, Bifidobacterium, Eubacteria, and Lachnospira in comparison with healthy controls [441-443]. The higher abundance of Bacteroides and bifidobacteria increases the risk of colon polyps, whereas that of Lactobacillus and Eubacterium is protective [444]. A study reported an association of increased risk of colon cancer with a higher abundance of bacteria producing hydrogen sulfide and bile salts [445]. Several other studies describe the association between gut microbial dysbiosis and colorectal cancer.

Different hypotheses have been introduced to explain the role of microbial dysbiosis in carcinogenesis. It was suggested the epigenetic nature for host DNA alterations and the association between gut microbial dysbiosis and colorectal cancer caused gene methylation. This suggestion was confirmed in animal and human studies; therefore, researchers conclude that dysbiosis associated with $\mathrm{CRC}$, inducing methylation of host genes, may promote carcinogenesis via dysregulation of the genome [437].

An increased abundance of $F$. nucleatum was observed in patients with CRC. It was found that $F$. nucleatum surface protein, FadA binds to E-cadherin on epithelial cells, activating the oncogenic, pro-proliferative $\beta$-cadherin signaling [446]]. Moreover, $F$. nucleatum can alter the functions of tumor-infiltrating lymphocytes and natural killer (NK) cells [447]. This bacterium also stimulates the production of inflammatory cytokines such as IL-6, IL-8, and IL-18[446]. Moreover, a correlation was found between the levels of $F$. nucleatum with tumor invasion, lymph nodes, and distal metastasis [448].

Bacteroides fragilis is an enterotoxigenic (ETBF) bacterium that may play a role in colorectal carcinogenesis. As mentioned earlier, this bacterium produces toxin called $B$. fragilis-derived toxin (BFT). It activates the host's spermine oxidase, generating hydrogen peroxide and reactive oxygen species (ROS), which initiate DNA damage in epithelial cells and promote tumorigenesis [449]. BFT interacts with the host's epithelial E-cadherin to disrupt the intracellular junction, activate $\beta$-catenin nuclear signaling, thus inducing cellular proliferation. B. fragilis may activate Th17 immune responses and may lower the host's anti-tumor immune responses, resulting in unhindered growth of cancer cells [434, 450]. Animal studies have demonstrated that $B$. fragilis exacerbates tumorigenesis in susceptible mice [434]. Adenomatous polyposis coli (APC) null mouse is used as a model of colon cancer. In the $\mathrm{APC}^{\mathrm{min} /+}$ mice, B. fragilis induced tumorigenesis through an inflammation-dependent mechanism [434] involving activation of Th17 via Stat3 [434].

E. coli has a higher prevalence in patients with CRC in comparison with the healthy controls [441]. This enterotoxic bacterium expresses the genomic island polyketide synthase $\left(\mathrm{pks}^{+}\right)$. This gene enhances tumorigenesis in animal models of colorectal cancer and is enriched in human tissues. In colon tissue samples, the toxigenic pks gene cluster was found in the metagenome in 14 of 21 patients (66.6\%) with CRC and only in 5 of $24(20.8 \%)$ healthy control subjects [451]. E. coli pks ${ }^{+}$ produces a genotoxin termed colibactin [451], which alkylates DNA, resulting in double-strand breaks in mammalian cells, such as colonic epithelial cells [452]. Moreover, it triggers premature and transmissible cellular senescence in cells, which initially survive the DNA damage [453]. Feeding animal models of CRC with $E$. coli containing the $p k s$ gene resulted in increased cases of tumors in these mice [451].

Pancreatic ductal adenocarcinoma (PDAC) is a lethal and the most aggressive and devastating malignancy.

Growing evidence suggests that gut microbiota might influence pancreatic carcinogenesis, and is associated with PDAC susceptibility, initiation, and progression [454]. Moreover, the gut microbiota 
could influence therapeutic efficacy; however, the role of the microbiota in pancreatic carcinogenesis remains unclear.

The pancreas was traditionally considered a sterile organ due to the presence of numerous proteases and a highly alkaline environment [455]. In cancerous pancreatic tissues, increased bacterial abundance was observed; for example, a 1000-fold increase in intrapancreatic bacteria in patients with PDAC was observed as compared with normal pancreatic tissue [456]. The presence of Fusobacterium sp. in the pancreatic tissues correlated with poor pancreatic cancer prognosis [457]. The presence of Gammaproteobacteria in PDAC tissue specimens reduced the therapeutic effects by metabolizing the anticancer drug, gemcitabine [458]. H. pylori can colonize the pancreas and may initiate the process of carcinogenesis; however, different subspecies of Helicobacter have been identified in the pancreas [459]. Several investigations suggest the role of $H$. pylori in the development of PDAC. Pathogenic components derived from $\mathrm{H}$. pylori, such as ammonia, LPS, and inflammatory cytokines, damage the pancreas. H. pylori infection activates NF-KB and the activator protein-1 (AP-1), dysregulating cellular processes. LPS from these bacteria may cause mutations in $K$-Ras gene, which are detected in over $90 \%$ of the cases of PDAC [460]. This bacterium activates the signal transducer and activator of transcription 3 (STAT3), causing the overexpression of antiapoptotic and pro-proliferative proteins such as Bcl-xL, MCL-1, survivin, c-myc, and cyclin D1. Changes in the expression of these proteins may promote the progression of pancreatic cancer [461].

Pancreatic carcinogenesis and increased risk of PDAC may also be caused by oral microbiota, periodontal diseases, and tooth loss $[459,462]$. Dysbiosis of oral microbiota preceded the development of pancreatic cancer rather than developed PDAC and stimulated microbiota dysbiosis. In blood samples of patients with PDAC, a significantly higher level of antibodies against $P$. gingivalis, a periodontal pathogen, which causes chronic periodontitis, as compared to healthy controls, was observed. The highest abundance of $P$. gingivalis was associated with a twofold increased risk of pancreatic carcinogenesis [463]. The presence of $P$. gingivalis and Aggregatibacter actinomycetemcomitans in the oral cavity increases the risk of pancreatic cancer [464], whereas the presence of Fusobacteria and Leptotrichia decreases this risk [465]. Investigations of salivary samples showed that patients with pancreatic cancer had higher levels of Leptotrichia and Bacteroides, whereas levels of $P$. gingivalis, Neisseria elongata, and A. actinomycetemcomitans, were lower. The main oral bacteria involved in PDAC carcinogenesis are $P$. gingivalis, Fusobacterium, N. elongata, and Streptococcus mitis [459]. Three species of bacteria, P. gingivalis, T. denticola, and Tannerella forsythia, known as "the red complex" are described as the major pathogens causing periodontitis. These bacteria secrete peptidyl-arginine deiminase (PAD) enzyme and may cause point mutations in $p 53$ (tumor suppressor gene) and oncogene $K$-Ras. It was found that $p 53$ Arg72Pro and $K$-Ras codon 12 arginine mutations indicated poor prognosis of patients with PDAC [466]. Fusobacterium spp. are pathogens that cause periodontal diseases. This bacterium increases the production of reactive oxygen species (ROS), inflammatory cytokines, and modulates the tumor microenvironment. Therefore, it potentiates tumorigenesis [446]. In contrast, results from other studies suggest an association of Fusobacterium with reduced risk of PDAC [465]. Investigations of saliva samples of patients with PDAC showed higher levels of Leptotrichia, Bacteroides, Granulicatella adiacens, Porphyromonas, Streptococcus, Prevotella, Campylobacter, Atopobium, and Neisseria [464], whereas N. elongata, S. nitis, Corynebacterium, and Aggregatibacter were found to be significantly lower in patients with PDAC as compared with healthy controls[464]. However, a report suggests that $A$. actinomycetemcomitans is linked to a higher risk of development 
of PDAC [465]. It is suggested that Leptotricha plays a protective role and decreases the risk of PDAC. However, a higher ratio of Leptotrichia to Porphyromonas in saliva samples was detected in patients with PDAC.

Investigations of fecal samples from patients with newly diagnosed pancreatic cancer revealed a higher abundance of Bacteroides, Verrucomicrobia, Sutterella, Veillonella, Odoribacter, and Akkermansia, and lower levels of Firmicutes and Actinobacteria, as compared with healthy subjects [467]. In another study, selective enrichment of Proteobacteria, Synergistetes, and Euryarchaeota in fecal samples of patients with PDAC was observed [468]. Comparison of microbial composition in surgically resected patients who survived more than five years after surgery (long-term survival [LTS], median survival of 10.1 years) with patients who survived less than five years (short-time survival [STS], median survival of 1.6 years) revealed a higher $\alpha$-diversity in the tumor microbiome composition in patients with LTS than in patients with STS [469]. Moreover, researchers have observed a higher abundance of Pseudoxanthomonas, Saccharopolyspora, and Streptomyces with LTS as compared with STS. The authors conclude that the presence and abundance of these three genera along with the presence of Bacillus clausii, influence the prediction of LTS in patients with PDAC [469]. Fecal microbiota transplantation from STS patients or LTS patients into mice can modulate the tumor microbiome and affect the tumor growth and tumor immune infiltration [469].

There are also investigations that describe oral microbiota in patients with intraductal papillary mucinous neoplasms (IPMNs), which are most common among pancreatic cystic neoplasms (PCNs). PCNs are characterized by malignant transformation to invasive carcinoma. It is suggested that IPMN could be considered an early detection marker of pancreatic cancer [462]. IPMNs display varying degrees of cellular dysplasia, from low-grade dysplasia through high-grade dysplasia to invasive carcinoma. In samples from patients with IPMNs, a higher abundance was observed in the case of F. nucleatum and G. adiacens in comparison with healthy controls [462]. In another study, IPMN high-grade dysplasia was found to be highly enriched for genera Granulicatella, Serratia, and Fusobacterium, whereas low levels of Methylobacterium, Sphingomonas, and Propionibacterium were observed in comparison to remainder two diagnosis groups [462].

As described above, microbiota induces PDAC via multiple pathways $[459,469,470]$.

Hepatocellular carcinoma (HCC) and cholangiocarcinoma (CCA) are the most common histological types of liver cancer. There is little evidence on the associations between the gut microbiota and HCC in humans.

In fecal samples of patients with HCC, higher counts of $E$. coli were detected compared with healthy controls $[471,472]$. Another research revealed reduced numbers of Bifidobacterium and enrichment of Bacteroides and Ruminococcus in these patients as compared with healthy controls [473]. In patients with early HCC, increased levels of Actinobacteria phylum and 13 genera including Gemmiger, Parabacteroides, Prevotella, Alistipes, Phascolarctobacterium, and Ruminococcus were observed, whereas reduced numbers of Verrucomicrobia, Klebsiella, and Haemophilus were observed in comparison with healthy controls. Butyrate-producing bacteria were decreased, whereas genera producing LPS increased in patients with HCC. In early HCC, increased gut microbial diversity was observed [474]. The role of Helicobacter spp. in the development of HCC has been reported. Helicobacter spp. have been detected in the liver of patients with primary hepatic carcinoma, whereas this bacterium is not present in healthy controls [475]. H. hepaticus is not present in HCC patients with chronic hepatitis B or C. H. pylori is detected in HCC patients with HCVhepatitis [476]. Moreover, it was found that other Helicobacter species, such as H. pylori, H. bilis, $H$. 
hepaticus, and $H$. ganmani are specifically associated with cholangiocarcinoma, but not with nontumor diseases in the bile duct [477], and Salmonella typhi is associated with gallbladder and hepatobiliary carcinoma [478]. Another study described changes in the composition of the tongue coating in patients with HCC.

LPS produced by bacteria can activate TLR4, resulting in the development of liver cancer. In a mouse model of HCC, LPS influenced hepatic Kupffer cells that produce inflammatory cytokines, such as TNF- $\alpha$ and IL-6. Paracrine activated the cytokines through the LPS-TLR4-NF-KB signaling pathway to stimulate precancerous hepatocellular proliferation and induce HCC [479]. Bile acids (BAs) are common and important components of the gastrointestinal tract. Gut microbiota influences liver cancer pathogenesis through BA-mediated regulation of the liver immune system. For example, secondary BAs suppress anti-tumor immunity, promoting liver cancer progression. This suppression could be attributed to the prostaglandin E receptor on CD8 T cells [480]. Further, liver cancer can be regulated by NKT cells. The inhibition of Clostridium species enhances the accumulation of NKT cells in the liver, whereas colonization of the gut by $C$. scindens decreases the number of NKT cells in the liver. Clostridium spp. are responsible for the enzymatic conversion of primary BAs to secondary BAs.

Associations between gut microbiota dysbiosis and the development of $\mathrm{HCC}$ were also described in other studies [473, 481, 482].

Dysbiosis of microbiota may result in cancers such as lung cancer (mainly due to dysbiosis of airway and lung microbiota) [483], melanoma (due to dysbiosis of skin microbiota) [484], cancers of the urogenital system (due to dysbiosis of urinary tract microbiota) $[485,486]$, and breast cancer (due to dysbiosis of breast tissue microbiota) [487].

\section{Concluding Remarks}

Gut microbiota plays an important role in human health and diseases by affecting the host metabolism and immune function. Changes in the composition of gut microbiota, termed dysbiosis, may cause several diseases. In contrast, alterations in the composition of these bacteria may have a beneficial role. Probiotic bacteria are often consumed as part of foods such as yogurts and cheese, as well as drugs, in the case of antibiotic therapy. Commensal bacteria inhibit the colonization by pathogens, protect against physiological stress, and stimulate the immune system. Therefore, further investigation into the relationship between gut microbiota and human health, as well as between dysbiosis and diseases, is warranted.

\section{Funding}

This research did not receive any specific grant from funding agencies in the public, commercial, or not-for-profit sectors.

\section{Competing Interest}

Authors declare no competing of interest.

\section{References}


1. Muñoz-Garach A, Diaz-Perdigones C, Tinahones FJ. Gut microbiota and type 2 diabetes mellitus. Endocrinol Nutr. 2016; 63: 560-568.

2. Zhang X, Qi Q, Zhang C, Smith SR, Hu FB, Sacks FM, et al. FTO genotype and 2-year change in body composition and fat distribution in response to weight-loss diets: The pounds lost trial. Diabetes. 2012; 61: 3005-3011.

3. Chen Z, Zhu S, Xu G. Targeting gut microbiota: A potential promising therapy for diabetic kidney disease. Am J Transl Res. 2016; 8: 4009-4016.

4. Moreno-Indias I, Cardona F, Tinahones FJ, Queipo-Ortuno MI. Impact of the gut microbiota on the development of obesity and type 2 diabetes mellitus. Front Microbiol. 2014; 5: 190.

5. Ley RE, Peterson DA, Gordon JI. Ecological and evolutionary forces shaping microbial diversity in the human intestine. Cell. 2006; 124: 837-848.

6. Dominguez-Bello MG, Costello EK, Contreras M, Magris M, Hidalgo G, Fierer N, et al. Delivery mode shapes the acquisition and structure of the initial microbiota across multiple body habitats in newborns. Proc Natl Acad Sci U S A. 2010; 107: 11971-11975.

7. Zoetendal EG, Rajilic-Stojanovic M, De Vos WM. High-through put diversity and functionality analysis of the gastrointestinal tract microbiota. Gut. 2008; 57: 1605-1615.

8. Wu GD, Chen J, Hoffmann C, Bittinger K, Chen YY, Keilbaugh SA, et al. Linking long-term dietary patterns with gut microbial enterotypes. Science. 2011; 334: 105-108.

9. Meyer TW, Hostetter TH. Uremic solutes from colon microbes. Kidney Int. 2012; 81: 949-954.

10. Fallucca F, Porrata C, Fallucca S, Pianesi M. Influence of diet on gut microbiota, inflammation and type 2 diabetes mellitus. First experience with macrobiotic Ma-Pi 2 diet. Diabetes Metab Res Rev. 2014; 30 Suppl 1: 48-54.

11. Wong JM, de Souza R, Kendall CW, Emam A, Jenkins DJ. Colonic health: Fermentation and short chain fatty acids. J Clin Gastroenterol. 2006; 40: 235-243.

12. De Vadder F, Kovatcheva-Datchary P, Goncalves D, Vinera J, Zitoun C, Duchampt A, et al. Microbiota-generated metabolites promote metabolic benefits via gut-brain neural circuits. Cell. 2014; 156: 84-96.

13. Liou AP, Paziuk M, Luevano JM Jr., Machineni S, Turnbaugh PJ, Kaplan LM. Conserved shifts in the gut microbiota due to gastric bypass reduce host weight and adiposity. Sci Transl Med. 2013; 5: 178 ra141.

14. Sonnenburg JL, Backhed F. Diet-microbiota interactions as moderators of human metabolism. Nature. 2016; 535: 56-64.

15. Donohoe DR, Garge N, Zhang X, Sun W, O'Connell TM, Bunger MK, et al. The microbiome and butyrate regulate energy metabolism and autophagy in the mammalian colon. Cell Metab. 2011; 13: 517-526.

16. Donohoe DR, Wali A, Brylawski BP, Bultman SJ. Microbial regulation of glucose metabolism and cell-cycle progression in mammalian colonocytes. PloS one. 2012; 7: e46589.

17. Wichmann A, Allahyar A, Greiner TU, Plovier H, Lunden GO, Larsson T, et al. Microbial modulation of energy availability in the colon regulates intestinal transit. Cell Host Microbe. 2013; 14: 582-590.

18. Davie JR. Inhibition of histone deacetylase activity by butyrate. J Nutr. 2003; 133: 2485S-2493S.

19. Thorburn AN, McKenzie C, Shen S, Stanley D, Macia L, Mason $L$, et al. Evidence that asthma is a developmental origin disease influenced by maternal diet and bacterial metabolites. Nat Commun. 2015; 6: 7320. 
20. Russell DW. The enzymes, regulation, and genetics of bile acid synthesis. Annu Rev Biochem. 2003; 72: 137-174.

21. Oliphant K, Allen-Vercoe E. Macronutrient metabolism by the human gut microbiome: Major fermentation by-products and their impact on host health. Microbiome. 2019; 7: 91.

22. Granado-Serrano AB, Martin-Gari M, Sanchez V, Riart Solans M, Berdun R, Ludwig IA, et al. Faecal bacterial and short-chain fatty acids signature in hypercholesterolemia. Sci Rep. 2019; 9: 1772.

23. Thomas C, Gioiello A, Noriega L, Strehle A, Oury J, Rizzo G, et al. TGR5-mediated bile acid sensing controls glucose homeostasis. Cell Metab. 2009; 10: 167-177.

24. Heinken A, Ravcheev DA, Baldini F, Heirendt L, Fleming RM, Thiele I. Systematic assessment of secondary bile acid metabolism in gut microbes reveals distinct metabolic capabilities in inflammatory bowel disease. Microbiome. 2019; 7: 75.

25. Thomas C, Pellicciari R, Pruzanski M, Auwerx J, Schoonjans K. Targeting bile-acid signaling for metabolic diseases. Nat Rev Drug Discov. 2008; 7: 678-693.

26. Kawamata $Y$, Fujii R, Hosoya M, Harada M, Yoshida H, Miwa M, et al. A G protein-coupled receptor responsive to bile acids. J Biol Chem. 2003; 278: 9435-9440.

27. Sayin SI, Wahlstrom A, Felin J, Jantti S, Marschall HU, Bamberg K, et al. Gut microbiota regulates bile acid metabolism by reducing the levels of tauro-beta-muricholic acid, a naturally occurring FXR antagonist. Cell Metab. 2013; 17: 225-235.

28. Munro N. Gut microbiota: Its role in diabetes and obesity. Diab Prim Care. 2016; 18: 1-7.

29. Samuel BS, Shaito A, Motoike T, Rey FE, Backhed F, Manchester JK, et al. Effects of the gut microbiota on host adiposity are modulated by the short-chain fatty-acid binding $\mathrm{G}$ proteincoupled receptor, Gpr41. Proc Natl Acad Sci U S A. 2008; 105: 16767-16772.

30. Round JL, Mazmanian SK. The gut microbiota shapes intestinal immune responses during health and disease. Nat Rev Immunol. 2009; 9: 313-323.

31. Atarashi K, Tanoue $T$, Shima T, Imaoka A, Kuwahara $T$, Momose $\mathrm{Y}$, et al. Induction of colonic regulatory T cells by indigenous Clostridium species. Science. 2011; 331: 337-341.

32. Salminen S, Bouley C, Boutron-Ruault MC, Cummings JH, Franck A, Gibson GR, et al. Functional food science and gastrointestinal physiology and function. Br J Nutr. 1998; 80 Suppl 1: S147171.

33. Brahe LK, Astrup A, Larsen IH. Is butyrate the link between diet, intestinal microbiota and obesity-related metabolic diseases? Obesity reviews: An official journal of the International. Obes Rev. 2013; 14: 950-959.

34. Tolhurst G, Heffron H, Lam YS, Parker HE, Habib AM, Diakogiannaki E, et al. Short-chain fatty acids stimulate glucagon-like peptide-1 secretion via the G-protein-coupled receptor FFAR2. Diabetes. 2012; 61: 364-371.

35. Rahim MB, Chilloux J, Martinez-Gili L, Neves AL, Myridakis A, Gooderham N, et al. Diet-induced metabolic changes of the human gut microbiome: Importance of short-chain fatty acids, methylamines and indoles. Acta Diabetol. 2019; 56: 493-500.

36. Nadal I, Donant E, Ribes-Koninckx C, Calabuig M, Sanz Y. Imbalance in the composition of the duodenal microbiota of children with coeliac disease. J Med Microbiol. 2007; 56: 1669-1674.

37. Macfarlane S, Steed H, Macfarlane GT. Intestinal bacteria and inflammatory bowel disease. Crit Rev Clin Lab Sci. 2009; 46: 25-54. 
38. Sjogren YM, Jenmalm MC, Bottcher MF, Bjorksten B, Sverremark-Ekstrom E. Altered early infant gut microbiota in children developing allergy up to 5 years of age. Clin Exp Allergy. 2009; 39: 518-526.

39. Larsen N, Vogensen FK, van den Berg FW, Nielsen DS, Andreasen AS, Pedersen BK, et al. Gut microbiota in human adults with type 2 diabetes differs from non-diabetic adults. PloS one. 2010; 5: e9085.

40. Wu X, Ma C, Han L, Nawaz M, Gao F, Zhang X. Molecular characterization of the faecal microbiota in patients with type 2 diabetes. Curr Microbiol. 2010; 61: 69-87.

41. Westman EC, Feinman RD, Mavropoulos JC, Vernon MC, Volek JS, Wortman JA, et al. Lowcarbohydrate nutrition and metabolism. Am J Clin Nutr. 2007; 86: 276-284.

42. Eaton SB. The ancestral human diet: What was it and should it be a paradigm for contemporary nutrition? Proc Nutr Soc. 2006; 65: 1-6.

43. Dedoussis GV, Kaliora AC, Panagiotakos DB. Genes, diet and type 2 diabetes mellitus: A review. Rev Diabet Stud. 2007; 4: 13-24.

44. Million M, Maraninchi M, Henry M, Armougom F, Richet $H$, Carrier $P$, et al. Obesity-associated gut microbiota is enriched in Lactobacillus reuteri and depleted in Bifidobacterium animalis and Methanobrevibacter smithii. Int J Obes. 2012; 36: 817-825.

45. Alcock J, Lin HC. Fatty acids from diet and microbiota regulate energy metabolism. F1000Res. 2015; $4: 738$.

46. De Filippo C, Cavalieri D, Di Paola M, Ramazzotti M, Poullet JB, Massart S, et al. Impact of diet in shaping gut microbiota revealed by a comparative study in children from Europe and rural Africa. Proc Natl Acad Sci U S A. 2010; 107: 14691-14696.

47. Turnbaugh PJ, Ridaura VK, Faith JJ, Rey FE, Knight R, Gordon JI. The effect of diet on the human gut microbiome: A metagenomic analysis in humanized gnotobiotic mice. Sci Transl Med. 2009; 1: 6 ra14.

48. Santacruz A, Marchos A, Warnberg J, Marti A, Martin-Matillas M, Compoy C, et al. Interplay between weight loss and gut microbiota composition in overweight adolescent. Obesity (Silver Spring). 2009; 17: 1906-1920.

49. Walker AW, Ince J, Duncan SH, Webster LM, Flint HJ. Dominant and diet-responsive groups of bacteria within the human colonic microbiota. ISME J. 2011; 5: 220-230.

50. Backhed F, Ley RE, Sonnenburg JL, Peterson DA, Gordon JI. Host-bacterial mutualism in the human intestine. Science. 2005; 307: 1915-1920.

51. Claesson MJ, Jeffery IB, Conde S, Power SE, O'Connor EM, Cusack S, et al. Gut microbiota composition correlates with diet and health in the elderly. Nature. 2012; 488: 178-184.

52. Ley RE, Backhed F, Turnbaugh P, Lozupone CA, Knight RD, Gordon JI. Obesity alters gut microbial ecology. Proc Natl Acad Sci U S A. 2005; 102: 11070-11075.

53. Oriach CS, Robertson RC, Stanton C, Cryan JF, Dinan TG. Food for thought: The role of nutrition in the microbiota-gut-brain axis. Clin Nutr. 2016; 6: 25-38.

54. Marlow G, Ellett S, Ferguson IR, Zhu S, Karunasinghe N, Jesuthasan AC, et al. Transcriptomics to study the effect of a Mediterranean-inspired diet on inflammation in Crohn's disease patients. Hum Genomics. 2013; 7: 24.

55. De Filippis F, Pellegrini N, Vannini L, Jeffery IB, La Storia A, Laghi L, et al. High-level adherence to a Mediterranean diet beneficially impacts the gut microbiota and associated metabolome. Gut. 2016; 65: 1812-1821. 
56. Ferrocino I, Di Cagno R, De Angelis M, Turroni S, Vannini L, Bancalari E, et al. Fecal Microbiota in Healthy Subjects Following Omnivore, Vegetarian and Vegan Diets: Culturable Populations and rRNA DGGE Profiling. PloS one. 2015; 10: e0128669.

57. Matijai BB, Obermajer T, Lipoglavek L, Grabnar I, Rogelj I. Association of dietary type with fecal microbiota in vegetarians and omnivores in Slovenia. Eur J Nutr. 2014; 53-60.

58. Martínez I, Lattimer JM, Hubach KL, Case JA, Yang J, Weber CG, et al. Gut microbiome composition is linked to whole grain-induced immunological improvements. ISME J. 2013; 7: 269-280.

59. Walsh CJ, Guinane CM, O'Toole PW, Cotter PD. Beneficial modulation of the gut microbiota. FEBS Lett. 2014; 588: 4120-4130.

60. Carvalho-Wells AL, Helmolz K, Nodet C, Molzer C, Leonard C, McKevith B, et al. Determination of the in vivo prebiotic potential of a maize-based whole grain breakfast cereal: $A$ human feeding study. Br J Nutr. 2010; 104: 1353-1356.

61. David LA, Maurice CF, Carmody RN, Gootenberg DB, Button JE, Wolfe BE, et al. Diet rapidly and reproducibly alters the human gut microbiome. Nature. 2014; 505: 559-563.

62. Zhang C, Li S, Yang L, Huang P, Li W, Wang S, et al. Structural modulation of gut microbiota in life-long calorie-restricted mice. Nat Commun. 2013; 4: 2163.

63. Jernberg C, Lofmark S, Edlund C, Jansson JK. Long-term impacts of antibiotic exposure on the human intestinal microbiota. Microbiology. 2010; 156: 3216-3223.

64. Dethlefsen L, Relman DA. Incomplete recovery and individualized responses of the human distal gut microbiota to repeated antibiotic perturbation. Proc Natl Acad Sci U S A. 2011; 108 Suppl 1: 4554-4561.

65. Penders J, Thijs C, Vink C, Stelma FF, Snijders B, Kummeling I, et al. Factors influencing the composition of the intestinal microbiota in early infancy. Pediatrics. 2006; 118: 511-521.

66. Murphy EF, Cotter PD, Hogan A, O'Sullivan O, Joyce A, Fouhy F, et al. Divergent metabolic outcomes arising from targeted manipulation of the gut microbiota in diet-induced obesity. Gut. 2013; 62: 220-226.

67. Dethlefsen L, Huse S, Sogin ML, Relman DA. The pervasive effects of an antibiotic on the human gut microbiota, as revealed by deep 16 S rRNA sequencing. PLoS Biol. 2008; 6: 280-285.

68. McFarland LV. Antibiotic-associated diarrhea: Epidemiology, trends and treatment. Future Microbiol. 2008; 3: 563-578.

69. Anders H, Henrik S. Antibiotic use and intussusception in early childhood. J Antimicrob Chemother. 2009: 642-652.

70. Iapichino G, Callegari ML, Marzorati S, Cigada M, Corbella D, Ferrari S, et al. Impact of antibiotics on the gut microbiota of critically ill patients. J Med Microbiol. 2008; 57: 1007-1014.

71. Forslund K, Hildebrand F, Nielsen T, Falony G, Le Chatelier E, Sunagawa S, et al. Disentangling type 2 diabetes and metformin treatment signatures in the human gut microbiota. Nature. 2015; 528: 262-266.

72. Wu H, Esteve E, Tremaroli V, Khan MT, Caesar R, Manneras-Holm L, et al. Metformin alters the gut microbiome of individuals with treatment-naive type 2 diabetes, contributing to the therapeutic effects of the drug. Nat Med. 2017; 23: 850-858.

73. Pineiro $M$, Stanton $C$. Probiotic bacteria: Legislative framework-- requirements to evidence basis. J Nutr. 2007; 137: 850S-853S. 
74. Chapman CM, Gibson GR, Rowland I. Health benefits of probiotics: Are mixtures more effective than single strains? Eur J Nutr. 2011; 50: 1-17.

75. Pokrzywnicka P, Gumprecht J. Intestinal microbiota and its relationship with diabetes and obesity. Clin Diabetol. 2017; 5: 164-172.

76. Rijkers GT, Bengmark S, Enck P, Haller D, Herz U, Kalliomaki M, et al. Guidance for substantiating the evidence for beneficial effects of probiotics: Current status and recommendations for future research. J Nutr. 2010; 140: 671S-676S.

77. Cani PD, Neyrinck AM, Fava F, Knauf C, Burcelin RG, Tuohy KM, et al. Selective increases of bifidobacteria in gut microflora improve high-fat-diet-induced diabetes in mice through a mechanism associated with endotoxaemia. Diabetologia. 2007; 50: 2374-2383.

78. Boto-Ordonez M, Urpi-Sarda M, Queipo-Ortuno MI, Tulipani S, Tinahones FJ, Andres-Lacueva C. High levels of Bifidobacteria are associated with increased levels of anthocyanin microbial metabolites: A randomized clinical trial. Food Funct. 2014; 5: 1932-1938.

79. Yadav H, Jain S, Sinha PR. Antidiabetic effect of probiotic dahi containing Lactobacillus acidophilus and Lactobacillus casei in high fructose fed rats. Nutrition. 2007; 23: 62-68.

80. Yadav H, Lee JH, Lloyd J, Walter P, Rane SG. Beneficial metabolic effects of a probiotic via butyrate-induced GLP-1 hormone secretion. J Biol Chem. 2013; 288: 25088-25097.

81. Gibson GR, Roberfroid MB. Dietary modulation of the human colonic microbiota: Introducing the concept of prebiotics. J Nutr. 1995; 125: 1401-1412.

82. Meyer $D$, Stasse-Wolthuis $M$. The bifidogenic effect of inulin and oligofructose and its consequences for gut health. Eur J Clin Nutr. 2009; 63: 1277-1289.

83. Davis LM, Martinez I, Walter J, Goin C, Hutkins RW. Barcoded pyrosequencing reveals that consumption of galactooligosaccharides results in a highly specific bifidogenic response in humans. PloS one. 2011; 6: e25200.

84. Strowski MZ, Wiedenmann B. Probiotic carbohydrates reduce intestinal permeability and inflammation in metabolic diseases. Gut. 2009; 58: 1044-1045.

85. Cani PD, Bibiloni R, Knauf C, Waget A, Neyrinck AM, Delzenne NM, et al. Changes in gut microbiota control metabolic endotoxemia-induced inflammation in high-fat diet-induced obesity and diabetes in mice. Diabetes. 2008; 57: 1470-1481.

86. Cani PD, Lecourt E, Dewulf EM, Sohet FM, Pachikian BD, Naslain D, et al. Gut microbiota fermentation of prebiotics increases satietogenic and incretin gut peptide production with consequences for appetite sensation and glucose response after a meal. Am J Clin Nutr. 2009; 90: 1236-1243.

87. Queipo-Ortuno MI, Boto-Ordonez M, Murri M, Gomez-Zumaquero JM, Clemente-Postigo M, Estruch $\mathrm{R}$, et al. Influence of red wine polyphenols and ethanol on the gut microbiota ecology and biochemical biomarkers. Am J Clin Nutr. 2012; 95: 1323-1334.

88. Clemente-Postigo $M$, Queipo-Ortuno MI, Boto-Ordonez M, Coin-Araguez L, Roca-Rodriguez MM, Delgado-Lista J, et al. Effect of acute and chronic red wine consumption on lipopolysaccharide concentrations. Am J Clin Nutr. 2013; 97: 1053-1061.

89. Maury CP, Triana MH, Landín AA, Huergo CC, Pianesi M. Caracterización y evaluación nutricional de las dietas macrobióticas Ma-Pi. Revista Cubana De Investigaciones Biomédicas. 2008; 27-36

90. Khoruts A, Dicksved J, Jansson JK, Sadowsky MJ. Changes in the composition of the human fecal microbiome after bacteriotherapy for recurrent Clostridium difficile-associated diarrhea. J Clin Gastroenterol. 2010; 44: 354-360. 
91. Tvede M, Rask-Madsen J. Bacteriotherapy for chronic relapsing Clostridium difficile diarrhoea in six patients. Lancet. 1989; 1: 1156-1160.

92. Rohr MW, Narasimhulu CA, Rudeski-Rohr TA, Parthasarathy S. Negative Effects of a High-Fat Diet on Intestinal Permeability: A Review. Adv Nutr. 2019; 11: 77-91.

93. Ahola AJ, Lassenius MI, Forsblom C, Harjutsalo V, Lehto M, Groop PH. Dietary patterns reflecting healthy food choices are associated with lower serum LPS activity. Sci Rep. 2017; 7: 6511.

94. Saito T, Hayashida H, Furugen R. Metabolic endotoxemia initiates obesity and insulin resistance: Diabetes 56: 1761-1772. Diabetes. 2007; 56: e20.

95. Cani PD, Delzenne NM. The role of the gut microbiota in energy metabolism and metabolic disease. Curr Pharm Des. 2009; 15: 1546-1558.

96. Cani PD, Delzenne NM. Gut microflora as a target for energy and metabolic homeostasis. Curr Opin Clin Nutr Metab Care. 2007; 10: 729.

97. Bäckhed F, Normark S, Schweda EK, Oscarson S, Richter-Dahlfors A. Structural requirements for TLR4-mediated LPS signalling: A biological role for LPS modifications. Microbes Infect. 2003; 5: 1057-1063.

98. Bäckhed F. Gut Microbiota in Metabolic Syndrome. A Systems Biology Approach to Study Metabolic Syndrome. 2014; pp: 171-181.

99. Ghanim H, Abuaysheh S, Sia CL, Korzeniewski K, Chaudhuri A, Fernandez-Real JM, et al. Increase in plasma endotoxin concentrations and the expression of Toll-like receptors and suppressor of cytokine signaling-3 in mononuclear cells after a high-fat, high-carbohydrate meal: Implications for insulin resistance. Diabetes Care. 2009; 32: 2281-2287.

100. Anderson PD, Mehta NN, Wolfe ML, Hinkle CC, Pruscino L, Comiskey LL, et al. Innate immunity modulates adipokines in humans. J Clin Endocrinol Metab. 2007; 92: 2272-2279.

101.Cani PD, Osto M, Geurts L, Everard A. Involvement of gut microbiota in the development of lowgrade inflammation and type 2 diabetes associated with obesity. Gut Microbes. 2012; 3: 279288.

102. Turnbaugh PJ, Hamady M, Yatsunenko T, Cantarel BL, Duncan A, Ley RE, et al. A core gut microbiome in obese and lean twins. Nature. 2009; 457: 480-484.

103. De La Serre CB, Ellis CL, Lee J, Hartman AL, Rutledge JC, Raybould HE. Propensity to high-fat diet-induced obesity in rats is associated with changes in the gut microbiota and gut inflammation. Am J Physiol-Gastrointestinal Liver Physiol. 2010; 299: 440-448.

104.Geurts L, Neyrinck AM, Delzenne NM, Knauf C, Cani PD. Gut microbiota controls adipose tissue expansion, gut barrier and glucose metabolism: Novel insights into molecular targets and interventions using prebiotics. Benef Microbes. 2014; 5: 3-17.

105. Matias I, Di Marzo V. Endocannabinoids and the control of energy balance. Trends Endocrinol Metab. 2007; 18: 27-37.

106. Lau E, Carvalho D, Pina-Vaz C, Barbosa JA, Freitas P. Beyond gut microbiota: Understanding obesity and type 2 diabetes. Hormones. 2015; 14: 358-369.

107. Lambert DM, Muccioli GG. Endocannabinoids and related $\mathrm{N}$-acylethanolamines in the control of appetite and energy metabolism: Emergence of new molecular players. Curr Opin Clin Nutr Metab Care. 2007; 10: 735-744.

108. Muccioli GG. Endocannabinoid biosynthesis and inactivation, from simple to complex. Drug Discov Today Technol. 2010; 15: 474-483. 
109. Muccioli GG, Naslain D, Backhed F, Reigstad CS, Lambert DM, Delzenne NM, et al. The endocannabinoid system links gut microbiota to adipogenesis. Mol Syst Biol. 2010; 6: 392.

110.Alhamoruni A, Wright KL, Larvin M, O'Sullivan SE. Cannabinoids mediate opposing effects on inflammation-induced intestinal permeability. Br J Pharmacol. 2012; 165: 2598-2610.

111.Dray C, Knauf C, Daviaud D, Waget A, Boucher J, Buleon M, et al. Apelin stimulates glucose utilization in normal and obese insulin-resistant mice. Cell Metab. 2008; 8: 437-445.

112.Duparc T, Colom A, Cani PD, Massaly N, Rastrelli S, Drougard A, et al. Central apelin controls glucose homeostasis via a nitric oxide-dependent pathway in mice. Antioxid Redox Signal. 2011; 15: 1477-1496.

113.Geurts L, Lazarevic V, Derrien M, Everard A, Van Roye M, Knauf C, et al. Altered gut microbiota and endocannabinoid system tone in obese and diabetic leptin-resistant mice: Impact on apelin regulation in adipose tissue. Front Microbiol. 2011; 2: 149.

114. Koboziev I, Reinoso Webb C, Furr KL, Grisham MB. Role of the enteric microbiota in intestinal homeostasis and inflammation. Free Radic Biol Med. 2014; 68: 122-133.

115. Carding S, Verbeke K, Vipond DT, Corfe BM, Owen LJ. Dysbiosis of the gut microbiota in disease. Microb Ecol Health Dis. 2015; 26: 26191.

116. Anderson LA, Murphy SJ, Johnston BT, Watson RG, Ferguson HR, Bamford KB, et al. Relationship between Helicobacter pylori infection and gastric atrophy and the stages of the oesophageal inflammation, metaplasia, adenocarcinoma sequence: Results from the FINBAR case-control study. Gut. 2008; 57: 734-739.

117.Wang B, Yao M, Lv L, Ling Z, Li L. The human microbiota in health and disease. Engineering. 2017; 3: 71-82.

118.Schroeder BO, Backhed F. Signals from the gut microbiota to distant organs in physiology and disease. Nat Med. 2016; 22: 1079-1089.

119.Cani PD. Gut microbiota and obesity: Lessons from the microbiome. Brief Funct Genomics. 2013; 12: 381-387.

120.Wang L, Christophersen CT, Sorich MJ, Gerber JP, Angley MT, Conlon MA. Low relative abundances of the mucolytic bacterium Akkermansia muciniphila and Bifidobacterium spp. in feces of children with autism. Appl Environ Microbiol. 2011; 77: 6718-6721.

121.Tongju LI, Zhao Y, Dong G, Jia D, Baiping MA. The Gut Microbiota and Alzheimer's Disease. Isr J Health Policy Res. 2016; 58: 1.

122. Belkaid Y, Hand TW. Role of the microbiota in immunity and inflammation. Cell. 2014; 157: 121141.

123. Ohland CL, Macnaughton WK. Probiotic bacteria and intestinal epithelial barrier function. Am J Physiol Gastrointest Liver Physiol. 2010; 298: G807-819.

124.Gomes AC, Bueno AA, De Souza RG, Mota JF. Gut microbiota, probiotics and diabetes. Nutr J. 2014; 13: 60.

125.Suzuki T, Yamada T, Fujimura T, Kawamura F, Shimizu M, Yamashita R, et al. Diabetogenic effects of lymphocyte transfusion on the NOD or NOD nude mouse. J Jpn Soc. 1987; 16: 158161.

126.Giongo A, Gano KA, Crabb DB, Mukherjee N, Novelo LL, Casella G, et al. Toward defining the autoimmune microbiome for type 1 diabetes. ISME J. 2011; 5: 82. 
127. Murri M, Leiva I, Gomez-Zumaquero JM, Tinahones FJ, Cardona F, Soriguer F, et al. Gut microbiota in children with type 1 diabetes differs from that in healthy children: A case-control study. BMC Medicine. 2013; 11: 46.

128. De Goffau MC, Luopajaervi K, Knip M, Ilonen J, Ruohtula T, Haerkoenen T, et al. Fecal microbiota composition differs between children with $\beta$-Cell autoimmunity and those Without. Diabetes. 2013; 62: 1238-44.

129. Mejia-Leon ME, Petrosino JF, Ajami NJ, Dominguez-Bello MG, de la Barca AM. Fecal microbiota imbalance in Mexican children with type 1 diabetes. Sci Rep. 2014; 4: 3814.

130. Kosiewicz MM, Dryden GW, Chhabra A, Alard P. Relationship between gut microbiota and development of T cell associated disease. FEBS Lett. 2014; 588: 4195-4206.

131. Bibbò S, Dore MP, Pes GM, Delitala G, Delitala AP. Is there a role for gut microbiota in type 1 diabetes pathogenesis? Ann Med. 2016; 8: 11-22.

132. Brown CT, Davis-Richardson AG, Giongo A, Gano KA, Crabb DB, Mukherjee N, et al. Gut microbiome metagenomics analysis suggests a functional model for the development of autoimmunity for type 1 diabetes. PloS one. 2011; 6: e25792.

133. Hummel S, Veltman K, Cichon C, Sonnenborn U, Schmidt MA. Differential targeting of the ECadherin/beta-Catenin complex by gram-positive probiotic lactobacilli improves epithelial barrier function. Appl Environ Microbiol. 2012; 78: 1140-1147.

134.Singh R, Lei P, Andreadis ST. PKC- $\delta$ binds to E-cadherin and mediates EGF-induced cell scattering. Exp Cell Res. 2009; 315: 2899-2913.

135. Secondulfo M, lafusco D, Carratù R, Demagistris L, Sapone A, Generoso M, et al. Ultrastructural mucosal alterations and increased intestinal permeability in non-celiac, type I diabetic patients. Journal of the Italian Society of Gastroenterology \& the Italian Association for the Study of the Liver. 2004; 36: 35-45.

136. Bosi E, Molteni L, Radaelli MG, Folini L, Fermo I, Bazzigaluppi E, et al. Increased intestinal permeability precedes clinical onset of type 1 diabetes. Diabetologia. 2006; 49: 2824-2827.

137.Gülden E, Wong FS, Wen L. The gut microbiota and Type 1 Diabetes. Clin Immunol. 2015; 159: 143-153.

138. Vehik K, Dabelea D. The changing epidemiology of type 1 diabetes: Why is it going through the roof? Diabetes Metab Res Rev. 2011; 27: 3-13.

139. Round JL, Lee SM, Li J, Tran G, Jabri B, Chatila TA, et al. The Toll-like receptor 2 pathway establishes colonization by a commensal of the human microbiota. Science. 2011; 332: 974-977.

140. Mobegi FM, van Hijum SA, Burghout P, Bootsma HJ, de Vries SP, van der Gaast-de Jongh CE, et al. From microbial gene essentiality to novel antimicrobial drug targets. BMC Genomics. 2014; 15: 958.

141. Blandino G, Inturri R, Lazzara F, Di Rosa M, Malaguarnera L. Impact of gut microbiota on diabetes mellitus. Diabetes Metab. 2016; 42: 303-315.

142. Virtanen SM, Jaakko N, Carina KK, Suvi A, Heli T, Liisa U, et al. Food consumption and advanced $\beta$ cell autoimmunity in young children with HLA-conferred susceptibility to type 1 diabetes: $A$ nested case-control design. Am J Clin Nutr. 2012: 471-478.

143. Balzola F, Bernstein C, Ho GT, Lees C, Balzola F, Bernstein C, et al. Inducible Foxp3 + regulatory T-cell development by a commensal bacterium of the intestinal microbiota: Commentary. Proc Natl Acad Sci U S A . 2010; 107: 12204-12209. 
144. Martin-Orozco N, Chung Y, Chang SH, Wang YH, Dong C. Th17 cells promote pancreatic inflammation but only induce diabetes efficiently in lymphopenic hosts after conversion into Th1 cells. Eur J Immunol. 2010; 39: 216-224.

145.Ivanov, II, Littman DR. Modulation of immune homeostasis by commensal bacteria. Curr Opin Microbiol. 2011; 14: 106-114.

146. Aujla SJ, Dubin PJ, Kolls JK. Th17 cells and mucosal host defense. Semin Immunol. 2007; 19: 377382.

147.Atarashi K, Tanoue T, Oshima K, Suda W, Nagano Y, Nishikawa H, et al. Treg induction by a rationally selected mixture of Clostridia strains from the human microbiota. Nature. 2013; 500: 232-236.

148. Izcue A, Coombes JL, Powrie F. Regulatory lymphocytes and intestinal inflammation. Annu Rev Immunol. 2009; 27: 313-338.

149.Sartor RB. Microbial influences in inflammatory bowel diseases. Gastroenterology. 2008; 134: 577-594.

150. McInnes IB, Schett G. The pathogenesis of rheumatoid arthritis. N Engl J Med. 2011; 365: 22052219.

151. Vaahtovuo J, Munukka E, Korkeamaki M, Luukkainen R, Toivanen P. Fecal microbiota in early rheumatoid arthritis. J Rheumatol. 2008; 35: 1500-1505.

152. Coit $P$, Sawalha $A H$. The human microbiome in rheumatic autoimmune diseases: $A$ comprehensive review. Clin Immunol. 2016; 170: 70-79.

153.Shamriz O, Mizrahi H, Werbner M, Shoenfeld Y, Avni O, Koren O. Microbiota at the crossroads of autoimmunity. Autoimmun Rev. 2016; 15: 859-869.

154.Scher JU, Andrew S, Longman RS, Nicola S, Carles U, Craig B, et al. Expansion of intestinal Prevotella copri correlates with enhanced susceptibility to arthritis. Elife. 2013; 2: e01202 .

155.Liu X, Zou Q, Zeng B, Fang $\mathrm{Y}$, Wei $\mathrm{H}$. Analysis of fecal Lactobacillus community structure in patients with early rheumatoid arthritis. Curr Microbiol. 2013; 67: 170-176.

156.Scher JU, Sczesnak A, Longman RS, Segata N, Ubeda C, Bielski C, et al. Expansion of intestinal Prevotella copri correlates with enhanced susceptibility to arthritis. ELife. 2013; 2: e01202.

157. Chen J, Wright K, Davis JM, Jeraldo P, Marietta EV, Murray J, et al. An expansion of rare lineage intestinal microbes characterizes rheumatoid arthritis. Genome Medicine. 2016; 8: 43.

158. Chen $B$, Sun L, Zhang X. Integration of microbiome and epigenome to decipher the pathogenesis of autoimmune diseases. J Autoimmun. 2017; 83: 31-42.

159.Scher JU, Abramson SB. The microbiome and rheumatoid arthritis. Nat Rev Rheumatol. 2011; 7: 569-578.

160.Johnson S, Sidebottom D, Bruckner F, Collins D. Identification of Mycoplasma fermentans in synovial fluid samples from arthritis patients with inflammatory disease. J Clin Microbiol. 2000; 38: 90-93.

161. Newkirk MM, Zbar A, Baron M, Manges AR. Distinct bacterial colonization patterns of Escherichia coli subtypes associate with rheumatoid factor status in early inflammatory arthritis. Rheumatology. 2010; 49: 1311-1316.

162.Senior BW, Anderson GA, Morley KD, Kerr MA. Evidence that patients with rheumatoid arthritis have asymptomatic 'non-significant' Proteus mirabilis bacteriuria more frequently than healthy controls. J Infect. 1999; 38: 99-106. 
163.Sandberg ME, Bengtsson C, Klareskog L, Alfredsson L, Saevarsdottir S. Recent infections are associated with decreased risk of rheumatoid arthritis: A population-based case-control study. Ann Rheum Dis. 2015; 74: 904-907.

164. Ciccia F, Ferrante A, Guggino G, Triolo $G$. The role of the gastrointestinal tract in the pathogenesis of rheumatic diseases. Best Pract Res Clin Rheumatol. 2016; 30: 889-900.

165. Vaghef-Mehrabany E, Alipour B, Homayouni-Rad A, Sharif SK, Asghari-Jafarabadi M, Zavvari S. Probiotic supplementation improves inflammatory status in patients with rheumatoid arthritis. Nutrition. 2014; 30: 430-435.

166. Alipour B, Homayouni-Rad A, Vaghef-Mehrabany E, Sharif SK, Vaghef-Mehrabany L, AsghariJafarabadi $M$, et al. Effects of lactobacillus casei supplementation on disease activity and inflammatory cytokines in rheumatoid arthritis patients: A randomized double-blind clinical trial. Int J Rheum Dis. 2014; 17: 519-527.

167.Zamani B, Golkar HR, Farshbaf S, Emadi-Baygi M, Tajabadi-Ebrahimi M, Jafari P, et al. Clinical and metabolic response to probiotic supplementation in patients with rheumatoid arthritis: $A$ randomized, double-blind, placebo-controlled trial. Int J Rheum Dis. 2016; 19: 869-879.

168. He J, Li Y, Cao Y, Xue J, Zhou X. The oral microbiome diversity and its relation to human diseases. Folia Microbiologica. 2015; 60: 69-80.

169. Human Microbiome Project C. Structure, function and diversity of the healthy human microbiome. Nature. 2012; 486: 207-214.

170.Scher JU, Ubeda C, Equinda M, Khanin R, Buischi Y, Viale A, et al. Periodontal disease and the oral microbiota in new-onset rheumatoid arthritis. Arthritis Semin. 2012; 64: 3083-3094.

171.Guerreiro CS, Calado Â, Sousa J, Fonseca JE. Diet, Microbiota, and Gut Permeability - The Unknown Triad in Rheumatoid Arthritis. Front Med. 2018; 5: 349.

172.Zhang $X$, Zhang YD, Jia $H$, Feng $Q$, Wang $D$, Liang $D$, et al. The oral and gut microbiomes are perturbed in rheumatoid arthritis and partly normalized after treatment. Nat Med. 2015; 21: 895-905.

173. Colt $P$, Sawalha $A H$. The human microbiome in rheumatic autoimmune diseases: $A$ comprehensive review. Clin Immunol. 2016; 170: 70-79.

174. Björn W, Timo B, Cornelia F, Regina M, Norbert B, Hanns-Martin L, et al. Oral status in patients with early rheumatoid arthritis: A prospective, case-control study. Rheumatology. 2014: 526531.

175.Socransky SS, Haffajee AD, Cugini MA, Smith C, Kent RL. Microbial complexes in subgingival plaque. J Clin Periodontol. 2010; 25: 134-144.

176.Jump RL, Levine AD. Mechanisms of natural tolerance in the intestine. Inflamm Bowel Dis. 2004: 462-478.

177. Hold GL, Smith M, Grange C, Watt ER, El-Omar EM, Mukhopadhya I, et al. Role of the gut microbiota in inflammatory bowel disease pathogenesis: What have we learnt in the past 10 years? World J Gastroenterol. 2014; 20: 1192-1210.

178. Sekirov I, Russell SL, Antunes LC, Finlay BB. Gut Microbiota in health and disease. Physiol Rev. 2010; 90: 859-904.

179. Packey CD, Sartor RB. Interplay of commensal and pathogenic bacteria, genetic mutations, and immunoregulatory defects in the pathogenesis of inflammatory bowel diseases. J Intern Med. 2008; 263: 597-606. 
180.Chassaing B, Darfeuille-Michaud A. The commensal microbiota and enteropathogens in the pathogenesis of inflammatory bowel diseases. Gastroenterology. 2011; 140: 1720-1728. e1723.

181.Chassaing B, Darfeuille-Michaud A. The commensal microbiota and enteropathogens in the pathogenesis of inflammatory bowel diseases. Gastroenterology. 2011; 140: 1720-1728.

182. Wang W, Chen L, Zhou R, Wang X, Song L, Huang S, et al. Increased proportion of bifidobacterium and the lactobacillus group and loss of butyrate-producing bacteria in inflammatory bowel disease. J Clin Microbiol. 2013; 21: 21-24.

183.De la Fuent M, Franchi L, Araya D, Diaz-Jimenez D, Olivares M, Alvarez-Lobos M, et al. Escherichia coli isolates from inflammatory bowel diseases patients survive in macrophages and activate NLRP3 inflammasome. International. J Med Microbiol. 2015; 305: 925-936.

184. Frank DN, St Amand AL, Feldman RA, Boedeker EC, Harpaz N, Pace NR. Molecular-phylogenetic characterization of microbial community imbalances in human inflammatory bowel diseases.Proc Natl Acad Sci U S A. 2007; 104: 13780-13785.

185. Sokol H, Seksik P, Furet JP, Firmesse O, Nion-Larmurier I, Beaugerie L, et al. Low counts of Faecalibacterium prausnitzii in colitis microbiota. Inflamm Bowel Dis. 2009; 15: 1183-1189.

186. Manichanh C, Borruel N, Casellas F, Guarner F. The gut microbiota in IBD. Nat Rev Dis Primers. 2012; 9: 599-608.

187. Mukhopadhya I, Hansen R, El-Omar EM, Hold GL. IBD-what role do Proteobacteria play? Nat Rev Dis Primers. 2012; 9: 219-230.

188. Chassaing B, Rolhion N, De Vallée A, Sa'ad AY, Prorok-Hamon M, Neut C, et al. Crohn disease-associated adherent-invasive E. coli bacteria target mouse and human Peyer's patches via long polar fimbriae. J Clin Invest. 2011; 121: 966-975.

189. Ventham NT, Kennedy NA, Nimmo ER, Satsangi J. Beyond gene discovery in inflammatory bowel disease: The emerging role of epigenetics. Gastroenterology. 2013; 145: 293-308.

190.Png CW, Linden SK, Gilshenan KS, Zoetendal EG, McSweeney CS, Sly LI, et al. Mucolytic bacteria with increased prevalence in IBD mucosa augment in vitro utilization of mucin by other bacteria. Am J Gastroenterol. 2010; 105: 2420-2428.

191.Gevers D, Kugathasan S, Denson L, Vázquez-Baeza Y, Treuren WV, Ren B, et al. The treatmentnaive microbiome in new-onset Crohn's disease. Cell Host Microbe. 2014; 15: 382-392.

192. Hansen R, Thomson JM, El-Omar EM, Hold GL. The role of infection in the aetiology of inflammatory bowel disease. J Gastroenterol. 2010; 45: 266-276.

193. Mahendran V, Riordan SM, Grimm MC, Tran TA, Major J, Kaakoush NO, et al. Prevalence of Campylobacter species in adult Crohn's disease and the preferential colonization sites of Campylobacter species in the human intestine. PloS one. 2011; 6: e25417.

194. Mukhopadhya I, Thomson JM, Hansen R, Berry SH, El-Omar EM, Hold GL. Detection of Campylobacter concisus and other Campylobacter species in colonic biopsies from adults with ulcerative colitis. PloS one. 2011; 6: e21490.

195. Ohkusa T, Okayasu I, Ogihara T, Morita K, Ogawa M, Sato N. Induction of experimental ulcerative colitis by Fusobacterium varium isolated from colonic mucosa of patients with ulcerative colitis. Gut. 2003; 52: 79-83.

196. Lee IA, Kim DH. Klebsiella pneumoniae increases the risk of inflammation and colitis in a murine model of intestinal bowel disease. Scandinavian J Gastroenterol. 2011; 46: 684-693. 
197.Gradel KO, Nielsen HL, Schonheyder HC, Ejlertsen T, Kristensen B, Nielsen H. Increased shortand long-term risk of inflammatory bowel disease after salmonella or campylobacter gastroenteritis. Gastroenterology. 2009; 137: 495-501.

198.Leu SB, Shulman SC, Steelman CK, Lamps LW, Bulut OP, Abramowsky CR, et al. Pathogenic Yersinia DNA in intestinal specimens of pediatric patients with Crohn's disease. Fetal Pediatr Pathol. 2013; 32: 367-370.

199.Tursi A, Brandimarte G, Papa A, Giglio A, Elisei W, Giorgetti GM, et al. Treatment of relapsing mild-to-moderate ulcerative colitis with the probiotic VSL\#3 as adjunctive to a standard pharmaceutical treatment: A double-blind, randomized, placebo-controlled study. Am J Gastroenterol. 2010; 105: 2218-2227.

200.Feng T, Wang L, Schoeb TR, Elson CO, Cong Y. Microbiota innate stimulation is a prerequisite for T cell spontaneous proliferation and induction of experimental colitis. J Exp Med. 2010; 207: 1321-1332.

201.Ward JM, Anver MR, Haines DC, Melhorn JM, Fox JG. Inflammatory large bowel disease in immunodeficient mice naturally infected with Helicobacter hepaticus. J Exp Med. 1996; 46: 1520.

202. Onderdonk AB, Steeves RM, Cisneros RL, Bronson RT. Adoptive transfer of immune enhancement of experimental ulcerative colitis. Infect Immun. 1984; 46: 64-67.

203. Khor B, Gardet A, Xavier RJ. Genetics and pathogenesis of inflammatory bowel disease. Nature. 2011; 474: 307-317.

204. Hevia A, Milani C, Lopez P, Cuervo A, Arboleya S, Duranti S, et al. Intestinal dysbiosis associated with systemic lupus erythematosus. mBio. 2014; 5: e01548- e01514.

205.Luo XM, Edwards MR, Mu Q, Yu Y, Vieson MD, Rilly CM, et al. Gut microbiota in human systemic lupus erythematosus and a mouse model of lupus. Appl Environ Microbiol. 2018; 84: e0228817.

206. Rojo D, Hevia A, Bargiela R, López P, Cuervo A, González S, et al. Ranking the impact of human health disorders on gut metabolism: Systemic lupus erythematosus and obesity as study cases. Sci Rep. 2015; 5: 8310.

207. Mizanur M, Rahman S, Sudhir Z, Sing R, Zahra T, Golabkesh T, et al. IgM antibodies against malondialdehyde and phosphorylcholine are together strong protection markers for atherosclerosis in systemic lupus erythematosus: Regulation and underlying mechanisms. Clin Immunol. 2016; 166-167: 27-37.

208.Yacoub R, Jacob A, Wlaschin J, McGergor M, Quigg RJ, Alexander JJ. Lupus: The microbiome angle. Immunobiology. 2018; 223: 460-465.

209.Saadat YR, Hejazian M, Bastami M, Khatibi SM, Ardalan M, Vahed SZ. The role of microbiota in the pathogenesis of lupus: Dose it impact lupus nephritis? Pharmacol Res. 2019; 139: 191-198.

210. Mu Q, Zhang H, Liao X, Lin K, Liu H, Edwards MR, et al. Control of lupus nephritis by the gut microbiota. Microbiome. 2017; 5: 73-78.

211.Zhang H, Liao X, Sparks JB, Luo XM. Dynamics of gut microbiota in autoimmune lupus. Appl Environ Microbiol. 2014; 80: 7551-7560.

212.Tucker CF, Nebane-Ambe DL, Chhabra A, Parnell SA, Zhao Y, Alard P, et al. Decreased frequencies of $C D 4+C D 25+$ Foxp3+ cells and the potent $C D 103+$ subset in peripheral lymph nodes correlate with autoimmune disease predisposition in some strains of mice. Autoimmunity. 2011; 44: 453-464. 
213. Van Praet JT, Donovan E, Vanassche I, Drennan MB, Windels F, Dendooven A, et al. Commensal microbiota influence systemic autoimmune responses. EMBO J. 2015; 34: 466-474.

214.Johnson BM, Gaudreau MC, Al-Gadban MM, Gudi R, Vasu C. Impact of dietary deviation on disease progression and gut microbiome composition in lupus-prone SNF1 mice. Clin Exp Immunol. 2015; 181: 323-337.

215.Franco JS, Amaya-Amaya J, Anaya JM. Thyroid disease and autoimmune disease. Autoimmunity: from Bench to Bedside. Bogata: El Rosario University Press; 2013; Chapter 30.

216.Köhling HL, Plummer SF, Marchesi JR, Davidge KS, Ludgate M. The microbiota and autoimmunity: Their role in thyroid autoimmune diseases. Clin Immunol. 2017; 183: 63-74.

217.Penhale WJ, Young PR. The influence of the normal microbial flora on the susceptibility of rats to experimental autoimmune thyroiditis. Clin Exp Immunol. 1988; 72: 288-292.

218. Kiseleva E, Mikhailopulo K, Sviridov O, Novik G, Knirel Y, Szwajcer Dey E. The role of components of Bifidobacterium and Lactobacillus in pathogenesis and serologic diagnosis of autoimmune thyroid diseases. Benef Microbes. 2011.

219.Zhou JS, Gill HS. Immunostimulatory probiotic Lactobacillus rhamnosus HNO01 and Bifidobacterium lactis HN019 do not induce pathological inflammation in mouse model of experimental autoimmune thyroiditis. Int J Food Microbiol. 2005; 103: 97-104.

220. Fors G. Understanding hyperthyroidism: The gut link. DC Pract Insights. 2013.

221.Zhao F, Fuya J, Feng J, Jing L, Li Y, Jun H, et al. Alterations of the gut microbiota in Hashimoto's thyroiditis patients. Thyroid. 2018; 28: 175-186

222. Ishaq HM, Mohammad IS, Guo H, Shahzad M, Hou YJ, Ma C, et al. Molecular estimation of alteration in intestinal microbial composition in Hashimoto's thyroiditis patients. Biomed Pharmacother. 2017; 95: 865-874.

223. Cristiano LE, Lisa BA, Maurizio G, Emidio S, Andrea L, Antonio L, et al. Association between hypothyroidism and small intestinal bacterial overgrowth. J Clin Endocrinol Metab. 2007; 92: 4180-4184.

224. Matsuoka K, Kanai T. The gut microbiota and inflammatory bowel disease. Seminars in Immunopathology. Semin Immunopathol. 2015; 37: 47-55.

225. Kanai T, Mikami Y, Hayashi A. A breakthrough in probiotics: Clostridium butyricum regulates gut homeostasis and anti-inflammatory response in inflammatory bowel disease. J Gastroenterol. 2015; 50: 928-939.

226. Mori K, Nakagawa Y, Ozaki H. Does the gut microbiota trigger Hashimoto's thyroiditis? Discov Med. 2012; 14: 321-326.

227.Virili C, Fallahi P, Antonelli A, Benvenga S, Centanni M. Gut microbiota and Hashimoto's thyroiditis. Rev Endocr Metab Disord. 2018; 19: 293-300.

228. Virili C, Centanni M. Does microbiota composition affect thyroid homeostasis? Endocrine. 2015; 49: 583-587.

229. Ishaq HM, Mohammad IS, Shahzad M, Ma C, Raza MA, Wu X, et al. Molecular alteration analysis of human gut microbial composition in graves' disease patients. Int J Biol Sci. 2018; 14: 15581570.

230. Kristensen B. Regulatory B and T cell responses in patients with autoimmune thyroid disease and healthy controls. Dan Med J. 2016; 63-65.

231.Bjorksten B, Naaber P, Sepp E, Mikelsaar M. The intestinal microflora in allergic Estonian and Swedish 2-year-old children. Clin Exp Allergy. 1999; 29: 342-346. 
232. Kirjavainen PV, Apostolou E, Arvola T, Salminen SJ, Gibson GR, Isolauri E. Characterizing the composition of intestinal microflora as a prospective treatment target in infant allergic disease. FEMS Immunol Med Microbiol. 2001; 32: 1-7.

233.Bjorkstén B, Sepp E, Julge K, Voor T, Mikelsaar M. Allergy development and the intestinal microflora during the first year of life. J Allergy Clin Immunol. 2001; 108: 516-520.

234.Johansson MA, Sjogren YM, Presson JO, Nilsson C, Sverremark-Ekstrom E. Early colonization with a group of lactobacilli decreases the risk for allergy at five years of age despite allergic heredity. PloS one. 2011; 6: e23031.

235. Verhulst SL, Vael C, Beunckens C, Nelen V, Goossens H, Desager K. A Longitudinal analysis on the association between antibiotic use, intestinal microflora, and wheezing during the first year of life. J Asthma Res. 2008; 45: 828-832.

236. Van Nimwegen FA, Penders J, Stobberingh EE, Postma DS, Koppelman GH, Kerkhof M, et al. Mode and place of delivery, gastrointestinal microbiota, and their influence on asthma and atopy. J Allergy Clin Immunol 2011; 128: 948-955.

237.Zhao L. The gut microbiota and obesity: From correlation to causality. Nat Rev Microbiol. 2013; 11: 639.

238.Turnbaugh PJ, BaCkhed F, Fulton L, Gordon JI. Diet-induced obesity is linked to marked but reversible alterations in the mouse distal gut microbiome. Cell Host Microbe. 2008; 31: 213223.

239.Żak-Gołąb A, OLszanecka-Glinianowicz M, Kocełak P, Chudek J. Rola flory jelitowej w patogenezie otyłości. Postepy Hig Med Dosw (Online). 2014; 68: 84-95.

240.Jumpertz R, Le DS, Turnbaugh PJ, Trinidad C, Bogardus C, Gordon Jl, et al. Energy-balance studies reveal associations between gut microbes, caloric load, and nutrient absorption in humans. Am J Clin Nutr. 2011; 94: 58-65.

241.Collado MC, Isolauri E, Laitinen K, Salminen S. Distinct composition of gut microbiota during pregnancy in overweight and normal-weight women. Am J Clin Nutr. 2008; 88: 894-899.

242. KalliomaKi M, Carmen Collado M, Salminen S, Isolauri E. Early differences in fecal microbiota composition in children may predict overweight. Am J Clin Nutr. 2008; 87: 534-538.

243. Balamurugan R, George G, Kabeerdoss J, Hepsiba J, Chandragunasekaran AM, Ramakrishna BS. Quantitative differences in intestinal Faecalibacterium prausnitzii in obese Indian children. $\mathrm{Br} \mathrm{J}$ Nutr. 2010; 103: 335-338.

244.Santacruz A, Collado MC, García-Valdés L, Segura MT, Sanz Y. Gut microbiota composition is associated with body weight, weight gain and biochemical parameters in pregnant women. $\mathrm{Br}$ J Nutr. 2010; 104: 83-92.

245. Nadal I, Santacruz A, Marcos A, Warnberg J, Sanz Y. Shifts in clostridia, bacteroides and immunoglobulin-coating fecal bacteria associated with weight loss in obese adolescents. Int J Obes. 2009; 33: 758-767.

246. Kristina H, Amira K, Geneviève M, Chou CJ. Is the Gut Microbiota a new factor contributing to obesity and its metabolic disorders? J Obes. 2012; 201: 879151.

247.Schwiertz A, Taras D, Schafer K, Beijer S, Bos NA, Donus C, et al. Microbiota and SCFA in lean and overweight healthy subjects. Obesity (Silver Spring). 2010; 18: 190-195.

248. Petra Al, Panagiotidou S, Hatziagelaki E, Stewart JM, Conti P, Theoharides TC. Gut-microbiotabrain axis and its effect on neuropsychiatric disorders with suspected immune dysregulation. Clin Ther. 2015; 37: 984-995. 
249. Graessler J, Qin Y, Zhong H, Zhang J, Licinio J, Wong ML, et al. Metagenomic sequencing of the human gut microbiome before and after bariatric surgery in obese patients with type 2 diabetes: Correlation with inflammatory and metabolic parameters. Pharmacogenomics J. 2013; 13: $514-$ 522.

250.Everard A, Belzer C, Geurts L, Ouwerkerk JP, Cani PD. Cross-talk between Akkermansia muciniphila and intestinal epithelium controls diet-induced obesity. Proc Natl Acad Sci U S A. 2013; 110: 9066-9071.

251. Everard A, Lazarevic V, Derrien M, Girard M, Muccioli GG, Neyrinck AM, et al. Responses of gut microbiota and glucose and lipid metabolism to prebiotics in genetic obese and diet-Induced leptin-resistant mice. Diabetes. 2011; 60: 2775-2786.

252. Backhed F, Ding H, Wang T, Hooper LV, Koh GY, Nagy A, et al. The gut microbiota as an environmental factor that regulates fat storage. Proc Natl Acad Sci U S A. 2004; 101: 1571815723.

253. Backhed F, Manchester JK, Semenkovich CF, Gordon JI. Mechanisms underlying the resistance to diet-induced obesity in germ-free mice.Proc Natl Acad Sci U S A. 2007; 104: 979-984.

254.Cani PD, Joly E, Horsmans Y, Delzenne NM. Oligofructose promotes satiety in healthy human: $A$ pilot study. Eur J Clin Nutr. 2006; 60: 567-572.

255.Verhoef SP, Meyer D, Westerterp KR. Effects of oligofructose on appetite profile, glucagon-like peptide 1 and peptide YY3-36 concentrations and energy intake. Br J Nutr. 2011; 106: 17571762.

256. Qin J, Li Y, Cai Z, Li S, Zhu J, Zhang F, et al. A metagenome-wide association study of gut microbiota in type 2 diabetes. Nature. 2012; 490: 55-60.

257. Karlsson FH, Tremaroli V, Nookaew I, Bergstroem G, Behre CJ, Fagerberg B, et al. Gut metagenome in European women with normal, impaired and diabetic glucose control. Nature. 2013; 498: 99-103.

258. Brahe LK, Le Chatelier E, Prifti E, Pons N, Kennedy S, Hansen T, et al. Specific gut microbiota features and metabolic markers in postmenopausal women with obesity. Nutr Diabe. 2015; 5: e159.

259. Pedersen HK, Gudmundsdottir V, Nielsen HB, Hyotylainen T, Nielsen T, Jensen BA, et al. Human gut microbes impact host serum metabolome and insulin sensitivity. Nature. 2016; 535: 376381.

260. Festi D, Schiumerini R, Eusebi LH, Marasco G, Taddia M, Colecchia A. Gut microbiota and metabolic syndrome. World J Gastroenterol. 2014; 20: 16079-16094.

261. Creely SJ, McTernan PG, Kusminski CM, Fisher FM, Da Silva NF, Khanolkar M, et al. Lipopolysaccharide activates an innate immune system response in human adipose tissue in obesity and type 2 diabetes. Am J Physiol Endocrinol Metab. 2007; 292: E740-747.

262.Spruss A, Kanuri G, Wagnerberger S, Haub S, Bischoff SC, Bergheim I. Toll-like receptor 4 is involved in the development of fructose-induced hepatic steatosis in mice. Hepatology. 2009; 50: 1094-1104.

263.Cani PD, Possemiers S, Van de Wiele T, Guiot Y, Everard A, Rottier O, et al. Changes in gut microbiota control inflammation in obese mice through a mechanism involving GLP-2-driven improvement of gut permeability. Gut. 2009; 58: 1091-1103.

264. Hughes HK, Rose D, Ashwood P. The gut microbiota and dysbiosis in autism spectrum disorders. Curr Neurol Neurosci Rep. 2018; 18: 1-15. 
265. Fattorusso A, Di Genova L, Dell'Isola GB, Mencaroni E, Esposito S. Autism spectrum disorders and the gut microbiota. Nutrients. 2019; 11: 521-525.

266. Srikantha P, Mohajeri MH. The Possible Role of the Microbiota-Gut-Brain-Axis in autism spectrum disorder. Int J Mol Sci. 2019; 20: 2115-2120.

267.Pulikkan J, Mazumder A, Grace T. Role of the Gut microbiome in autism spectrum disorders. Adv Exp Med Bio. 2019; 1118: 253-269.

268. Bolte ER. Autism and Clostridium tetani. Med Hypotheses. 1998; 51: 133-144.

269. Parracho HM, Bingham MO, Gibson GR, McCartney AL. Differences between the gut microflora of children with autistic spectrum disorders and that of healthy children. J Med Microbiol. 2005; 54: 987-991.

270. Kesli R, Gokcen C, Bulug U, Terzi Y. Investigation of the relation between anaerobic bacteria genus clostridium and late-onset autism etiology in children. J Immunoassay Immunochem. 2014; 35: 101-109.

271. Finegold SM, Molitoris D, Song Y, Liu C, Vaisanen ML, Bolte E, et al. Gastrointestinal microflora studies in late-onset autism. Clinical infectious diseases : An official publication of the Infectious Diseases Society of America. Clin Infect Dis. 2002; 35: S6-S16.

272.Song Y, Liu C, Finegold SM. Real-time PCR quantitation of clostridia in feces of autistic children. Appl Environ Microbiol. 2004; 70: 6459-6465.

273.Shultz SR, MacFabe DF, Ossenkopp KP, Scratch S, Whelan J, Taylor R, et al. Intracerebroventricular injection of propionic acid, an enteric bacterial metabolic end-product, impairs social behavior in the rat: Implications for an animal model of autism. Neuropharmacology. 2008; 54: 901-911.

274.Pulikkan J, Maji A, Dhakan DB, Saxena R, Mohan B, Anto MM, et al. Gut microbial dysbiosis in Indian children with autism spectrum disorders. Microb Ecol. 2018; 76: 1102-1114.

275.De Angelis M, Francavilla R, Piccolo M, De Giacomo A, Gobbetti M. Autism spectrum disorders and intestinal microbiota. Gut Microbes. 2015; 6: 207-213.

276. Finegold SM, Dowd SE, Gontcharova V, Liu C, Henley KE, Wolcott RD, et al. Pyrosequencing study of fecal microflora of autistic and control children. Anaerobe. 2010; 16: 444-453.

277.Tomova A, Husarova V, Lakatosova S, Bakos J, Vlkova B, Babinska K, et al. Gastrointestinal microbiota in children with autism in Slovakia. Physiol Behav. 2015; 138: 179-187.

278. Wang L, Christophersen CT, Sorich MJ, Gerber JP, Angley MT, Conlon MA. Increased abundance of Sutterella spp. and Ruminococcus torques in feces of children with autism spectrum disorder. Mol Autism. 2013; 4: 42-50.

279. Finegold SM, Downes J, Summanen PH. Microbiology of regressive autism. Anaerobe. 2012; 18 : 260-262.

280. De Angelis M, Piccolo M, Vannini L, Siragusa S, De Giacomo A, Serrazzanetti DI, et al. Fecal microbiota and metabolome of children with autism and pervasive developmental disorder not otherwise specified. PloS one. 2013; 8: e76993.

281.Liu F, Li J, Wu F, Zheng H, Peng Q, Zhou H. Altered composition and function of intestinal microbiota in autism spectrum disorders: A systematic review. Transl Psychiatry. 2019; 9: 43.

282. Williams BL, Hornig M, Parekh T, Lipkin WI. Application of novel PCR-based methods for detection, quantitation, and phylogenetic characterization of Sutterella species in intestinal biopsy samples from children with autism and gastrointestinal disturbances. mBio. 2012; 3. 
283. Kushak RI, Winter HS, Buie TM, Cox SB, Phillips CD, Ward NL. Analysis of the Duodenal Microbiome in autistic individuals: Association with carbohydrate digestion. J Pediatr Gastroenterol Nutr. 2017; 64: e110-e116.

284. lovene MR, Bombace F, Maresca R, Sapone A, lardino P, Picardi A, et al. Intestinal dysbiosis and yeast isolation in stool ofsubjects with autism spectrum disorders. Mycopathologia. 2017; 182: 349-363.

285.Strati F, Cavalieri D, Albanese D, De Felice C, Donati C, Hayek J, et al. New evidences on the altered gut microbiota in autism spectrum disorders. Microbiome. 2017; 5: 24-24.

286. Choi GB, Yim YS, Wong H, Kim S, Kim H, Kim SV, et al. The maternal interleukin-17a pathway in mice promotes autism-like phenotypes in offspring. Science. 2016; 351: 933-939.

287. Yang Y, Tian J, Yang B. Targeting gut microbiome: A novel and potential therapy for autism. Life sci. 2018; 194: 111-119.

288. Kang DW, Adams JB, Coleman DM, Pollard EL, Maldonado J, McDonough-Means S, et al. Longterm benefit of Microbiota Transfer Therapy on autism symptoms and gut microbiota. Sci Rep. 2019; 9: 5821-5827.

289.Bull-Larsen S, Mohajeri $\mathrm{MH}$. The potential influence of the bacterial microbiome on the development and progression of ADHD. Nutrients. 2019; 11: 2805-2811.

290.Wang LJ, Yang CY, Chou WJ, Lee MJ, Chou MC, Kuo HC, et al. Gut microbiota and dietary patterns in children with attention-deficit/hyperactivity disorder. Eur Child Adolesc Psychiatry. 2019; 29: 287-297.

291. Howard AL, Robinson M, Smith GJ, Ambrosini GL, Piek JP, Oddy WH. ADHD is associated with a "Western" dietary pattern in adolescents. J Atten Disord. 2011; 15: 403-411.

292. Millichap JG, Yee MM. The diet factor in attention-deficit/hyperactivity disorder. Pediatrics. 2012; 129: 330-337.

293. Aarts E, Ederveen TH, Naaijen J, Zwiers MP, Boekhorst J, Timmerman HM, et al. Gut microbiome in ADHD and its relation to neural reward anticipation. PloS one. 2017; 12: e0183509.

294. Dougherty DD, Bonab AA, Spencer TJ, Rauch SL, Madras BK, Fischman AJ. Dopamine transporter density in patients with attention deficit hyperactivity disorder. Lancet. 1999; 354: 2132-2133.

295.Jiang HY, Zhou YY, Zhou GL, Li YC, Yuan J, Li XH, et al. Gut microbiota profiles in treatment-naive children with attention deficit hyperactivity disorder. Behav Brain Res. 2018; 347: 408-413.

296. Christian LM, Galley JD, Hade EM, Schoppe-Sullivan S, Kamp Dush C, Bailey MT. Gut microbiome composition is associated with temperament during early childhood. Brain Behav Immun. 2015; 45: 118-127.

297. Buske-Kirschbaum A, Schmitt J, Plessow F, Romanos M, Weidinger S, Roessner V. Psychoendocrine and psychoneuroimmunological mechanisms in the comorbidity of atopic eczema and attention deficit/hyperactivity disorder. Psychoneuroendocrinology. 2013; 28: 1223.

298. Alexander PK, Alexandra Z, Lukas T, Wolfgang L, Stefan S, Lioba B, et al. Reduced microbiome alpha diversity in young patients with ADHD. PloS one. 2018; 13: e0200728.

299. PäRtty A, KalliomäKi M, Wacklin P, Salminen S, Isolauri E. A possible link between early probiotic intervention and the risk of neuropsychiatric disorders later in childhood: A randomized trial. Pediatr Res. 2015; 77: 823-828. 
300. Severance EG, Gressitt KL, Stallings CR, Origoni AE, Khushalani S, Leweke FM, et al. Discordant patterns of bacterial translocation markers and implications for innate immune imbalances in schizophrenia. Schizophr Res. 2013; 148: 130-137.

301.Kirkpatrick B, Miller BJ. Inflammation and schizophrenia. Schizophrenia Bulletin. 2013; 39: 1174-1179.

302. Shen Y, Xu J, Li Z, Huang Y, Liang Y. Analysis of gut microbiota diversity and auxiliary diagnosis as a biomarker in patients with schizophrenia: A cross-sectional study. Schizophr Res. 2018; 197: 470-477.

303. Nguyen TT, Kosciolek T, Maldonado Y, Daly RE, Martin AS, McDonald D, et al. Differences in gut microbiome composition between persons with chronic schizophrenia and healthy comparison subjects. Schizophr Res. 2018; 204: 23-29.

304.Zheng P, Zeng B, Liu M, Chen J, Pan J, Han Y, et al. The gut microbiome from patients with schizophrenia modulates the glutamate-glutamine-GABA cycle and schizophrenia-relevant behaviors in mice. Sci Adv. 2019; 5: eaau8317.

305. Mortensen PB, NoRgaard-Pedersen B, Waltoft BL, SoRensen TL, Hougaard D, Torrey EF, et al. Toxoplasma gondii as a risk factor for early-onset schizophrenia: Analysis of filter paper blood samples obtained at birth. Biol Psychiatry. 2007; 61: 688-693.

306. Castronallar E, Bendall ML, Pérezlosada M, Sabuncyan S, Severance EG, Dickerson FB, et al. Composition, taxonomy and functional diversity of the oropharynx microbiome in individuals with schizophrenia and controls. Peer J. 2015; 3: e1140.

307. Bastiaanssen TF, Cowan CS, Claesson MJ, Dinan TG, Cryan JF. Making Sense of ... the Microbiome in Psychiatry. Int J Neuropsychopharmacol. 2019; 22: 37-52.

308.Sánchez-Villegas A, Martínez-González MÁ, Estruch R, Salas Salvadó J, Corella D, Covas MI, et al. Mediterranean dietary pattern and depression: The PREDIMED randomized trial. BMC Med. 2013; 11: 208-208.

309.Dash S, Clarke G, Berk M, Jacka FN. The gut microbiome and diet in psychiatry: Focus on depression. Curr Opin Psychiatry. 2015; 28: 1-6.

310.Jiang $H$, Ling $Z$, Zhang $Y$, Mao $H$, Ma Z, Yin $Y$, et al. Altered fecal microbiota composition in patients with major depressive disorder. Brain Behav Immun. 2015; 48: 186-194.

311.Butler MI, MörkI S, Sandhu KV, Cyran JF, Dinan TG. The gut microbiome and mental health: What should we tell our patients? Can J Psychiatry.2019; 64: 746-760.

312.Zheng $P$, Zeng $B$, Zhou $C$, Liu M, Fang $Z, X u X$, et al. Gut microbiome remodeling induces depressive-like behaviors through a pathway mediated by the host's metabolism. Mol Psychiatry. 2016; 21: 786-796.

313. Aizawa E, Tsuji H, Asahara T, Takahashi T, Teraishi T, Yoshida S, et al. Possible association of Bifidobacterium and Lactobacillus in the gut microbiota of patients with major depressive disorder. J Affect Disord. 2016; 202: 254-257.

314. Naseribafrouei A, Hestad K, Avershina E, Sekelja M, LinloKken A, Wilson R, et al. Correlation between the human fecal microbiota and depression. Neurogastroenterol Motil. 2014; 26: 1155-1162.

315. Vetulani J. Early maternal separation: A rodent model of depression and a prevailing human condition. Pharmacol Rep. 2013; 65: 1451-1461.

316. Phillips JGP. The Treatment of Melancholia by the Lactic Acid Bacillus. Br J Psychiatr. 1910; 56: 422-431. 
317. Huang $\mathrm{R}$, Wang $\mathrm{K}$, Hu J. A systematic review and meta-analysis of randomized controlled trials. Nutrients. 2016; 10: 483-495.

318. Fond G, Boukouaci W, Chevalier G, Regnault A, Eberl G, Hamdani N, et al. The "psychomicrobiotic": Targeting microbiota in major psychiatric disorders: A systematic review. Pathol Biol. 2015; 63: 35-42.

319. Kim N, Yun M, Oh YJ, Choi HJ. Mind-altering with the gut: Modulation of the gut-brain axis with probiotics. J Microbiol. 2018; 56: 172-182.

320.Liu RT, Walsh RF, Sheehan AE. Prebiotics and probiotics for depression and anxiety: A systematic review and meta-analysis of controlled clinical trials. Neurosci Biobehav Rev. 2019; 102: 13-23.

321. Evans SJ, Bassis CM, Hein R, Assari S, Flowers SA, Kelly MB, et al. The gut microbiome composition associates with bipolar disorder and illness severity. Journal of Psychiatric Research. 2017; 87: 23-29.

322.Painold A, Morkl S, Kashofer K, Halwachs B, Dalkner N, Bengesser S, et al. A step ahead: Exploring the gut microbiota in patients with bipolar disorder during a depressive episode. Bipolar Disord. 2019; 21: 40-49.

323. Flowers SA, Evans SJ, Ward KM, Mcinnis MG, Ellingrod VL. Interaction between atypical antipsychotics and the gut microbiome in a bipolar disease cohort. Pharmacotherapy. 2016; 37: 261-267.

324.Coello K, Hansen TH, Sorensen N, Munkholm K, Kessing LV, Pedersen O, et al. Gut microbiota composition in patients with newly diagnosed bipolar disorder and their unaffected first-degree relatives. Brain Behav Immun. 2018; 75: 112-118.

325. Reininghaus EZ, Wetzlmair LC, Fellendorf FT, Platzer M, Queissner R, Birner A, et al. The impact of probiotic supplements on cognitive parameters in euthymic individuals with bipolar disorder: A pilot study. Neuropsychobiology. 2018: 1-8.

326. Dickerson F, Adamos M, Katsafanas E, Khushalani S, Origoni A, Savage C, et al. Adjunctive probiotic microorganisms to prevent rehospitalization in patients with acute mania: $A$ randomized controlled trial. Bipolar Disord. 2018; 20: 614-621.

327. Yang B, Wei J, Ju P, Chen J. Effects of regulating intestinal microbiota on anxiety symptoms: $A$ systematic review. General Psychiatry. 2019; 32: e100056.

328. Foster JA, Neufeld KA. Gut-brain axis: How the microbiome influences anxiety and depression. Trends Neurosci. 2013; 36: 305-312.

329. Stevens BR, Goel R, Seungbum K, Richards EM, Holbert RC, Pepine CJ, et al. Increased human intestinal barrier permeability plasma biomarkers zonulin and FABP2 correlated with plasma LPS and altered gut microbiome in anxiety or depression. Gut. 2018; 67: 1555-1557.

330. Kleiman SC, Bulik-Sullivan EC, Glenny EM, Zerwas SC, Huh EY, Tsilimigras MC, et al. The Gutbrain axis in healthy females: Lack of significant association between microbial composition and diversity with psychiatric measures. PloS one. 2017; 12: e0170208.

331.Jiang HY, Zhang X, Yu ZH, Zhang Z, Deng M, Zhao JH, et al. Altered gut microbiota profile in patients with generalized anxiety disorder. J Psychiatr Res. 2018; 104: 130-136.

332. Pfleiderer A, Lagier JC, Armougom F, Robert C, Vialettes B, Raoult D. Culturomics identified 11 new bacterial species from a single anorexia nervosa stool sample. Eur J Clin Microbiol Infect Dis. 2013 ; 32: 1471-1481. 
333. Herpertz-Dahlmann B. Food matters: How the microbiome and gut-brain interaction might impact the development and course of anorexia nervosa. Eur Child Adolesc Psychiatry. 2017; 26: 1031-1041.

334. Mack I, Cuntz U, Grämer C, Niedermaier S, Pohl C, Schwiertz A, et al. Weight gain in anorexia nervosa does not ameliorate the faecal microbiota, branched chain fatty acid profiles, and gastrointestinal complaints. Sci Rep. 2016; 6: 26752.

335. Tennoune N, Chan P, Breton J, Legrand R, Chabane YN, Akkermann K, et al. Bacterial ClpB heatshock protein, an antigen-mimetic of the anorexigenic peptide alpha-MSH, at the origin of eating disorders. Transl Psychiatry. 2014; 4: e458.

336. Harach T, Marungruang N, Duthilleul N, Cheatham V, Bolmont T. Erratum: Reduction of Abeta amyloid pathology in APPPS1 transgenic mice in the absence of gut microbiota. Sci Rep. 2017; 7: 41802-41810.

337. Gubiani D, Fabbretti E, Cestnik B, Lavrač N, Urbančič T. Outlier based literature exploration for cross-domain linking of Alzheimer's disease and gut microbiota. Expert Syst Appl. 2017: 386396.

338. Harach T, Marungruang N, Duthilleul N, Cheatham V, Mc Coy KD, Frisoni G, et al. Reduction of $A \beta$ amyloid pathology in APPPS1 transgenic mice in the absence of gut microbiota. Sci Rep. 2017;7: 41802

339.Lakhan SE, Caro M, Hadzimichalis N. NMDA Receptor activity in neuropsychiatric disorders. Front Psychiatry. 2013; 4: 52-57.

340.Schwartz K, Boles BR. Microbial amyloids--functions and interactions within the host. Curr Opin Microbiol. 2013; 16: 93-99.

341.Cox PA, Davis DA, Mash DC, Metcalf JS, Banack SA. Dietary exposure to an environmental toxin triggers neurofibrillary tangles and amyloid deposits in the brain. Proc Biol Sci. 2016; 283: 20152397

342. Hu X, Wang T, Jin F. Alzheimer's disease and gut microbiota. Sci China. 2016: 1006-1026.

343.Jeffery IB, O'Toole PW. Diet-microbiota interactions and their implications for healthy living. Nutrients. 2013; 5: 234-252.

344. Solas M, Puerta E, Ramirez MJ. Treatment options in Alzheimers disease: The GABA Story. Curr Pharm Des. 2015; 21: 4960-4971.

345.Gerard HC, Dreses-Werrlingloer U, Wildt KS, Deka S, Oszust C, Balin BJ, et al. Chlamydophila (Chlamydia) pneumoniae in the Alzheimer's brain. Fems Immunol Med Microbiol. 2006: 355366.

346. Emery DC, Shoemark DK, Batstone TE, Waterfall CM, Coghill JA, Cerajewska TL, et al. 16S rRNA next generation sequencing analysis shows bacteria in Alzheimer's post-mortem brain. Front Aging Neuroence. 2017; 9: 195.

347.Vogt NM, Kerby RL, Dill-Mcfarland KA, Harding SJ, Merluzzi AP, Johnson SC, et al. Gut microbiome alterations in Alzheimer's disease. Sci Rep. 2017; 7: P563.

348. Kountouras J, Boziki M, Zavos C, Gavalas E, Vardaka E. A potential impact of chronic Helicobacter pylori infection on Alzheimer's disease pathobiology and course. Neurobiology of Aging. 2012; 33: e3-e4.

349. Pistollato F, Sumalla Cano S, Elio I, Masias Vergara M, Giampieri F, Battino M. Role of gut microbiota and nutrients in amyloid formation and pathogenesis of Alzheimer disease. Nutr Rev. 2016; 74: 624-634. 
350. Prandota J. Possible link between Toxoplasma gondii and the anosmia associated with neurodegenerative diseases. Am J Alzheimers Dis Other Demen. 2014; 29: 205-214.

351. Bonfili L, Cecarini V, Berardi S, Scarpona S, Suchodolski JS, Nasuti C, et al. Microbiota modulation counteracts Alzheimer's disease progression influencing neuronal proteolysis and gut hormones plasma levels. Sci Rep. 2017; 7: 2426.

352. Eskelinen MH, Ngandu T, Tuomilehto J, Soininen H, Kivipelto M. Midlife coffee and tea drinking and the risk of late-life dementia: A population-based CAIDE study. J Alzheimers Dis. 2009; 16: 85-91.

353. Cowan TE, PalmnoS MS, Yang J, Bomhof MR, Ardell KL, Reimer RA, et al. Chronic coffee consumption in the diet-induced obese rat: Impact on gut microbiota and serum metabolomics. J Nutr Biochem. 2014; 25: 489-495.

354. Mendiola-Precoma J, Berumen LC, Padilla K, Garcia-Alcocer G. Therapies for prevention and treatment of alzheimer's disease. BioMed Res Inter. 2016; 2016: 1-17.

355. Marta S, Katarzyna DL, Satler DB, Donata K, Ewa B, Jerzy L. The gut microbiome alterations and inflammation-driven pathogenesis of Alzheimer's disease - a critical review. Mol Neurobiol. 2018; 56: 1841-1851.

356. Dickson DW, Fujishiro H, Orr C, DelleDonne A, Josephs KA, Frigerio R, et al. Neuropathology of non-motor features of Parkinson disease. Parkinsonism Relat Disord. 2009; 15: S1-S3.

357. Holmqvist S, Chutna O, Bousset L, Aldrin-Kirk P, Li W, BjoRklund T, et al. Direct evidence of Parkinson pathology spread from the gastrointestinal tract to the brain in rats. Acta Neuropathol. 2014; 128: 805-820.

358.Scheperjans F, Aho V, Pereira PA, Koskinen K, Paulin L, Pekkonen E, et al. Gut microbiota are related to Parkinson's disease and clinical phenotype. Mov Disord. 2015; 30: 350-358.

359. Kelly LP, Carvey PM, Keshavarzian A, Shannon KM, Shaikh M, Bakay RA, et al. Progression of intestinal permeability changes and alpha-synuclein expression in a mouse model of Parkinson's disease. Mov Disord. 2013: 999-1009.

360.Tan AH, Mahadeva S, Thalha AM, Gibson PR, Kiew CK, Yeat CM, et al. Small intestinal bacterial overgrowth in Parkinson's disease. Parkinsonism Relat Disord. 2014; 2015: 535-540.

361. Chen WC, Quigley EM. Probiotics, prebiotics \& synbiotics in small intestinal bacterial overgrowth: Opening up a new therapeutic horizon! Indian J Med Res. 2014; 40: 582-584.

362. Keshavarzian A, Green SJ, Engen PA, Voigt RM, Naqib A, Forsyth CB, et al. Colonic bacterial composition in Parkinson's disease. Mov Disord. 2015; 30: 1351-1360.

363.Sara G, Mohajeri M. Changes of Colonic Bacterial Composition in Parkinson's Disease and Other Neurodegenerative Diseases. Nutrients. 2018; 10: 708-714.

364.Li W, Wu X, Hu X, Wang T, Liang S, Duan Y, et al. Structural changes of gut microbiota in Parkinson's disease and its correlation with clinical features. Sci China Life Sci. 2017; 60: 12231233.

365. Alvarez-Arellano L, Maldonado-Bernal C. Helicobacter pylori and neurological diseases: Married by the laws of inflammation. World J Gastrointest Pathophysiol. 2014; 4: 400-404.

366. Dobbs SM, Dobbs RJ, Weller C, Charlett A. Link between Helicobacter pylori infection and idiopathic parkinsonism. Med Hypotheses. 2000; 55: 93-98.

367. Blaecher C, Smet A, Flahou B, Pasmans F, Haesebrouck F. Significantly higher frequency of Helicobacter suis in patients with idiopathic parkinsonism than in control patients. Aliment Pharmacol Ther. 2013; 38: 1347-1353. 
368.Jang H, Boltz D, Sturm-Ramirez K, Shepherd KR, Yun J, Webster R, et al. Highly pathogenic H5N1 influenza virus can enter the central nervous system and induce neuroinflammation and neurodegeneration. Proc Natl Acad Sci U S A. 2009; 106, 14063-14068.

369. Wu WY, Kang KH, Chen SS, Chiu SH, Yen AF, Fann JY, et al. Hepatitis C virus infection: A risk factor for Parkinson's disease. J Viral Hepat. 2015; 22: 784-791.

370.Agata M. Brain-gut-microbiota axis in Parkinson's disease. World J Gastroenterol. 2015; 21: 10609-10620.

371. Reza TO, Mohsen T, Reza DK, Ebrahim K, Fereshteh B, Shokoofeh B, et al. Clinical and metabolic response to probiotic administration in people with Parkinson's disease: A randomized, doubleblind, placebo-controlled trial. Clin Nutrit. 2018; 38: 1031-1035.

372. Kirby TO, Ochoa-Repáraz J. The gut microbiome in multiple sclerosis: A potential therapeutic avenue. Med Sci. 2018; 6: 69-78.

373. Ochoa-Repáraz J, Mielcarz DW, Ditrio LE, Burroughs AR, Foureau DM, Haque-Begum S, et al. Role of gut commensal microflora in the development of experimental autoimmune encephalomyelitis. J Immunol. 2010; 183: 6041-6050.

374. Kirby T, Ochoa-Repáraz J. The gut microbiome in multiple sclerosis: A potential therapeutic avenue. Med Sci. 2018; 6: 69-78.

375.Probstel AK, Baranzini SE. The role of the gut microbiome in multiple sclerosis risk and progression: Towards characterization of the "MS Microbiome". Neurotherapeutics. 2018; 15: 126-134.

376. Tremlett $H$, Waubant E. The multiple sclerosis microbiome? Ann Transl Med. 2017; 5: 53-62.

377. Berer K, Gerdes LA, Cekanaviciute E, Jia X, Xiao L, Xia Z, et al. Gut microbiota from multiple sclerosis patients enables spontaneous autoimmune encephalomyelitis in mice. Proc Natl Acad Sci U S A. 2017; 114: 10719-10724.

378. Cekanaviciute E, Yoo BB, Runia TF, Debelius JW, Singh S, Nelson CA, et al. Gut bacteria from multiple sclerosis patients modulate human $T$ cells and exacerbate symptoms in mouse models. Proc Natl Acad Sci U S A. 2017; 114: 10713-10718.

379.Derrien M, Vaughan EE, Plugge CM, de Vos WM. Akkermansia muciniphila gen. nov., sp. nov., a human intestinal mucin-degrading bacterium. Int J Syst Evol Microbiol. 2004; 54: 1469-1476.

380. Freedman SN, Shahi SK, Mangalam AK. The "Gut Feeling": Breaking down the role of gut microbiome in multiple sclerosis. Neurotherapeutics. 2018; 15: 109-125.

381. Ochoa-Reparaz J, Mielcarz DW, Wang Y, Begum-Haque S, Dasgupta S, Kasper DL, et al. A polysaccharide from the human commensal Bacteroides fragilis protects against CNS demyelinating disease. Mucosal Immunol. 2010; 3: 487-495.

382.Yun KL, Menezes JS, Umesaki Y, Mazmanian SK. Proinflammatory T-cell responses to gut microbiota promote experimental autoimmune encephalomyelitis. Proc Natl Acad U S A. 2011; 108: 4615-4622.

383. Remot A, Descamps D, Noordine ML, Boukadiri A, Mathieu E, Robert V, et al. Bacteria isolated from lung modulate asthma susceptibility in mice. ISME J. 2017; 11: 1061-1074.

384. Hilty M, Burke C, Pedro H, Cardenas P, Bush A, Bossley C, et al. Disordered microbial communities in asthmatic airways. PloS one. 2012; 5: e8578.

385. Goleva E, Jackson LP, Harris JK, Robertson CE, Sutherland ER, Hall CF, et al. The effects of airway microbiome on corticosteroid responsiveness in asthma. Am J Respir Crit Care Med. 2013; 188: 1193-1201. 
386. Arrieta MC, Stiemsma LT, Dimitriu PA, Thorson L, Russell S, Yurist-Doutsch S, et al. Early infancy microbial and metabolic alterations affect risk of childhood asthma. Sci Transl Med. 2015; 7: 307 ra152.

387. Valdes AM, Jens W, Eran S, Spector TD. Role of the gut microbiota in nutrition and health. BMJ Clin Res. 2018; 361: k2179.

388.Schuijt TJ, Lankelma JM, Scicluna BP, Melo FD, Roelofs JJ, De Boer JD, et al. The gut microbiota plays a protective role in the host defence against pneumococcal pneumonia. Gut. 2016; 65: 575-583.

389. Charlson ES, Diamond JM, Bittinger K, Fitzgerald AS, Yadav A, Haas AR, et al. Lung-enriched organisms and aberrant bacterial and fungal respiratory microbiota after lung transplant. Am J Respir Crit Care Med. 2012; 186: 536-545.

390. Marsland BJ, Gollwitzer ES. Host-microorganism interactions in lung diseases. Nat Rev Immunol. 2014; 14: 827-835.

391. Kim SH, Clark ST, Surendra A, Copeland JK, Wang PW, Ammar R, et al. Global analysis of the fungal microbiome in cystic fibrosis patients peveals loss of function of the transcriptional Repressor Nrg1 as a Mechanism of Pathogen Adaptation. Plos Pathogens. 2015; 11: e1005308.

392. Wheeler ML, Limon JJ, Bar AS, Leal CA, Gargus M, Tang J, et al. Immunological consequences of intestinal fungal dysbiosis. Cell Host Microbe. 2016; 19: 865-873.

393. Marshall BJ, Warren JR. Unidentified curved bacilli in the stomach of patients with gastritis and peptic ulceration. Lancet. 1984; 1: 1311-1315.

394. Verma D, Garg PK, Dubey AK. Insights into the human oral microbiome. Arch Microbiol. 2018; 200: 525-540.

395.Zhao H, Chu M, Huang Z, Yang X, Ran S, Hu B, et al. Variations in oral microbiota associated with oral cancer. Sci Rep. 2017; 7: Art11773.

396.Sharma N, Bhatia S, Sodhi AS, Batra N. Oral microbiome and health. AIMS Microbiol. 2018; 4: 42-66.

397.Perera M, Al-Hebshi NN, Perera I, Ipe D, Ulett GC, Speicher DJ, et al. Inflammatory bacteriome and oral squamous cell carcinoma. J Dent Res. 2018; 97: 725-732.

398. Hsiao JR, Chang CC, Lee WT, Huang CC, Ou CY, Tsai ST, et al. The interplay between oral microbiome, lifestyle factors and genetic polymorphisms in the risk of oral squamous cell carcinoma. Carcinogenesis. 2018; 97: 778-787.

399. Takahashi Y, Park J, Hosomi K, Yamada T, Kobayashi A, Yamaguchi Y, et al. Analysis of oral microbiota in Japanese oral cancer patients using 16 S rRNA sequencing. J Oral Biosci. 2019; 61: 120-128.

400.Karpiński T. Role of oral microbiota in cancer development. Microorganisms. 2019; 7: 20-27.

401. Alnuaimi AD, Wiesenfeld D, O'Brien-Simpson NM, Reynolds EC, McCullough MJ. Oral candida colonization in oral cancer patients and its relationship with traditional risk factors of oral cancer: A matched case-control study. Oral Oncol. 2015; 51: 139-145.

402.Zhang Y, Wang X, Li H, Ni C, Yan F. Human oral microbiota and its modulation for oral health. Biomedicine \& pharmacotherapy = Biomedecine \& pharmacotherapie. 2018; 99: 883-893.

403. May M, Abrams JA. Emerging Insights into the Esophageal Microbiome. Curr Treat Options Gastroenterol. 2018; 16: 72-85.

404. Okereke I, Hamilton C, Wenholz A, Jala V, Giang T, Reynolds S, et al. Associations of the microbiome and esophageal disease. J Thorac Dis. 2019; 11: S1588-S1593. 
405. Osias GL, Bromer MQ, Thomas RM, Friedel D, Miller LS, Suh B, et al. Esophageal bacteria and Barrett's esophagus: A preliminary report. Dig Dis Ences. 2004; 49: 228-236.

406. Yang L, Lu X, Nossa CW, Francois F, Peek RM, Pei Z. Inflammation and intestinal metaplasia of the distal esophagus are associated with alterations in the microbiome. Gastroenterology. 2009; 137: 588-597.

407.Lv J, Guo L, Liu JJ, Zhao HP, Zhang J, Wang JH. Alteration of the esophageal microbiota in Barrett's esophagus and esophageal adenocarcinoma. World J Gastroenterol. 2019; 25: 21492161.

408. Narikiyo M, Tanabe C, Yamada Y, Igaki H, Sasaki H. Frequent and preferential infection of Treponema denticola, Streptococcus mitis, and Streptococcus anginosus in esophageal cancers. Cancer Sci. 2010; 95: 569-574.

409. Elliott DR, Walker AW, O'Donovan M, Parkhill J, Fitzgerald RC. A non-endoscopic device to sample the oesophageal microbiota: A case-control study. Lancet Gastroenterol Hepatol. 2017; 2: $32-42$.

410.Rubenstein JH, Shaheen NJ. Epidemiology, Diagnosis, and management of esophageal adenocarcinoma. Gastroenterology. 2015; 149: 302-317.

411. Nasrollahzadeh D, Malekzadeh R, Ploner A, Shakeri R, Sotoudeh M, Fahimi S, et al. Variations of gastric corpus microbiota are associated with early esophageal squamous cell carcinoma and squamous dysplasia. Sci Rep. 2015; 5: 8820-8828.

412.Gao S, Li S, Ma Z, Liang S, Shan T, Zhang M, et al. Presence of porphyromonas gingivalis in esophagus and its association with the clinicopathological characteristics and survival in patients with esophageal cancer. Infect Agent Cancer. 2016; 11: 3-11.

413. Chen X, Winckler B, Lu M, Cheng H, Yuan Z, Yang Y, et al. Oral microbiota and risk for esophageal squamous cell carcinoma in a high-risk area of China. PloS one. 2015; 10: e0143603.

414.Wang Q, Rao Y, Guo X, Liu N, Liu S, Wen P, et al. Oral microbiome in patients with oesophageal squamous cell carcinoma. Sci Rep. 2019; 9: 19055.

415. de Martel C, Ferlay J, Franceschi S, Vignat J, Bray F, Forman D, et al. Global burden of cancers attributable to infections in 2008: A review and synthetic analysis. Lancet Oncol. 2012; 13: 607615.

416.Iva P, Sofia J, Wichmann IA, Alejandra SB, Nicolás CV, Corvalán AH. Role of microRNAs and Exosomes in Helicobacter pylori and Epstein-Barr Virus Associated Gastric Cancers. Front Microbiol. 2018; 9: 636-642.

417.Schulz C, Schutte K, Mayerle J, Malfertheiner P. The role of the gastric bacterial microbiome in gastric cancer: Helicobacter pylori and beyond. Therap Adv Gastroenterol. 2019; 12: 1-11.

418.El-Omar EM, Carrington M, Chow WH, McColl KE, Bream JH, Young HA, et al. Interleukin-1 polymorphisms associated with increased risk of gastric cancer. Nature. 2000; 404: 398-402.

419. Herrera V, Parsonnet J. Helicobacter pylori and gastric adenocarcinoma. Clin Microbiol Infect. 2009; 15: 971-976.

420. Rhead JL, Letley DP, Mohammadi M, Hussein N, Mohagheghi MA, Hosseini ME, et al. A new helicobacter pylori vacuolating cytotoxin determinant, the intermediate region, is associated with gastric cancer. Gastroenterology. 2007; 133: 926-936.

421.Oertli M, Sundquist M, Hitzler I, Engler DB, Arnold IC, Reuter S, et al. DC-derived IL-18 drives Treg differentiation, murine Helicobacter pylori-specific immune tolerance, and asthma protection. J Clin Invest. 2012; 122: 1082-1096. 
422. Blaser MJ, Perez-Perez GI, Kleanthous H, Cover TL, Peek RM, Chyou PH, et al. . Infection with Helicobacter pylori Strains Possessing cagA Is Associated with an Increased Risk of Developing Adenocarcinoma of the Stomach. Cancer Res. 1995; 55: 2111-2115.

423. Kaparakis M, Turnbull L, Carneiro L, Firth S, Coleman HA, Parkington HC, et al. Bacterial membrane vesicles deliver peptidoglycan to NOD1 in epithelial cells. Cell Microbiol. 2010; 12: 372-385.

424. Nardone G, Compare D. The human gastric microbiota: Is it time to rethink the pathogenesis of stomach diseases? Un Eur Gastroenterol J. 2015; 9: 255-260.

425. Atherton JC, Blaser MJ. Coadaptation of Helicobacter pylori and humans: Ancient history, modern implications. J Clin Invest. 2009; 119: 2475-2487.

426. Blaser MJ, Atherton JC. Helicobacter pylori persistence: Biology and disease. J Clin Invest. 2004; 113: 321-333.

427.Gantuya B, El-Serag HB, Matsumoto T, Ajami NJ, Oyuntsetseg K, Azzaya D, et al. Gastric microbiota in Helicobacter pylori-negative and -positive gastritis among high incidence of gastric cancer area. Cancers. 2019; 11: 504-511.

428. Erawijantari PP, Mizutani S, Shiroma H, Shiba S, Nakajima T, Sakamoto T, et al. Influence of gastrectomy for gastric cancer treatment on fecal microbiome and metabolic profiles. Gut. 2020; 0: 1-12.

429. Chen XH, Wang A, Chu AN, Gong YH, Yuan Y. Mucosa-associated microbiota in gastric cancer tissues compared with non-cancer tissues. Front Microbiol. 2019; 10: Art1261.

430. Ferreira RM, Pereira-Marques J, Pinto-Ribeiro I, Costa JL, Carneiro F, Machado JC, et al. Gastric microbial community profiling reveals a dysbiotic cancer-associated microbiota. Gut. 2017: 226-236.

431. Coker OO, Dai Z, Nie Y, Zhao G, Yu J. Mucosal microbiome dysbiosis in gastric carcinogenesis. Gut. 2018; 67: 1024-1032.

432.Lee YC, Chiang TH, Chou CK, Tu YK, Liao WC, Wu MS, et al. Association between helicobacter pylori eradication and gastric cancer incidence: A systematic review and meta-analysis. Gastroenterology. 2016; 150: 1113-1124.

433. Shiryaev SA, Remacle AG, Chernov AV, Golubkov VS, Motamedchaboki K, Muranaka N, et al. . Substrate cleavage profiling suggests a distinct function of Bacteroides metalloproteinases (fragilysin and metalloproteinase II) at the microbiome-inflammation-cancer interface. J Biol Chem. 2013; 288: 34956-34967.

434.Wu S, Rhee KJ, Albesiano E, Rabizadeh S, Wu X, Yen HR, et al. A human colonic commensal promotes colon tumorigenesis via activation of $\mathrm{T}$ helper type $17 \mathrm{~T}$ cell responses. Nat Med. 2009; 15: 1016-1022.

435. Wang B, Yao M, Lv L, Ling Z, Li L. The human microbiota in health and disease. Engineering. 2017; 3: 71-82.

436.Davis CD, Milner JA. Gastrointestinal microflora, food components and colon cancer prevention. J Nutr Biochem. 2009; 20: 743-752.

437. Sobhani I, Bergsten E, Couffin S, Amiot A, Nebbad B, Barau C, et al. Colorectal cancer-associated microbiota contributes to oncogenic epigenetic signatures. Proc Natl Acad Sci U S A. 2019; 116: 24285-24295.

438.Iradj S, Julien T, FranOise RT, Roperch JP, Sophie L, Philippe L, et al. Microbial dysbiosis in colorectal cancer (CRC) Patients. PloS one. 2011; 6: e16393. 
439. Wang T, Cai G, Qiu Y, Fei N, Zhang M, Pang X, et al. Structural segregation of gut microbiota between colorectal cancer patients and healthy volunteers. ISME J. 2012; 6: 320-329.

440.Sanapareddy N, Legge RM, Jovov B, McCoy A, Burcal L, Araujo-Perez F, et al. Increased rectal microbial richness is associated with the presence of colorectal adenomas in humans. ISME J. 2012; 6: 1858-1868.

441.Saus E, Iraola-Guzman S, Willis JR, Brunet-Vega A, Gabaldon T. Microbiome and colorectal cancer: Roles in carcinogenesis and clinical potential. Mol Aspects Med. 2019; 69: 93-106.

442. Thomas AM, Manghi P, Asnicar F, Pasolli E, Armanini F, Zolfo M, et al. Metagenomic analysis of colorectal cancer datasets identifies cross-cohort microbial diagnostic signatures and a link with choline degradation. Nat Med. 2019; 25: 667-678.

443. Wirbel J, Pyl PT, Kartal E, Zych K, Kashani A, Milanese A, et al. Meta-analysis of fecal metagenomes reveals global microbial signatures that are specific for colorectal cancer. Nat Med. 2019; 25: 679-689.

444. Moore WE, Moore LH. Intestinal floras of populations that have a high risk of colon cancer. Appl Environ Microbiol. 1995; 61: 3202-3207.

445. O'Keefe SJ, Chung D, Mahmound N, Sepulveda AR, Manafe M, Arch J, et al. Why do African Americans get more colon cancer than Native Africans? J Nutr. 2007; 137: 175S-182S.

446. Kostic AD, Chun E, Robertson L, Glickman JN, Gallini CA, Michaud M, et al. Fusobacterium nucleatum potentiates intestinal tumorigenesis and modulates the tumor-immune microenvironment. Cell Host Microbe. 2013; 14: 207-215.

447. Blake SJ, Dougall WC, Miles JJ, Teng MW, Smyth MJ. Molecular Pathways: Targeting CD96 and TIGIT for Cancer Immunotherapy. Clin Cancer Res. 2016; 22: 5183-5188.

448. Yan X, Liu L, Li H, Qin H, Sun Z. Clinical significance of Fusobacterium nucleatum, epithelialmesenchymal transition, and cancer stem cell markers in stage III/IV colorectal cancer patients. Oncotarget Ther. 2017; 10: 5031-5046.

449.Goodwin AC, Shields CE, Wu S, Huso DL, Wu X, Murray-Stewart TR, et al. Polyamine catabolism contributes to enterotoxigenic Bacteroides fragilis-induced colon tumorigenesis. Proc Natl Acad Sci U S A. 2011; 108: 15354-15359.

450.Geis AL, Fan H, Wu X, Wu S, Huso DL, Wolfe JL, et al. Regulatory T-cell response to enterotoxigenic Bacteroides fragilis colonization triggers IL17-dependent colon carcinogenesis. Cancer Discov. 2015; 5: 1098-1109.

451. Arthur JC, Perez-Chanona E, Muhlbauer M, Tomkovich S, Uronis JM, Fan TJ, et al. Intestinal inflammation targets cancer-inducing activity of the microbiota. Science. 2012; 338: 120-123.

452. Wilson MR, Jiang Y, Villalta PW, Stornetta A, Boudreau PD, Carra A, et al. The human gut bacterial genotoxin colibactin alkylates DNA. Science. 2019; 363: eaar7785.

453. Cougnoux A, Dalmasso G, Martinez R, Buc E, Delmas J, Gibold L, et al. Bacterial genotoxin colibactin promotes colon tumour growth by inducing a senescence-associated secretory phenotype. Gut. 2014; 63: 1932-1942.

454.Wang C, Li J. Pathogenic Microorganisms and Pancreatic Cancer. Gastrointest Tumors. 2015; 2: 41-47.

455. Maekawa T, Fukaya R, Takamatsu S, Itoyama S, Fukuoka T, Yamada M, et al. Possible involvement of Enterococcus infection in the pathogenesis of chronic pancreatitis and cancer. Biochem Biophys Res Commun. 2018; 506: 962-969.

456. Dickson I. Microbiome promotes pancreatic cancer. Nat Rev Dis Primers. 2018; 15: 328-332. 
457. Mitsuhashi K, Nosho K, Sukawa Y, Matsunaga Y, Ito M, Kurihara H,et al. Association of Fusobacterium species in pancreatic cancer tissues with molecular features and prognosis. Oncotarget. 2015; 6: 7209-7222.

458.Geller LT, Barzili-Rokni M, Danino T, Jonas OH, Shental N, Nejman D, et al. Potential role of intratumor bacteria in mediating tumor resistance to the chemotherapeutic drug gemcitabine. Science. 2017; 357: 1156-1160.

459. Wei MY, Shi S, Liang C, Meng QC, Hua J, Zhang YY, et al. The microbiota and microbiome in pancreatic cancer: More influential than expected. Mol Cancer. 2019; 18: 97.

460. Di Magliano MP, Logsdon CD. Roles for KRAS in pancreatic tumor development and progression. Gastroenterology. 2013; 144: 1220-1229.

461. Fukuda A, Wang SC, Morris IV JP, Folias AE, Liou A, Kim GE, et al. Stat3 and MMP7 contribute to pancreatic ductal adenocarcinoma initiation and progression. Cancer Cell. 2011; 19: 441-455.

462. Gaiser RA, Halimi A, Alkharaan H, Lu L, Davanian H, Healy K, et al. Enrichment of oral microbiota in early cystic precursors to invasive pancreatic cancer. Gut. 2019; 68: 2186-2194.

463. Michaud DS, Izard J, Wilhelm-Benartzi CS, You DH, Grote VA, Tjonneland A, et al. Plasma antibodies to oral bacteria and risk of pancreatic cancer in a large European prospective cohort study. Gut. 2013; 62: 1764-1770.

464. Farrell JJ, Zhang L, Zhou H, Chia D, Elashoff D, Akin D, et al. Variations of oral microbiota are associated with pancreatic diseases including pancreatic cancer. Gut. 2012; 61: 582-588.

465. Fan X, Alekseyenko AV, Wu J, Peters BA, Jacobs EJ, Gapstur SM, et al. Human oral microbiome and prospective risk for pancreatic cancer: A population-based nested case-control study. Gut. 2018; 67: 120-127.

466. Ogrendik M. Periodontal pathogens in the etiology of pancreatic cancer. Gastrointest Tumors. 2017; 3: 125-127.

467. Half E, Keren N, Dorfman T, Reshef L, Lachter I, Kluger Y, et al. P-165Specific changes in fecal microbiota may differentiate pancreatic cancer patients from healthy individuals. Ann Oncol. 2015; 26: iv48.

468. Sethi V, Vitiello GA, Saxena D, Miller G, Dudeja V. The role of the microbiome in immunologic development and its implication for pancreatic cancer immunotherapy. Gastroenterology. 2019; 156: 2097-2115 e2092.

469. Riquelme E, Zhang Y, Zhang L, Montiel M, McAllister F. Tumor microbiome diversity and composition influence pancreatic cancer outcomes. Cell. 2019; 178: 795-806.

470.Vitiello GA, Cohen DJ, Miller G. Harnessing the Microbiome for Pancreatic Cancer Immunotherapy. Trends Cancer. 2019; 5: 670-676.

471.Ponziani FR, Gerardi V, Pecere S, D'Aversa F, Lopetuso L, Zocco MA, et al. Effect of rifaximin on gut microbiota composition in advanced liver disease and its complications. World J Gastroenterol. 2015; 21: 12322-12333.

472.Grat M, Wronka KM, Krasnodebski M, Masior L, Lewandowski Z, Kosinska I, et al. Profile of gut microbiota associated with the presence of hepatocellular cancer in patients with liver cirrhosis. Transplant Proc. 2016; 48: 1687-1691.

473.Ponziani FR, Bhoori S, Castelli C, Putignani L, Rivoltini L, Del Chierico F, et al. Hepatocellular carcinoma is associated with gut microbiota profile and inflammation in nonalcoholic fatty fiver disease. Hepatology. 2019; 69: 107-120. 
474. Ren Z, Li A, Jiang J, Lin Z, Yu Z, Lu H, et al. Gut microbiome analysis as a tool towards targeted non-invasive biomarkers for early hepatocellular carcinoma. Gut. 2018; 68: 1014-1023.

475.Li N. Identification of helicobacter species in human liver samples from patients with primary hepatocellular carcinoma. J Clin Pathol. 2004; 57: 1273-1277.

476.Dore MP, Realdi G, Mura D, Graham DY, Sepulveda AR. Helicobacter infection in patients with HCV-Related chronic hepatitis, cirrhosis, and hepatocellular carcinoma. Dig Dis. 2002; 47: 16381643.

477.Zhou D, Wang JD, Weng MZ, Zhang Y, Wang XF, Gong W, et al. Infections of Helicobacter spp. in the biliary system are associated with biliary tract cancer: A meta-analysis. Eur J Gastroenterol Hepatol. 2013; 25: 447-454.

478.Samaras V, Rafailidis PI, Mourtzoukou EG, Peppas G, Falagas ME. Chronic bacterial and parasitic infections and cancer: A review. J Infect Dev Ctries. 2010; 4: 267-281.

479.Jiang JW, Chen XH, Ren Z, Zheng SS. Gut microbial dysbiosis associates hepatocellular carcinoma via the gut-liver axis. Hepatobiliary Pancreat Dis Int. 2019; 18: 19-27.

480. Loo TM, Kamachi F, Watanabe Y, Yoshimoto S, Kanda H, Arai Y, et al. Gut microbiota promotes obesity-associated liver cancer through PGE2-Mediated suppression of antitumor immunity. Cancer Discov. 2017; 7: 522-538.

481. Hartmann P, Chu H, Duan Y, Schnabl B. Gut microbiota in liver disease: Too much is harmful, nothing at all is not helpful either. Am J Physiol Gastrointest Liver Physiol. 2019; 316: G563G573.

482. Wang L, Wan YJ. The role of gut microbiota in liver disease development and treatment. Liver Res. 2019; 3: 3-18

483. Kovaleva OV, Romashin D, Zborovskaya IB, Davydov MM, Shogenov MS, Gratchev A. Human Lung microbiome on the way to cancer. J Immunol Res. 2019; 2019: Art ID 1394191.

484. Mrázek J, Mekadim C, Kučerova P, Švejstil R, Salmonová H, Vlasáková J, et al. Melanoma-related changes in skin microbiome. Folia Microbiol. 2019; 64: 435-442

485. Farolfi A, Gurioli G, Fugazzola P, Burgio SL, Giorgi UD. Immune System and DNA repair defects in ovarian cancer: Implications for locoregional approaches. Int J Mol Sci. 2019; 20: 2569-2577. 486.Siegel RL, Miller KD, Jemal A. Cancer statistics, 2019. CA Cancer J Clin. 2019; 69: 7-34.

487. Mikó E, Kovács T, Sebő É, Tóth J, Csonka T, Ujlaki G, et al. Microbiome-Microbial MetabolomeCancer cell interactions in breast cancer - Familiar, but unexplored. Cells. 2019; 8: 293-297.

488. Maslowski KM, Vieira AT, Ng A, Kranich J, Sierro F, Yu D, et al. Regulation of inflammatory responses by gut microbiota and chemoattractant receptor GPR43. Nature. 2009; 461: 12821286. 


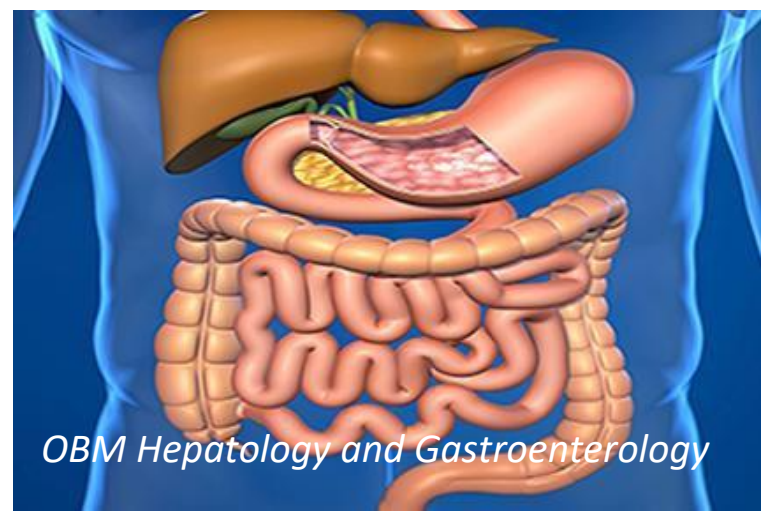

Enjoy OBM Hepatology and Gastroenterology by:

1. Submitting a manuscript

2. Joining in volunteer reviewer bank

3. Joining Editorial Board

4. Guest editing a special issue

For more details, please visit:

http://www.lidsen.com/journals/hg 